\title{
Measurement of $\mathrm{BR}(\mathrm{Bu}$ to phi $\mathrm{K}) / \mathrm{BR}(\mathrm{Bu}$ to $\mathrm{J} / \mathrm{psi} \mathrm{K})$ at the Collider Detector at Fermilab
}

\author{
Robert A. Napora \\ A dissertation submitted to the Johns Hopkins University in conformity with the \\ requirements for the degree of Doctor of Philosophy. \\ Baltimore, Maryland \\ 2004
}




\begin{abstract}
This thesis presents evidence for the decay mode $B^{ \pm} \rightarrow \phi K^{ \pm}$in $p \bar{p}$ collisions at $\sqrt{s}=1.96 \mathrm{TeV}$ using $(120 \pm 7) \mathrm{pb}^{-1}$ of data collected by the Collider Detector at Fermilab (CDF). This signal is then used to measure the branching ratio relative to the decay mode $B^{ \pm} \rightarrow J / \psi K^{ \pm}$.

The measurement starts from reconstructing the two decay modes:

$$
B^{ \pm} \rightarrow \phi K^{ \pm}, \text {where } \phi \rightarrow K^{+} K^{-}
$$

and

$$
B^{ \pm} \rightarrow J / \psi K^{ \pm}, \text {where } J / \psi \rightarrow \mu^{+} \mu^{-}
$$

The measurement yielded $23 \pm 7 B^{ \pm} \rightarrow \phi K^{ \pm}$events, and $406 \pm 26 B^{ \pm} \rightarrow J / \psi K^{ \pm}$ events. The fraction of $B^{ \pm} \rightarrow J / \psi K^{ \pm}$events where the $J / \psi$ subsequently decayed to two muons (as opposed to two electrons) was found to be $f_{\mu \mu}=0.839 \pm 0.066$. The relative branching ratio of the two decays is then calculated based on the equation:

$$
\frac{B R\left(B^{ \pm} \rightarrow \phi K^{ \pm}\right)}{B R\left(B^{ \pm} \rightarrow J / \psi K^{ \pm}\right)}=\frac{N_{\phi K}}{N_{\psi K} \cdot f_{\mu \mu}} \frac{B R\left(J / \psi \rightarrow \mu^{+} \mu^{-}\right)}{B R\left(\phi \rightarrow K^{+} K^{-}\right)} \frac{\epsilon_{\mu \mu K}}{\epsilon K K K} R\left(\epsilon_{i s o}\right)
$$
\end{abstract}

The measurement finds

$$
\frac{B R\left(B^{ \pm} \rightarrow \phi K^{ \pm}\right)}{B R\left(B^{ \pm} \rightarrow J / \psi K^{ \pm}\right)}=0.0068 \pm 0.0021 \text { (stat.) } \pm 0.0007 \text { (syst.) }
$$

The $B^{ \pm} \rightarrow \phi K^{ \pm}$branching ratio is then found to be

$$
B R\left(B^{ \pm} \rightarrow \phi K^{ \pm}\right)=[6.9 \pm 2.1(\text { stat. }) \pm 0.8(\text { syst. })] \times 10^{-6}
$$

This value is consistent with similar measurements reported by the $e^{+} e^{-}$collider experiments BaBar[1], Belle[2], and CLEO[3].

Adviser: Dr. Barry Blumenfeld 


\section{Acknowledgements}

First and foremost, I would like to thank my parents. Their unconditional love, support, and encouragement through the years have been the root of all of my accomplishments.

I owe the greatest debt of gratitude to my adviser, Prof. Barry Blumenfeld. I am thankful for having had the opportunity to work with him. His instruction, guidance, and support through the last six years have been invaluable. I am also very grateful for the freedom he has given me while under his tutelage, allowing me to pursue many of my own projects and interests. I could not have asked for a better adviser.

I would also like to thank the rest of the Johns Hopkins faculty. It was a privilege to study under so many great professors. In particular, I would like to thank Prof. Petar Maksimovic whose constant efforts truly made this analysis possible, and Prof. Bruce Barnett for his sage advice over the years. I would also like to thank Prof. Morris Swartz, Prof. C.Y. Chien, and the professor's from the high energy theory group. Thanks to the students and staff at Johns Hopkins for providing support of a different kind. I have missed the entire department dearly since moving to Fermilab. I would particularly like to thank Janet Krupsaw for taking such great care of all us graduate students during her tenure as Academic Affairs Administrator.

Thanks to the Johns Hopkins group members who joined me at CDF. It was a pleasure working with all of you. Chadd Smith for showing me the ropes back in the early days. Le Yi for his frequent advice, and for being one of the best roommates I ever had. Yi Kai for being such a great study partner for the oral exams. Reid Mumford for his constant enthusiasm and his tireless efforts while working with me "down in the trenches" of the CDF collision hall. I would like to thank Dr.'s Satyajit Behari and Mat Martin for their input on this analysis. Most especially, I would like to thank Dr. Matt Herndon for answering my countless questions over the years and teaching me so very much.

This thesis would not have been possible without the efforts of all the members of CDF who spent countless hours designing, building, and operating the CDF detector. The entire Run II author list belongs here, but I would particularly like to thank 
Marco Rescigno, Saverio D'Auria, Massimo Casarsa, and rest of the Italian B group who did so much to make this thesis possible. I would also like to thank the B group leaders, Marjorie Shapiro and Christoph Paus, for their guidance and support, Steve Worm for our many useful conversations, and all the members of the silicon group who I spent so much time with: Jean Slaughter, Gino Bolla, Andy Foland, Steve Nahn, Lester Miller, Doug Glenzinski, David Stuart and so many more. I learned volumes working with all of you.

Finally, I would like to thank Kristin for her patience, love and support during the crazy times. 


\section{Contents}

List of Figures vii

List of Tables $\quad$ x

1 Introduction $\quad 1$

1.1 Historical Background . . . . . . . . . . . . . . . 1

1.2 High Energy Physics . . . . . . . . . . . . . . 3

1.3 Outline of this Thesis . . . . . . . . . . . . . . 4

2 Theoretical Motivation 5

2.1 The Standard Model of Particle Physics . . . . . . . . . . . 5

2.1.1 The Fundamental Fermions ............ 5

2.1 .2 Interactions . . . . . . . . . . . . . . . . . . 6

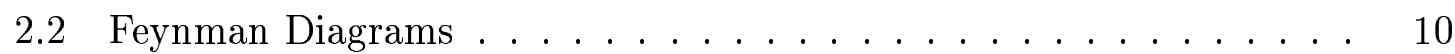

2.3 Weak Interactions and the CKM Matrix ............ 12

2.4 CP Violation ........................ 15

3 The Experimental Setup 21

3.1 The Tevatron ......................... 21

3.2 Collider Detector at Fermilab (CDF) . . . . . . . . . . . 23

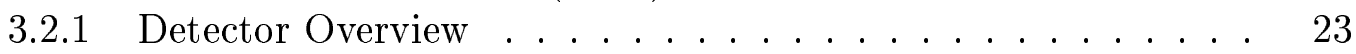

3.3 The Tracking System . . . . . . . . . . . . . . . . . . 25

3.3.1 Silicon Tracking . . . . . . . . . . . . . . 26

3.3 .2 Central Outer Tracker (COT) . . . . . . . . . . . . 30

3.4 Calorimeter Systems . . . . . . . . . . . . . . 31

3.5 Muon Detectors . . . . . . . . . . . . . . 33

3.6 Trigger Systems . . . . . . . . . . . . . . . . . . . . . . . . . . . . . . . . 35

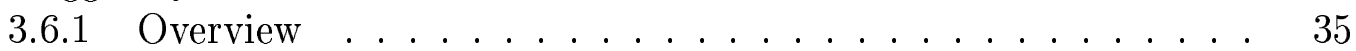

3.6.2 Level-1 Trigger . . . . . . . . . . . . . . 35

3.6.3 Level-2 Trigger . . . . . . . . . . . . . . . . . . . 38

3.6.4 Event Builder and Level-3 Trigger . . . . . . . . . . . 43 
4 Data Sample 44

4.1 Hadronic Data Sample . . . . . . . . . . . . . . . . . 44

4.1.1 The Nominal Two-Track Trigger Stream . . . . . . . . . . 45

4.1.2 The Low- $p_{T}$ Two-Track Trigger Stream . . . . . . . . . . . . . . . . 46

4.1.3 The High- $p_{T}$ Two-Track Trigger Stream . . . . . . . . . . . . 48

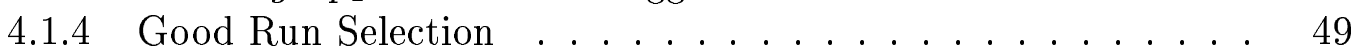

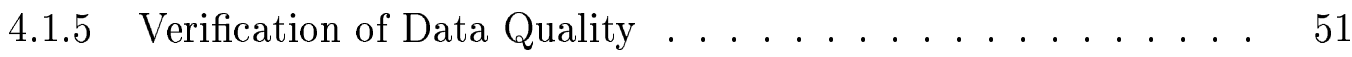

4.2 Monte Carlo Data Sample . . . . . . . . . . . . . 52

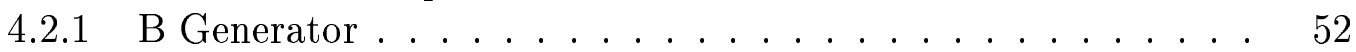

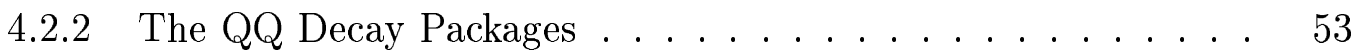

4.2.3 Trigger and Detector Simulation . . . . . . . . . 53

4.3 Offline Track Reconstruction and Refitting . . . . . . . . . . . 54

4.3.1 Offline Track Reconstruction ............. 54

4.3.2 Track Refitting .............. 56

5 Physics Analysis $\quad 58$

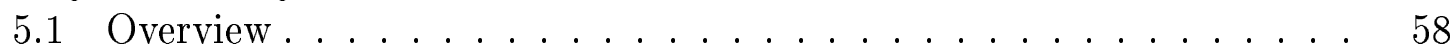

5.2 Candidate Selection . . . . . . . . . . . . . . . . 59

5.2.1 Track Quality Cuts ............... 59

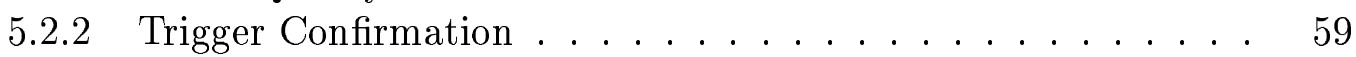

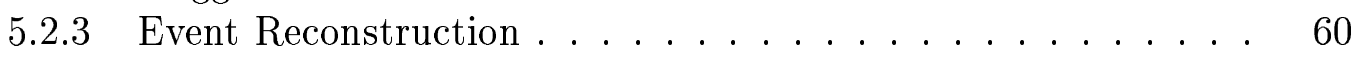

5.2.4 $B^{ \pm} \rightarrow \phi K^{ \pm}$Candidate Selection . . . . . . . . . . 62

5.3 The $B^{ \pm} \rightarrow J / \psi K^{ \pm}$Normalization Mode . . . . . . . . . . . 65

5.4 Relative Efficiencies from Monte Carlo Simulation . . . . . . . . . 68

5.5 Finding the Fraction of $J / \psi \rightarrow \mu^{+} \mu^{-}$Events in the $B^{ \pm} \rightarrow J / \psi K^{ \pm}$Signal 73

5.5.1 Fraction of $J / \psi \rightarrow \mu^{+} \mu^{-}$From the 4-Dimensional Fit . . . . 73

5.5.2 Fraction of $J / \psi \rightarrow \mu^{+} \mu^{-}$From the 2-Dimensional Fit . . . . 85

5.5.3 Fraction of $J / \psi \rightarrow \mu^{+} \mu^{-}$From the Monte Carlo . . . . . . 85

5.5.4 Fraction of $J / \psi \rightarrow \mu^{+} \mu^{-}$From Muon Chamber Identification . 87

5.5.5 Final Result for $J / \psi \rightarrow \mu^{+} \mu^{-}$Fraction . . . . . . . . . 89

6 Evaluation of Systematic Errors $\quad 90$

6.1 Width Constraint on $B \rightarrow \phi K$ Fit . . . . . . . . . . . . . . 90

6.2 Narrow Opening Angle of the $\phi \ldots \ldots . \ldots . \ldots . \ldots 92$

6.3 Isolation Cut Efficiency . . . . . . . . . . . . . . . . . . . 94

$6.4 \chi_{x y}^{2}$ Cut Efficiency . . . . . . . . . . . . . . . . 95

6.5 Estimated Muon Fraction in $B^{ \pm} \rightarrow J / \psi K^{ \pm} \ldots \ldots$. . . . . . 96

$\begin{array}{lll}7 & \text { Summary and Conclusions } & 100\end{array}$

$\begin{array}{ll}\text { Bibliography } & 104\end{array}$ 


\section{List of Figures}

2.1 Feynman diagram representing the scattering interaction of two electrons via photon exchange. . . . . . . . . . . . . . .

2.2 Sum of Feynman diagrams representing the electromagnetic scattering interaction between two electrons. . . . . . . . . . . . . . . .

2.3 Vertex showing an ingoing up quark with an outgoing $W^{+}$and outgoing quark $q$, where $q=d, s$, or $b$. This vertex must be multiplied by the element $V_{u q}$ of the CKM matrix describing the overlap of the emitted quark with the weak state partnered with the $u \ldots \ldots \ldots \ldots$. . .

2.4 A schematic representation of the interference responsible for CP violation. The strong phase angles are represented by $\delta_{i}$, and the weak phase angles are represented by $\phi_{i}$, for each amplitude, $A_{i}$. The left diagram shows the complex sum for the two amplitudes, $A_{1}+A_{2}$. The right diagram shows the complex sum of the CP conjugate amplitudes, $\bar{A}_{1}+\bar{A}_{2}$. Notice that while $\left|A_{1}\right|=\left|\bar{A}_{1}\right|$ and $\left|A_{2}\right|=\left|\bar{A}_{2}\right|$, the total amplitudes are different for the two cases, i.e. $\left|A_{1}+A_{2}\right| \neq\left|\bar{A}_{1}+\bar{A}_{2}\right|$. .

2.5 The Feynman Diagram for $b \rightarrow s \bar{s} s$. This actually represents three different amplitudes, since different quarks may participate in the internal lines of the loop; $q=u, c$, or $t . \ldots \ldots \ldots \ldots$

3.1 Diagram of the Fermilab accelerator chain. . . . . . . . . . . . 22

3.2 Cross-sectional view of the CDF detector. . . . . . . . . . . .

3.3 Cross-sectional view of the CDF tracking system for Run II, along with the plug calorimeter systems. . . . . . . . . . . . . 27

3.4 End view of the L00 tracker, shown surrounded by the first two layers of the SVX II. . . . . . . . . . . . . . . . . . . . 27

3.5 Diagram showing the three barrel structure of the SVX II. . . . . . . 29

3.6 Left: End view of the CDF Run II silicon system; Right: Side view of half of the CDF Run II silicon tracking system. Note that the scale of the z-coordinate has been highly compressed in this figure. . . . . . .

3.7 Nominal cell layout for superlayer 2, and arrangement of cells on the COT endplate. . . . . . . . . . . . . . . . . . 
3.8 A lateral view of one quarter of the CDF detector showing the orientation of the calorimeter and central muon systems. . . . . . . . . .

3.9 Geometry of muon counters in the central region. From left-to-right the muon passes through the absorber steel, the drift chamber cells, and the scintillators. . . . . . . . . . . . . .

3.10 Coverage of the various muon detectors in $\eta$ and $\phi . \ldots \ldots \ldots . . . .34$

3.11 A functional diagram of the CDF run-II trigger system. . . . . . . . 36

3.12 A block diagram of the CDF run-II trigger system. . . . . . . . . . 39

3.13 Architecture of the SVT trigger. . . . . . . . . . . . . 42

4.1 Plot of $D_{s} / D^{ \pm}$yield vs. run ranges. $\ldots \ldots \ldots \ldots \ldots \ldots$

5.1 Decay topologies for the decays considered in this analysis. Left: $B^{ \pm} \rightarrow$ $\phi K^{ \pm}$with the subsequent decay $\phi \rightarrow K^{+} K^{-}$. Right: $B^{ \pm} \rightarrow J / \psi K^{ \pm}$ with the subsequent decay $J / \psi \rightarrow l^{+} l^{-}$, where $l=(\mu, e) \ldots \ldots$. .

5.2 Left: $M_{K K}$ invariant mass for vertices in the B mass region Right: Zoomed in view of the left plot; red line: $\phi$ signal; cyan line: combinatorial background; green line: $K_{s}$ reflection. . . . . . . . . . . .

5.3 Left: Pt of the kaon not belonging to the trigger pair. Open histogram: data from $M_{\phi K}$ sidebands; Hatched histogram: $B^{ \pm} \rightarrow \phi K^{ \pm}$Monte Carlo. Right: Impact parameter for kaon not belonging to the trigger pair. Open histogram: data from $M_{\phi K}$ sidebands; Dashed histogram: $B^{ \pm} \rightarrow \phi K^{ \pm}$Monte Carlo . . . . . . . . . . . . . . . . 63

5.4 Mass plot of $B \rightarrow \phi K$ candidate events. . . . . . . . . . 64

5.5 Mass plot of $B \rightarrow J / \psi K$ events. . . . . . . . . . . . 66

5.6 Mass plot of $B \rightarrow J / \psi K$ from realistic Monte Carlo containing the decays $B_{0}, B_{s}, B^{ \pm}, \Lambda_{b} \rightarrow J / \psi+X \ldots \ldots \ldots \ldots$. . . . . . 67

5.7 The $B^{ \pm} \rightarrow J / \psi K^{ \pm}$Pt spectrum in Monte Carlo and data. . . . . . 68

5.8 Ratio of $\epsilon_{\phi K} / \epsilon_{\psi K}$ for the eight run numbers used in simulation. $\quad \ldots \quad 69$

5.9 The $d E / d x$ pull PDF for positrons. . . . . . . . . . . . 77

5.10 The $\mathrm{dE} / \mathrm{dx}$ pull PDFs for all particles calculated under a pion hypothesis. Yellow: protons, Magenta: kaons, Black: pions, Red: muons, Green: electrons. The blue curve is the sum over all particles. . . . .

5.11 Histogram of $B_{\text {mass }}$ vs. $\mathrm{J} / \psi_{\text {mass }}$. Note the bremsstrahlung tail in the direction of decreasing $\mathrm{B}$ and $J / \psi$ masses, at a $45^{\circ}$ angle to both axes.

5.12 Plot of the $J / \psi$ mass in data. Red: PDF for $J / \Psi \rightarrow \mu \mu$ (two Gaussians), Green: PDF for $J / \Psi \rightarrow e e$ (a Gaussian and a bifurcated Gaus$\operatorname{sian}) . \ldots \ldots \ldots \ldots \ldots \ldots \ldots \ldots$

5.13 Plot of the $B$ mass in data. Red: PDF for $B \rightarrow J / \Psi K \rightarrow \mu \mu K$ (a Gaussian), Green: PDF for $B \rightarrow J / \Psi K \rightarrow e e K$ (1-d projection of six 2-d Gaussians) $\ldots \ldots \ldots \ldots \ldots \ldots \ldots$ 
5.14 Left: $d E / d x$ pull projection for negatively charged particles. Right: $d E / d x$ pull projection for positively charged particles. . . . . . . .

5.15 Projection of the 4-dimensional fit on the B mass dimension after applying the analysis cuts on the $J / \psi$ mass. . . . . . . . . 84

$5.16|\Delta x|$ and $|\Delta \phi|$ distributions in data and Monte Carlo. . . . . . . 88

6.1 Left: $D_{s}$ and $D^{+}$mass distribution in data in the $\phi \pi$ decay mode. Right: $D_{s} \rightarrow \phi \pi$ Monte Carlo mass distribution . . . . . . . .

6.2 Number of events vs. opening angle of phi in $D_{s} \rightarrow \phi \pi$ events. The red points (red histogram) are for the data (Monte Carlo data) of the subset of events for which both the phi tracks participated in the trigger. MC: $2878 \phi$-triggers, Data: $2716 \phi$-triggers. . . . . . . . . .

6.3 Transverse momentum spectra of the selected candidates from the Monte Carlo sample for the two decay channels. . . . . . . . . .

6.4 Efficiency of the isolation cut at 0.5 , as measured with the $B^{ \pm} \rightarrow$ $J / \psi K^{ \pm}$channel. The dashed lines represent the statistical variation in the linear fit and are one standard deviation to either side. . . . . .

6.5 Efficiency of the $\chi_{x y}^{2}$ cut as measured for $D_{s} \rightarrow \phi \pi$ (above) and $B^{ \pm} \rightarrow$ $J / \psi K^{ \pm}$(below) compared to the same quantity in the corresponding Monte Carlo sample. . . . . . . . . . . . . . . . . . . 97

6.6 Plot of the $J / \psi$ mass in data and Monte Carlo for the electron channel. 98

7.1 Comparison of $B R\left(B^{ \pm} \rightarrow \phi K^{ \pm}\right)$calculated using the measured value of $B R\left(B^{ \pm} \rightarrow J / \psi K^{ \pm}\right) / B R\left(B^{ \pm} \rightarrow \phi K^{ \pm}\right)$with the values reported by BaBar[1], Belle[2], and CLEO[3]. . . . . . . . . . . . . . 102 


\section{List of Tables}

2.1 The fundamental fermions. Charges are in units of the absolute elec-

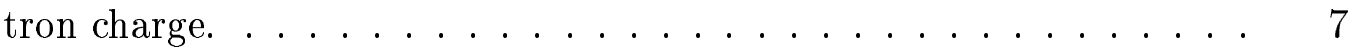

2.2 The quark content of various hadrons. . . . . . . . . . . 8

2.3 The forces and their corresponding bosons. The strength roughly gives the relative magnitudes in the case where two protons are just in

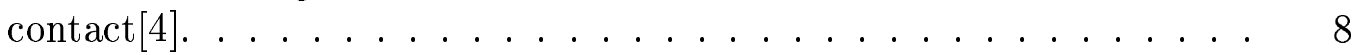

2.4 The Standard Model primitive vertices which involve fermions. Primitive vertices that involve only bosons also exist, but are not shown. . 13

5.1 Efficiencies for the eight representative runs in Monte Carlo simulation. 70

5.2 Efficiencies for the eight representative runs in Monte Carlo simulation. 71

5.3 Efficiencies for the eight representative runs in Monte Carlo simulation. 72

5.4 Parameters from the fit of the $d E / d x$ pull distributions using the lognormal function written above (second and third column). The fourth and fifth column list the mean and variance of the pulls. . . . . . .

5.5 Cuts used in the $\mathrm{dE} / \mathrm{dx}$ analysis. $\Delta \cot (\vartheta)$ is the difference between the polar angle cotangent of the two tracks and $\Delta x y$ is the separation between the two track helices in the transverse plane. . . . . . . . .

5.6 Results of fit to determine $\mu \mu$ fraction in $B \rightarrow J / \psi K$ signal using the 4-dimensional method. . . . . . . . . . . . . 86

5.7 Comparison of the estimates for the muon fraction in the $B^{ \pm} \rightarrow$ $J / \psi K^{ \pm}$signal region. Only statistical errors are quoted. $\ldots \ldots .88$

6.1 Contributions to the relative systematic uncertainty. . . . . . . . . 91

6.2 Parameters of the linear fit to the isolation efficiency as a function of $B^{ \pm}$meson $p_{T} \ldots \ldots \ldots \ldots \ldots \ldots \ldots \ldots \ldots$ 
I keep six honest serving-men

(They taught me all I knew);

Their names are What and Why and When

And How and Where and Who.

- Rudyard Kipling

xi 


\section{Chapter 1}

\section{Introduction}

\subsection{Historical Background}

The first mention of an atomic theory is by Leucippus and Democritus in the 5th century B.C. They philosophized that nature consisted of an infinite number of small, indivisible particles. They called these particles "atoms" which means "unable to be divided".

A more scientific approach to atomic theory was taken up in the 19th Century, when J.Dalton developed his atomic hypothesis to explain Chemical phenomenon. Shortly afterward, D.Mendeleev's periodic table of the elements strikingly demonstrate the success of the atomic theory. For a time, atoms were thought to be the fundamental building blocks of nature. Then, in 1897, J.J.Thomson discovered negatively charged particles that were ejected from different atoms, but which always had the same charge-to-mass ratio. He postulated that these particles were of a single type-a building block common to all atoms. Later, this sub-atomic particle would come to be known as the electron. Since atoms are electrically neutral the existence of this negatively charged ingredient implied a positively charged counterpart. Early in the 20th century E.Rutherford used scattering experiments to demonstrated the existence of a dense, positively charged central region inside the atom, which he called the nucleus. He would go on to experimentally verify the existence of the positively charged proton contained therein. In 1932 J.Chadwick identified an additional, neu- 
tral component inside the nucleus. He called this particle the neutron.

Exciting theoretical breakthroughs were also made during the beginning of the 20th Century. Quantum Theory emerged in 1900 when M.Planck used the idea of quantized energy to explain the radiation spectrum emitted by a black body. In 1905 A.Einstein would take the next great deductive leap by postulating the existence of the photon-a particle of light-to explain the photoelectric effect. In answer to Einstein's assignment of a particle nature to light, which is a wave, L.deBroglie proposed a theory whereby particles would have wave-like properties. N.Bohr would use the quantum behavior of electrons in explaining the phenomenon of discrete atomic spectra in 1913. Many other great scientists, such as Schrodinger, Heisenberg, and Born, would also contribute to theory of the subatomic world. One of the most astonishing predictions during this time was made by P.A.M.Dirac, who predicted the existence of antimatter in 1930. This prediction was verified two years later by C.Anderson when he discovered the positron (the anti-matter version of the electron) during his study of cosmic rays.

In the mid 1930's matter was thought to be composed entirely of protons, neutrons, and electrons. This simple state of affairs was not to last, however. Cosmic ray studies led to the discovery of the muon in 1937 and the discovery of pions in 1947. The advent of nuclear reactors and man-made particle accelerators led to the discovery of many more new particles. By the 1960's more than a hundred different particles had been discovered, which led to the question: "Could all these particles really be fundamental?" In 1964 Gell-Mann and Zweig came up with a theory ordering the particles in a manner akin to Mendeleev's ordering of the elements, albeit decidedly more complicated. Many of the particles could be described as being made of a more fundamental building block: the quarks. Particles containing quarks are called hadrons. Of the hadrons there are two types: mesons which are made of a quark and an anti-quark, and baryons which are made of a set of three quarks (or three anti-quarks). Initially, there were only three known quarks: the "up", "down", and "strange" quarks. A fourth quark was predicted to explain some experimental observations. This fourth "charm" quark was found in 1974, with the discovery of the $J / \psi$ particle, a meson made from a charm quark/anti-quark pair. This discovery 
was made independently by both B.Richter and S.Ting-hence the two names: $J$ and $\psi$. Since that time, "bottom" and "top" quarks have also been discovered. There are also particles not made up of quarks, that are called leptons.

Today, the world is thought to be made of six quarks and six leptons plus their anti-particles, along with the particles that mediate the four known forces: gravity, electromagnetism, the weak force, and the strong force. These particles and their interactions are described by the Standard Model of Particle Physics.

\section{$1.2 \quad$ High Energy Physics}

Particle physicists study the fundamental constituents of matter. These particles do not exist on the same energy and time scales as human life. They are too small to be "seen", even by the strongest of microscopes. To probe shorter distance scales, higher energies are needed. Also, some of the particles of interest are extremely heavy by subatomic standards. From Einstein's famous relation $E=m c^{2}$, we can see that greater energies are needed to make these more massive particles. This is why the study of fundamental particles is referred to as High Energy Physics.

Particle accelerators are one of the tools that high energy physicists use in their studies. The Tevatron at Fermilab is one such accelerator, and is currently the highest energy accelerator in the world. In the Tevatron, protons and anti-protons are accelerated toward each other at nearly the speed of light. The energy released in these collisions creates showers of particles. Many of these particles have extremely short lifetimes, from anywhere around $10^{-12} \mathrm{~s}$ to $10^{-25} \mathrm{~s}$, before decaying into other particles (which may also decay in turn). A detector is used to measure and store information on the decay products. This data is then used by particle physicists in an attempt to reconstruct information about the original particle. The Collider Detector at Fermilab is one such detector.

While the Standard Model of Particle Physics has proven wildly successful, it does have some limitations. For example, it cannot explain why there are six quarks and six leptons, why the fundamental particles have the masses that they do, or why there is more matter observed in the Universe than antimatter. There must be new physics 
beyond the Standard Model, and it is up to high energy physicists to find it.

\subsection{Outline of this Thesis}

This thesis describes a measurement of the ratio of branching ratios for the decay modes $B^{ \pm} \rightarrow \phi K^{ \pm}$and $B^{ \pm} \rightarrow J / \psi K^{ \pm}$. In Chapter 2 the theoretical background and motivation for this measurement will be introduced. A description of the experimental apparatus used for this measurement will be given in Chapter 3, where both the Tevatron and the Collider Detector at Fermilab will be discussed. In Chapter 4 the data and Monte Carlo samples used in this analysis will be described. The methodology used in the analysis will be described in detail in Chapter 5. In Chapter 6 the systematic uncertainties associated with the measurement will be discussed. The final results and conclusions will be presented in Chapter 7, and compared to similar measurements reported by the $e^{+} e^{-}$collider experiments BaBar[1], Belle[2], and CLEO[3]. 


\section{Chapter 2}

\section{Theoretical Motivation}

\subsection{The Standard Model of Particle Physics}

The Standard Model of Particle Physics is the most successful theory to date for describing particles and their interactions. According to the Standard Model all matter is built from a small number of fundamental, spin $\frac{1}{2}$ particles called fermions interacting via the exchange of gauge bosons. These fundamental fermions consist of six quarks and six leptons (and their anti-particles) organized into three families as shown in Table 2.1.

\subsubsection{The Fundamental Fermions}

The leptons all have an integral value for their electric charge. The muons $(\mu)$ and taus $(\tau)$ are heavier versions of the familiar electron $(e)$, having the same spin and electric charge. Being heavier than electrons, they are unstable and can decay into electrons and neutrinos (or other particles). The neutral leptons are called neutrinos. They were first postulated by Pauli in 1930 to account for the missing energy and momentum, being carried away by the undetected neutrinos, in nuclear $\beta$-decay[4]. The first direct detection of neutrinos was by Cowan and Reines in 1956, when they used the high flux of neutrinos produced at the Savannah River nuclear reactor in South Carolina to see "inverse" beta-decay reactions[5]. There is one 'flavor' of neu- 
trino associated with each 'flavor' of lepton. The lepton flavor is conserved in the Standard $\operatorname{Model}^{1}$, i.e. while the number of, say, electrons may not be conserved, the number of electrons plus electron neutrinos is. Similarly for the number of muons plus muon neutrinos, and the number of taus plus tau neutrinos.

The quarks all have fractional charges of either $+\frac{2}{3}$ or $-\frac{1}{3}$. While bare leptons can exist freely, quarks apparently can not. Quarks contain an extra degree of freedom in addition to their spin and electric charge called the color charge. They can come in one of three colors: red, blue, and green. This is not meant as the literal color, but rather as a method of labeling the three charges, just as 'plus' and 'minus' are used to label the two values of the electric charge. The parallel with color comes from the fact that red, blue, and green light combines to make white light, which is exactly the requirement for a stable combination of quarks-the combination must be colorless. Stable quark combinations consist of either three quarks of each of the three colors, or a quark and an anti-quark of matching color and anti-color ${ }^{2}$. The former are called baryons, and the latter are called mesons. All particles made up of quarks are collectively referred to as hadrons. Table 2.2 gives the quark content for several different hadrons.

\subsubsection{Interactions}

In the Standard Model interactions between the fundamental fermions take place via the exchange of bosons. There are bosons corresponding to each of the four forces in nature: electromagnetism, the strong nuclear force, the weak nuclear force, and gravity. Some properties of the different force carriers are summarized in Table 2.3.

The electromagnetic force is responsible for most extra-nuclear physics. It is responsible for the binding of electrons to nuclei, and therefore all known chemistry.

\footnotetext{
${ }^{1}$ Recent evidence suggests that neutrino oscillations, oscillations of one flavor of neutrino into another, do occur. While rarely observed, this would allow interactions that do not conserve the lepton number. A possible solution would be to insert another CKM-like matrix into the Standard Model, but for the lepton sector. The CKM matrix will be discussed in Section 2.3

${ }^{2}$ Other, more complicated, colorless combinations of quarks are postulated. Several experiments have recently reported signals consistent with a particle having a pentaquark structure of four quarks and one anti-quark[6].
} 


\begin{tabular}{|c|c|c|c|c|}
\hline Quarks & Symbol & Charge & \multicolumn{2}{|c|}{ Mass $(\mathrm{MeV})$} \\
\hline up, & $u$ & $+\frac{2}{3}$ & $1.5-5$ & \\
\hline down, & $d$ & $-\frac{1}{3}$ & $3-9$ & \\
\hline charm, & $c$ & $+\frac{2}{3}$ & $(1.1-1.4)$ & $\times 10^{3}$ \\
\hline strange, & $s$ & $-\frac{1}{3}$ & $75-170$ & \\
\hline top, & $t$ & $+\frac{2}{3}$ & $(173.8 \pm 5.2)$ & $\times 10^{3}$ \\
\hline bottom, & $b$ & $-\frac{1}{3}$ & $(4.1-4.4)$ & $\times 10^{3}$ \\
\hline Leptons & Symbol & Charge & Mass (Me & \\
\hline electron, & $e$ & -1 & 0.511 & \\
\hline electron neutrino, & $\nu_{e}$ & 0 & $\approx 0$ & \\
\hline muon, & $\mu$ & -1 & 105.7 & \\
\hline muon neutrino, & $\nu_{\mu}$ & 0 & $<0.17$ & \\
\hline tau, & $\tau$ & -1 & 1777 & \\
\hline tau neutrino, & $\nu_{\tau}$ & 0 & $<18.2$ & \\
\hline
\end{tabular}

Table 2.1: The fundamental fermions. Charges are in units of the absolute electron charge. 


\begin{tabular}{|cc||cc|}
\hline Hadron & Quark Content & Hadron & Quark Content \\
\hline$B^{+}$ & $\bar{b} u$ & $K^{+}$ & $\bar{s} u$ \\
$B^{-}$ & $b \bar{u}$ & $K^{-}$ & $s \bar{u}$ \\
$\phi$ & $\bar{s} s$ & $K^{0}$ & $\bar{s} d$ \\
$J / \psi$ & $\bar{c} c$ & $\bar{K}^{0}$ & $s \bar{d}$ \\
$D_{S}^{+}$ & $c \bar{s}$ & $\pi^{+}$ & $u \bar{d}$ \\
$D_{S}^{-}$ & $\bar{c} s$ & $\pi^{-}$ & $\bar{u} d$ \\
$D^{+}$ & $c \bar{d}$ & $\pi^{0}$ & $(\bar{u} u-\bar{d} d) / \sqrt{2}$ \\
$D^{-}$ & $\bar{c} d$ & $p$ & $u u d$ \\
$D^{0}$ & $c \bar{u}$ & $n$ & $u d d$ \\
$\bar{D}^{0}$ & $\bar{c} u$ & $\Lambda^{0}$ & $u d s$ \\
\hline
\end{tabular}

Table 2.2: The quark content of various hadrons.

\begin{tabular}{|lccc|}
\hline Force & Mediator & Spin/Parity & Strength \\
\hline Strong Force & Gluon, $g$ & $1^{-}$ & 1 \\
Electromagnetism & Photon, $\gamma$ & $1^{-}$ & $10^{-2}$ \\
Weak Force & $W^{ \pm}, Z^{0}$ & $1^{-}, 1^{+}$ & $10^{-7}$ \\
Gravity & Graviton & $2^{+}$ & $10^{-39}$ \\
\hline
\end{tabular}

Table 2.3: The forces and their corresponding bosons. The strength roughly gives the relative magnitudes in the case where two protons are just in contact[4]. 
It is mediated by a massless, spin-1 boson called the photon which couples to all particles possessing an electric charge.

The strong nuclear force is responsible for the binding of quarks into hadrons and the binding of protons and neutrons inside of a nucleus. It is mediated by massless, spin-1 bosons called gluons. In strong interactions the color charge has a role analogous to that of the electric charge in electromagnetic interactions. Unlike the photons in electromagnetism, gluons themselves carry (color) charge and can couple directly to other gluons. Each gluon carries both a color and an anti-color. There are eight types of gluon corresponding to each of the states in a color octet.

The weak nuclear force is responsible for nuclear $\beta$-decay, and is responsible for all flavor changing interactions. It is mediated by three massive bosons called the $W^{+}, W^{-}$, and $Z^{0}$. The superscript denotes each boson's electric charge. Since the force carriers are so massive $\left(M_{W}=80.2 \mathrm{GeV}, M_{Z}=91.2 \mathrm{GeV}\right)$ the weak force has an extremely short range.

Gravity is by far the weakest force on a particle scale, and it's effects are negligible at particle accelerator energies. The reason gravity is so prevalent on a cosmic scale is that it's effects are cumulative-there is no negative gravitational charge (mass). Gravity is not incorporated into the Standard Model and is only included here for completeness. The existence of a massless, spin-2 boson called the graviton has been postulated as the gravitational force mediator. Developing an effective quantum theory for gravity is still one of the primary goals in modern Physics.

The existence of four distinct, unrelated interaction fields seems mathematically unrefined. Since Einstein's time, Physicists have been trying to develop a theory that treats the four forces as different aspects of one, unified field-much in the same way that electricity and magnetism are joined in electromagnetic theory. A first step along this path was taken by Glashow, Weinberg, and Salam in the late 1960's when they developed a highly successful theory unifying the electromagnetic and weak forces into one electroweak force, wherein they predicted both the existence and masses of the $W^{ \pm}$and $Z^{0}$ bosons[4]. Their theory will be more fully explored in later sections. Numerous attempts have also been made to include the strong force and gravity in a unification scheme, but so far no satisfactory theory has been developed. 


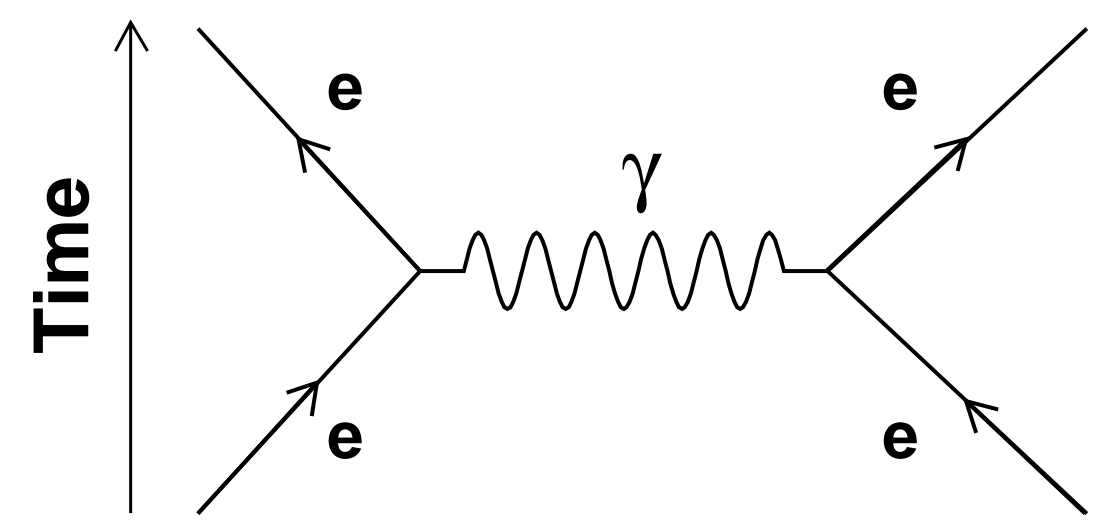

Figure 2.1: Feynman diagram representing the scattering interaction of two electrons via photon exchange.

\subsection{Feynman Diagrams}

A brief discussion of Feynman diagrams is now in order. The goal of this section is not to give the reader a detailed understanding of Feynman diagram calculations, but rather a basic working knowledge of what the diagrams represent. For a complete description of Feynman diagrams see any Quantum Field Theory text, e.g. [7].

Fig. 2.1 shows a typical Feynman diagram. The solid lines represent fermionselectrons in this case-and the wavy line represents a photon. The arrows on the fermion lines show the direction of matter flow. A particle line running "backward in time" would represent the corresponding anti-particle moving forward in time. Fig. 2.1 then represents two electrons scattering off of each other by exchanging a photon. Which electron emits and which absorbs the photon is not shown, the diagram represents both processes.

In such diagrams external lines represent real, observable particles. Internal lines, however, depict particles that cannot be observed without changing the process being represented. They are said to depict "virtual" particles. Energy and momentum is conserved at each vertex, but the mass of virtual particles is not required to be the same as it is in a free particle state, i.e. it can assume whatever value is needed to satisfy energy and momentum conservation in the interaction. In essence, the external 


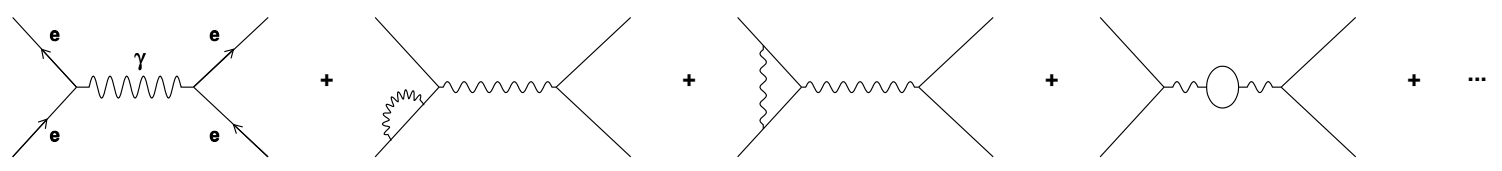

Figure 2.2: Sum of Feynman diagrams representing the electromagnetic scattering interaction between two electrons.

lines tell us what happened, and the internal lines give us a mechanism for how it happened.

Note that Feynman diagrams like those in Fig. 2.1 are purely symbolic, and do not represent actual particle trajectories. The vertical axis represents the flow of time, but the horizontal axis does not represent a spatial distribution. The diagram actually represents a number corresponding to the amplitude for this physical process to occur. For a specific particle interaction the sum of all possible Feynman diagrams must be added together as shown in Fig. 2.2. There are an infinite number of diagrams for any given process. They can be thought of as a series expansion around a ground state, where the higher order terms are those with more vertices. The Feynman rules for calculating the diagram amplitudes are derived from the Lagrangian describing the particle field interactions. A necessary requirement for any theory (Lagrangian) to be useful is that it's Feynman series must converge, i.e. it must be renormalizable ${ }^{3}$

While there are an infinite number of different possible Feynman diagrams they can all be constructed from a relatively small number of fundamental, or primitive, vertices determined by the theory. The diagrams in Table 2.4 (along with their topological equivalents) represent all of the Standard Model primitive vertices that involve fermions. Fig.(A) shows an electrically charged fermion emitting or absorbing a photon. Fig.(B) shows a color charged fermion (quark) emitting or absorbing a gluon. The gluons in Feynman diagrams are represented by curly lines. Fig.(C) shows a charged lepton converting into it's corresponding neutrino by emission of a $W^{-}$. The weak bosons $\left(W^{ \pm}\right.$and $\left.Z^{0}\right)$ in Feynman diagrams are represented by dashed

\footnotetext{
${ }^{3}$ Many theories suffer from divergences, but the resultant infinities can be successfully "defined away", thus salvaging their usefulness. This process is called renormalization. A non-renormalizable theory is one in which no mathematical mechanism exists to remove it's divergences.
} 
lines. In the case of Fig.(D) a down-type quark (a quark of type $d, s$ or $b$ ) is converted into an up-type quark (a quark of type $u, c$, or $t$ ), by emitting a $W^{-}$. Since the $W^{+}$ is the anti-particle of the $W^{-}$, the processes in (C) and (D) can also be interpreted as taking place through the absorption of a $W^{+}$. Notice that in all the cases involving a $W^{ \pm}$the electric charge is still conserved at the vertex, even though the incoming and outgoing fermions have different charges. Fig.(E) shows a fermion emitting or absorbing a $Z^{0}$.

\subsection{Weak Interactions and the CKM Matrix}

As has already been stated, weak vertices involving a $W^{ \pm}$change the flavor of the fermion involved. For leptons the mixing takes place strictly within the generations (in the Standard Model, see the footnote on page 6).

$$
\left(\begin{array}{c}
\nu_{e} \\
e
\end{array}\right) \quad\left(\begin{array}{c}
\nu_{\mu} \\
\mu
\end{array}\right) \quad\left(\begin{array}{c}
\nu_{\tau} \\
\tau
\end{array}\right)
$$

i.e. while an electron can convert into an electron neutrino, it cannot convert into a muon neutrino.

For quarks some inter-generation mixing does occur. It is described by rotating the down-type quark mass states into weak states,

$$
\left(\begin{array}{c}
d^{\prime} \\
s^{\prime} \\
b^{\prime}
\end{array}\right)=\left(\begin{array}{ccc}
V_{u d} & V_{u s} & V_{u b} \\
V_{c d} & V_{c s} & V_{c b} \\
V_{t d} & V_{t s} & V_{t b}
\end{array}\right)\left(\begin{array}{l}
d \\
s \\
b
\end{array}\right)
$$

such that the weak mixing takes place between the states:

$$
\left(\begin{array}{l}
u \\
d^{\prime}
\end{array}\right) \quad\left(\begin{array}{l}
c \\
s^{\prime}
\end{array}\right) \quad\left(\begin{array}{c}
t \\
b^{\prime}
\end{array}\right)
$$


(A) An electrically charged fermion emitting or absorbing a photon.

(B) A color charged fermion (quark) emitting or absorbing a gluon.

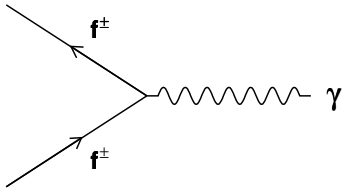

(C) An electrically charged lepton converts into it's corresponding neutrino, with emission of a $W^{-}$.

(D) A down-type quark converts into an up-type quark, with emission of a $W^{-}$.

(E) A fermion emitting or absorbing a $\mathrm{Z}$ boson.
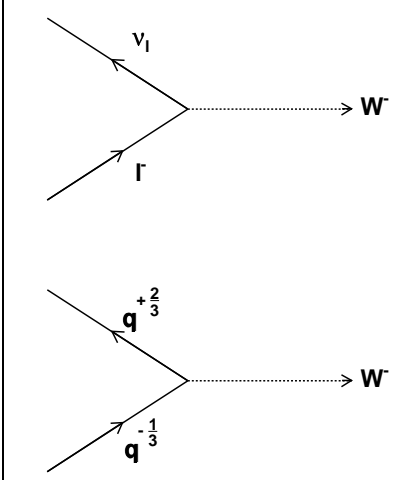

(E) A fermion emitting or absorb-
ing a $\mathrm{Z}$ boson.

Table 2.4: The Standard Model primitive vertices which involve fermions. Primitive vertices that involve only bosons also exist, but are not shown. 


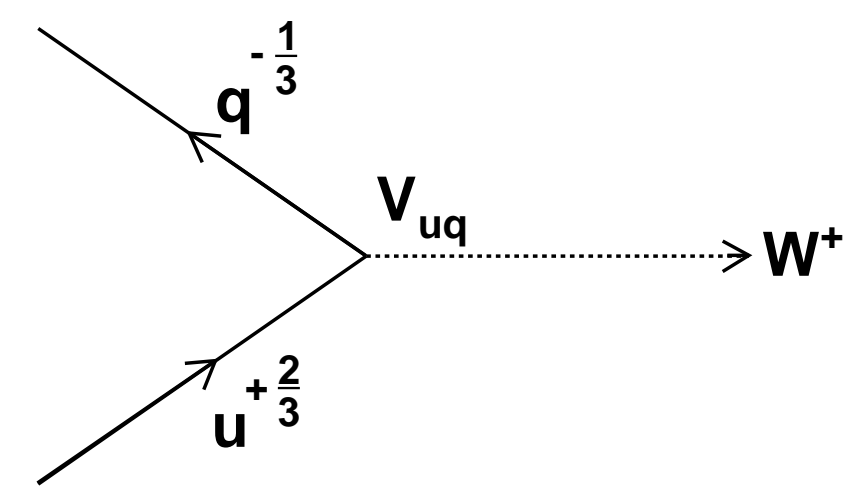

Figure 2.3: Vertex showing an ingoing up quark with an outgoing $W^{+}$and outgoing quark $q$, where $q=d, s$, or $b$. This vertex must be multiplied by the element $V_{u q}$ of the CKM matrix describing the overlap of the emitted quark with the weak state partnered with the $u$.

The matrix relating the quark mass states to their weak states is called the CabbiboKobayashi-Maskawa (CKM) matrix.

For example, the transition of an up quark into a $W^{+}$and another quark can occur through one of three vertices as shown in Fig. 2.3, where $q$ can be either $\mathrm{d}$, $\mathrm{s}$, or $\mathrm{b}$. These vertices must be multiplied by an additional term describing the overlap of the emitted quark with the weak state partnered with the $\mathrm{u}$, namely $V_{u q}$ from the CKM matrix. The nine complex entries in the CKM matrix are not all independent. To ensure proper normalization and the conservation of quark number the CKM matrix must be unitary.

The need for unitarity imposes three real and three complex equations of constraint on the nine complex terms of the CKM matrix. This leaves three real parameters and six complex phases undetermined. By judicious redefinition of the quark phases, five of the complex phases in the CKM matrix can be effectively removed. This leaves three real parameters and one complex phase that must be determined experimentally. The existence of this irremovable complex phase is important as it provides a theoretical mechanism for $\mathrm{CP}$ violation, which will be further explored in the next section. 
Various parameterizations of the CKM matrix are possible. One such parametrization is

$$
\left(\begin{array}{ccc}
c_{1} & s_{1} c_{3} & s_{1} s_{3} \\
-s_{1} c_{2} & c_{1} c_{2} c_{3}-s_{2} s_{3} e^{i \delta} & c_{1} c_{2} s_{3}+s_{2} c_{3} e^{i \delta} \\
& & \\
-s_{1} s_{2} & c_{1} s_{2} c_{3}+c_{2} s_{3} e^{i \delta} & c_{1} s_{2} s_{3}-c_{2} c_{3} e^{i \delta}
\end{array}\right)
$$

where $s_{i}$ stands for $\sin \left(\theta_{i}\right)$ and $c_{i}$ stands for $\cos \left(\theta_{i}\right)$. A popular, alternative parametrization first proposed by Wolfenstein is

$$
\left(\begin{array}{ccc}
1-\lambda^{2} / 2 & \lambda & A \lambda^{3}(\rho-i \eta) \\
-\lambda & 1-\lambda^{2} / 2 & A \lambda^{2} \\
A \lambda^{3}(1-\rho-i \eta) & -A \lambda^{2} & 1
\end{array}\right)
$$

\subsection{CP Violation}

Until the 1950's it was believed that physical processes were invariant under certain discrete symmetry transformations. Specifically, they were believed to be invariant under charge conjugation where all charges are reversed, effectively replacing every particle with its corresponding antiparticle; parity where all spatial coordinates are inverted through the origin (mirror symmetry); and time reversal. These symmetry operations are denoted as $\mathrm{C}, \mathrm{P}$, and $\mathrm{T}$, respectively.

In 1956 experiments by C.S. Wu demonstrated that $\mathrm{P}$ was not conserved in beta decays within cobalt 60 (in fact it was maximally violated). Further investigation showed that, while parity was conserved in reactions involving the strong and electromagnetic forces, parity violation was quite common in reactions involving the weak 
force [5]. The invariance of reactions under $\mathrm{C}$ would come into question shortly afterward when investigation of the decays

$$
\pi^{+} \rightarrow \mu^{+}+\nu_{\mu}
$$

and

$$
\pi^{-} \rightarrow \mu^{-}+\bar{\nu}_{\mu}
$$

revealed that all neutrinos (anti-neutrinos) are created with left (right) handed helicities in the center of mass frame, where helicity is defined as the spin orientation of the particle around the axis in the direction of its motion. While these reactions clearly violate parity, they also violate charge conjugation as well. The action of $\mathrm{C}$ on equation (2.6) transforms it into equation (2.7), but with a left handed $\mu$ and $\bar{\nu}_{\mu}$, a decay which does not seem to appear in nature. Notice, however, that under the combined operation of CP equation (2.6) is transformed into equation (2.7) with the same helicities as those observed experimentally[8].

While neither $\mathrm{C}$ nor $\mathrm{P}$ is conserved in weak interactions it was thought for a time that the combined operation, CP, was. This lead to some rather interesting predictions by Gell-Mann and Pais concerning the neutral kaon system[4]. First, they showed that oscillations of $K^{0} \leftrightarrow \bar{K}^{0}$ were theoretically possible. Further, due to the strange quark content of the $\mathrm{K}$ mesons and kinematic restrictions, these particles could only decay weakly. This lead them to predict that the neutral kaons, produced by the strong force in mass eigenstates, would decay weakly as two different particles

$$
K_{1}=\frac{1}{\sqrt{2}}\left(\left|K^{0}>-\right| \bar{K}^{0}>\right)
$$

and

$$
K_{2}=\frac{1}{\sqrt{2}}\left(\left|K^{0}>+\right| \bar{K}^{0}>\right)
$$

which were eigenstates of CP. The difference would be most noticeable in the different mean decay rates of these two particles. As incredible as this prediction sounds, evidence for the $K_{2}$ was discovered by Lederman in 1956[5]. 
While this seemed like striking confirmation of the idea of combined CP invariance, the euphoria was not long lived. In 1964 Cronin and Fitch observed CP violating decays of the longer lived $\mathrm{K}$ mesons. Apparently, the long-lived kaons were not the pure CP eigenstate given as $\mid K_{2}>$, but rather also contained a small amount of $\mid K_{1}>$.

$$
\mid K_{L}>=\frac{1}{\sqrt{1+|\epsilon|^{2}}}\left(\left|K_{2}>+\epsilon\right| K_{1}>\right)
$$

This CP violation, however, was only a very small effect, $\epsilon \approx 2.3 \times 10^{-3}$, as opposed to the maximal violation seen for $\mathrm{C}$ and $\mathrm{P}$ invariance separately[5]. Like the kaon system, the B sector also has decays that are limited, by kinematics and the fact that quark content is conserved in strong and electromagnetic interactions, to decay only weakly. It is hoped that study of the B meson system will lead to more accurate measurements of the CKM matrix elements and shed new light on the origins of CP violation.

This thesis presents evidence for the decay modes

$$
B^{ \pm} \rightarrow \phi K^{ \pm}
$$

and finds the relative branching ratio with regards to the decay $B^{ \pm} \rightarrow J / \psi K^{ \pm}$. It is intended that, once more data become available, the techniques used in this measurement will be used to measure the asymmetry between the two CP conjugate decays,

$$
\mathcal{A}_{\mathcal{C P}}=\frac{\operatorname{rate}\left(B^{+} \rightarrow \phi K^{+}\right)-\operatorname{rate}\left(B^{-} \rightarrow \phi K^{-}\right)}{\operatorname{rate}\left(B^{+} \rightarrow \phi K^{+}\right)+\operatorname{rate}\left(B^{-} \rightarrow \phi K^{-}\right)}
$$

A more detailed discussion of the origin of $\mathrm{CP}$ violation in this channel is therefore appropriate. The origin of the $\mathrm{CP}$ violation just discussed in the kaon system stems from the mixing of the two neutral particles, $K^{0}$ and $\bar{K}^{0}$, and is a form of 'indirect' $\mathrm{CP}$ violation. For charged decays such as those in (2.11) no such mixing can occur between the initial states, but 'direct' $\mathrm{CP}$ violation is possible. 
In charged decays, $\mathrm{CP}$ violation takes place through the interference of two (or more) amplitudes, i.e. an interference between the different Feynman Diagrams for the same decay process. The amplitude for a particular Feynman diagram can be written as

$$
A \mathrm{e}^{i \phi} \mathrm{e}^{i \delta}
$$

where $A$ is the magnitude of the diagram's amplitude, $\phi$ is the weak interaction phase shift originating from the irremovable complex phase in the CKM matrix, and $\delta$ is the strong interaction phase shift which occurs when the diagram contains an absorptive part $^{4}[9]$. A diagram has an absorptive part whenever two propagators (internal lines) can simultaneously go on mass-shell within the momentum region being considered $[7]$.

If we were to look at the diagram for the CP conjugate process, we would see that the values for $A$ and $\delta$ would remain the same, while $\phi$ would undergo a change of sign due to the complex conjugation of the CKM matrix (due to C). In other words:

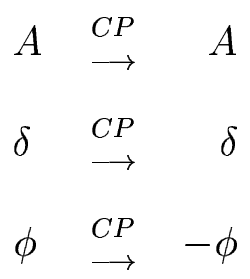

If we were to now consider a process that had contributions from multiple amplitudes, the total amplitude would be the sum

$$
A_{t o t}=\sum_{i} A_{i} \mathrm{e}^{i \phi_{i}} \mathrm{e}^{i \delta_{i}}
$$

Notice that under CP the magnitude of each contributing process would remain the same, but the relative phases between them would, in general, change (provided that $\phi_{i} \neq \phi_{k}$ and $\delta_{i} \neq \delta_{k}$ for some $i$ and $k$ ). This change in the relative phases of the contributing amplitudes will result in different magnitudes of $A_{t o t}$ for the CP conjugate decays. This is shown schematically in Fig. (2.4) for the case of only

\footnotetext{
${ }^{4}$ This is often referred to as final state rescattering in the literature.
} 

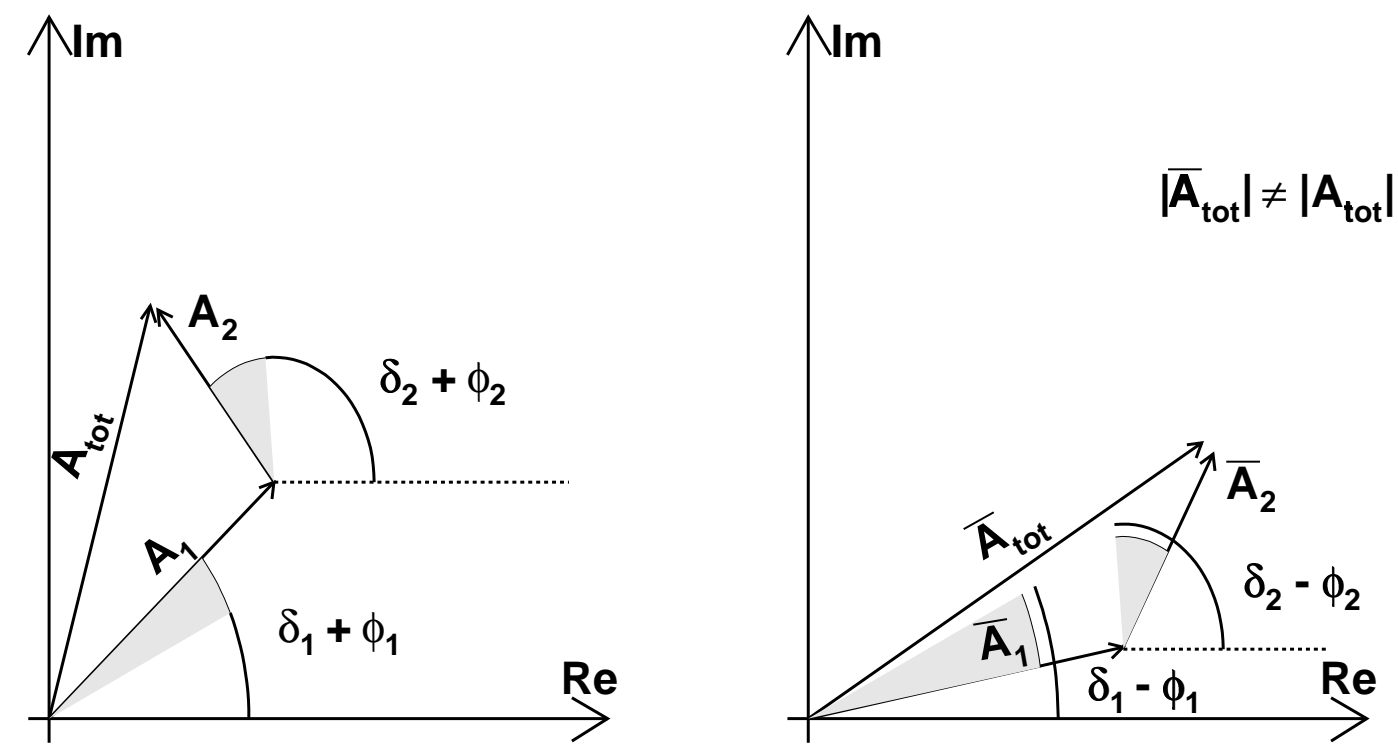

Figure 2.4: A schematic representation of the interference responsible for CP violation. The strong phase angles are represented by $\delta_{i}$, and the weak phase angles are represented by $\phi_{i}$, for each amplitude, $A_{i}$. The left diagram shows the complex sum for the two amplitudes, $A_{1}+A_{2}$. The right diagram shows the complex sum of the $\mathrm{CP}$ conjugate amplitudes, $\bar{A}_{1}+\bar{A}_{2}$. Notice that while $\left|A_{1}\right|=\left|\bar{A}_{1}\right|$ and $\left|A_{2}\right|=\left|\bar{A}_{2}\right|$, the total amplitudes are different for the two cases, i.e. $\left|A_{1}+A_{2}\right| \neq\left|\bar{A}_{1}+\bar{A}_{2}\right|$.

two contributing amplitudes. Also note that, if $A_{t o t}$ were dominated by one of its component amplitudes, i.e. there existed an $A_{k}$ such that $A_{k} \gg A_{i}$ for all $i \neq k$, then $\mathrm{CP}$ violation would still occur for the decay in question but at such a small rate as to be difficult-if not impossible-to measure.

To recapitulate, for $\mathrm{CP}$ violation to occur:

1. The CKM matrix must contain an irremovable complex phase.

2. The decay in question must have contributions from two (or more) diagrams with different CKM phases.

3. One of the interfering amplitudes must have a strong phase shift.

Requirements (1) and (2) are needed to get the different weak phase angles[10].

The decay (2.11) is dominated by the penguin diagram show in Fig. (2.5). This diagram actually represents three different amplitudes since the internal quark lines 


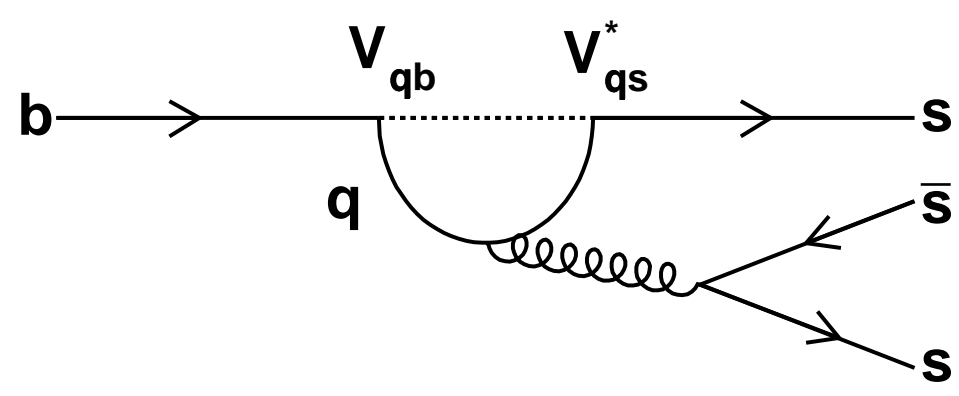

Figure 2.5: The Feynman Diagram for $b \rightarrow s \bar{s} s$. This actually represents three different amplitudes, since different quarks may participate in the internal lines of the loop; $q=u, c$, or $t$.

can be any of the up-type quarks, $q=(u, c$, or $t)$. The weak phase angle comes from the CKM terms applied at each of the two weak vertices, and the strong phase angle becomes non-zero when the center of mass energy of the process is great enough for the internal quark lines to go above mass shell[9].

Both indirect and direct $\mathrm{CP}$ violation have been observed in B meson decay channels. Indirect $\mathrm{CP}$ violation for a $\mathrm{B}$ meson decay was first reported in the channel $B^{0} \rightarrow J / \psi K^{0}[11,12]$. Direct CP violation in a B meson decay was first reported for the channel $B^{0} \rightarrow K^{ \pm} \pi^{\mp}[13,14]$. CP violation measurements have since been made in several other channels, the goal being to over-constrain the parameters of the CKM matrix and test the validity of the CKM theory.

In the Standard Model $\mathcal{A}_{\mathcal{C P}}\left(B^{ \pm} \rightarrow \phi K^{ \pm}\right)$is expected to be zero. Since the decays $B^{ \pm} \rightarrow \phi K^{ \pm}$are almost pure penguin processes, new physics could be detected if exotic particles were to participate in the penguin loop. Some models speculate that new Physics contributions could lead to an asymmetry as high as order unity[15]. It would be interesting to measure this quantity and see how it compares with the Standard Model prediction and preliminary measurements recently reported by the BaBar Collaboration[16] (which are consistent with the Standard Model). 


\section{Chapter 3}

\section{The Experimental Setup}

\subsection{The Tevatron}

The Tevatron-originally known as the Energy Doubler/Saver-was built in the early 1980's at the Fermi National Accelerator Laboratory (Fermilab) in Batavia, Illinois. It has undergone several upgrades since then, increasing in both collision energy and luminosity. A major upgrade of the Tevatron took place between September 1997 and March 2001, with all subsequent operations being dubbed as 'Run II'. In Run II the Tevatron collides protons and antiprotons with a center of mass energy of $1.96 \mathrm{TeV}$. It is presently the highest energy particle collider in the world. The initial Run II integrated luminosity goal was $2 \mathrm{fb}^{-1}$ using instantaneous luminosities up to $2 \times 10^{32} \mathrm{~cm}^{-2} \mathrm{~s}^{-1}$, with a long-term (Run IIb) integrated luminosity goal of $8 \mathrm{fb}^{-1}[17]$. As of early 2004 the Tevatron has achieved a record luminosity of $5.9 \times 10^{31} \mathrm{~cm}^{-2} \mathrm{~s}^{-1}$. This analysis uses $200 \mathrm{pb}^{-1}$ of data collected as of Oct. 2003.

Fig. 3.1 shows a diagram of the Fermilab accelerator chain. Initially, electrical discharges in hydrogen gas are used to produce $\mathrm{H}^{-}$ions. A Cockroft-Walton tower is used to accelerate the $\mathrm{H}^{-}$ions up to $750 \mathrm{KeV}$ and send them into a 500 foot long linear accelerator called the Linac. The Linac uses cavities with time alternating electromagnetic fields to accelerate the ions up to $400 \mathrm{MeV}$. The $\mathrm{AC}$ nature of the Linac separates the continuous beam into bunches. The ions then enter the Booster ring, a synchrotron accelerator 475 meters in circumference. They are passed through 


\section{FERMILAB'S ACCELERATOR CHAIN}

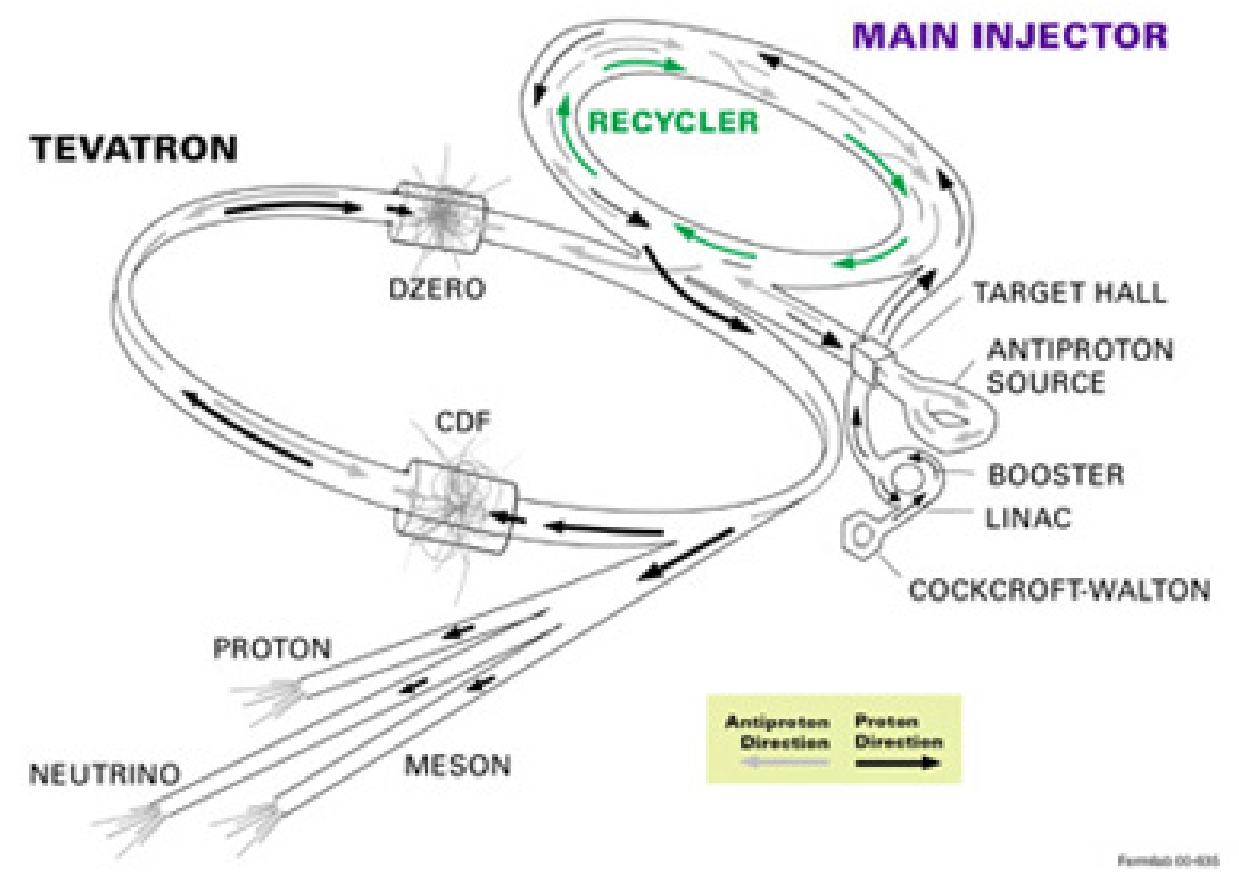

Figure 3.1: Diagram of the Fermilab accelerator chain. 
a thin carbon foil, stripping off the two electrons and leaving a bare proton. The protons are then accelerated in the Booster to $8 \mathrm{GeV}$. They then enter the Main Injector ring which ramps the protons up to $150 \mathrm{GeV}$ and prepares them to be injected into the Tevatron.

The Main Injector is also used to create antiprotons. It does so by directing protons onto a nickel target at $120 \mathrm{GeV}$. Many different types of particles are created by this process, so the antiprotons must be focused by a lithium lens and filtered out using a pulsed magnet. Roughly, one antiproton is created and successfully captured for every 50,000 protons incident on the target. After creation the antiprotons have a large energy spread, so they are first sent into the Debuncher where they are stochastically cooled before being sent to the Accumulator ring where they are stored and cooled further. After a sufficient number $\left[(80-200) \times 10^{10}\right]$ of antiprotons have been stored they are sent into the Main Injector where they are accelerated up to $150 \mathrm{GeV}$.

After the protons and antiprotons in the Main Injector reach $150 \mathrm{GeV}$ they are ready to be injected into the Tevatron. The Tevatron is the main accelerator ring and has a radius of one kilometer. It ramps the protons and antiprotons up to 980 $\mathrm{GeV}$. An advantage of using antiprotons is that they can be accelerated in the same ring as the protons (in the opposite direction), which results in a significant saving in construction costs. The drawback is that the luminosity is limited by the antiproton production rate. In the current operating mode the protons and antiprotons travel around the ring in $36 \times 36$ bunches each about 1 meter long and with a circular width of a few millimeters. At two sites along the Tevatron ring, CDF and D0, the two beams are focused by superconducting quadrapole magnets to a width of approximately $35 \mu \mathrm{m}$ and collided. This analysis was done using the CDF detector.

\subsection{Collider Detector at Fermilab (CDF)}

\subsubsection{Detector Overview}

The Collider Detector at Fermilab (CDF) is a general purpose, solenoidal detector capable of precision charged particle tracking, fast projective calorimetry, and fine 


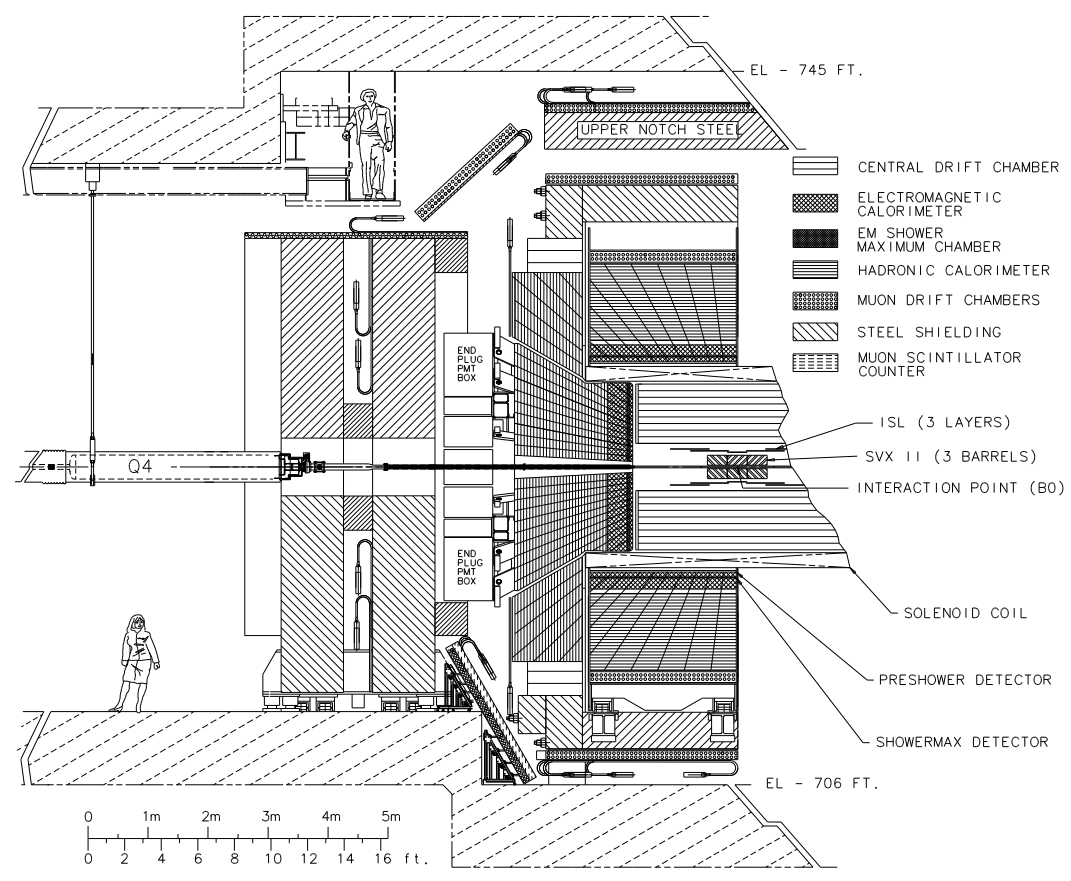

Figure 3.2: Cross-sectional view of the CDF detector.

grained muon detection. It is capable of making QCD, electroweak, and heavy flavor physics measurements, and can also be used to search for exotic, new particles. It is run by a multi-national collaboration of over 800 physicists from more than 50 different institutions. A schematic diagram of the CDF detector can be seen in Fig. 3.2.

The detector was designed to be nearly cylindrically symmetric around the beamline and forward-backward symmetric with respect to the nominal interaction point. The tracking systems are located within a superconducting solenoid that provides a 1.4 Tesla magnetic field in the direction of the beamline. The momentum and charge of tracked particles can then be determined by their deflection in the magnetic field. A Time-of-Flight (TOF) system is positioned directly outside (radially) of the tracking system. Time-of-flight information can be combined with momentum information from the tracking system to aid in particle identification. The calorimeter systems are located outside the TOF and are used to measure electron and photon energies, 
jet energies, and net transverse energy flow. The calorimeter systems are also used to identify electrons and photons. The muon detection systems are located furthest from the beamline and are used for triggering and to identify previously tracked particles as muons. Each of these systems will be described in further detail in the following sections.

\subsection{The Tracking System}

Efficient, precision charged particle tracking is critical for most CDF analysis. The tracking system is comprised of a drift chamber, called the Central Outer Tracker (COT), and a silicon based tracking system comprised of three parts: the Layer Zero Zero (L00), the Silicon Vertex Detector for Run II (SVX II), and the Intermediate Silicon Layer (ISL). The CDF tracking systems are located inside of a superconducting solenoid that produces a 1.4 Tesla magnetic field in the axial direction. The solenoid is cylindrical, and contains a region $2.8 \mathrm{~m}$ in diameter and $3.5 \mathrm{~m}$ long,

The trajectories of charged particles in a uniform magnetic field are bent to form a helix. The curvature of the helix can be used to determine the momentum of the particle and the sign of its electric charge. The CDF tracking systems are used to reconstruct the track trajectories of charged particles that pass through them. There are two coordinate systems commonly used at CDF: A cartesian coordinate system $(x, y, z)$ where the origin is located at the nominal particle interaction point, and the positive z-direction is along the beamline in the direction of proton motion with the x-direction pointing toward the outside of the Tevatron ring, and the ydirection pointing straight up perpendicular to the plane of the Tevatron ring; A polar coordinate system $(r, \theta, \phi)$ where $\mathrm{r}$ is the distance from the beamline, $\theta$ is the polar angle made with respect to the z-axis, and $\phi$ is the azimuthal angle a projection on the xy plane would make relative to the x-axis. Five track parameters are used at CDF to describe the trajectory of tracked particles:

- $z_{0}$ - The z-position of the track at its point of closest approach to the beamline.

- $d_{0}$ - The signed impact parameter of the track, the distance of closest approach 
to the beamline.

- $c$ - The half-curvature of the track, $c=\frac{1}{2 \rho}$ where $\rho$ is the radius of the circle made by a projection of the track onto the xy plane.

- $\phi_{0}$ - The azimuthal angle of the track at its point of closest approach to the beamline.

- $\cot \theta-$ The cotangent of the polar angle of the track at its point of closest approach to the beamline, also sometimes referred to as $\lambda$.

Some other common variables used at CDF (and other collider experiments) are the pseudorapidity defined as

$$
\text { pseudorapidity, } \eta=-\ln \left[\tan \left(\frac{\theta}{2}\right)\right]
$$

and the transverse energy and transverse momentum of a particle, $E_{T}=E \sin \theta$ and $p_{T}=p \cdot \sin \theta$, respectively[18].

A schematic diagram of the tracking system can be found in Fig. 3.3. COT tracks are combined with Silicon tracks and extrapolated back to the initial beam interaction point called the Primary Vertex (PV), allowing for a precise measurement of the momentum of charged particles. These reconstructed tracks can also be used to identify displaced (also called secondary) vertices created when a long-lived particle decays a discernible distance away from the PV where it was created. Along with being useful in identifying these long-lived particles, displaced vertices are also useful for triggering and suppressing the large inherent QCD background. Reconstructed tracks can also be combined with information from the TOF, calorimeter, and muon systems to aid in identifying the tracked particles.

\subsubsection{Silicon Tracking}

The innermost tracker, the L00, was added to the original CDF II design to increase the impact parameter resolution of the tracking system. It consists of 48 single-sided silicon wafers mounted directly on the beam pipe. The wafers come in 


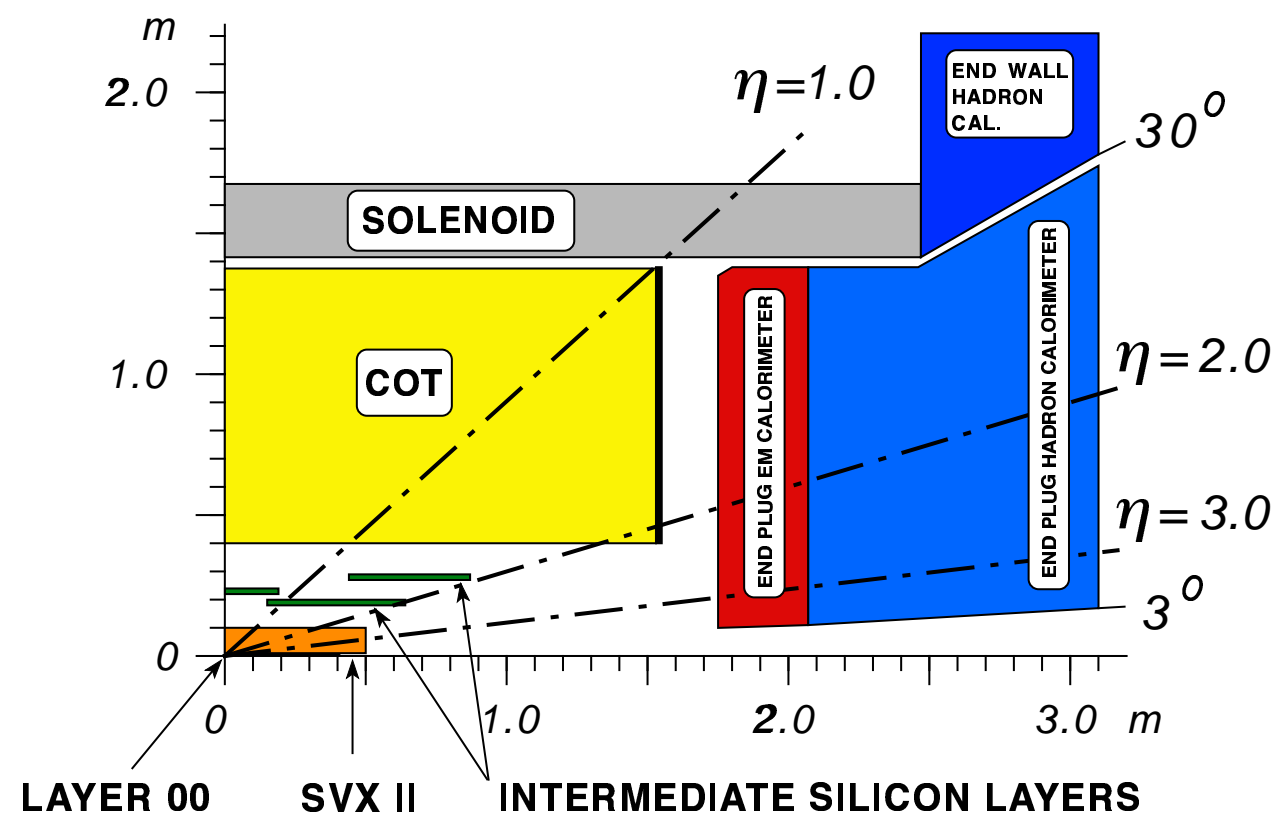

Figure 3.3: Cross-sectional view of the CDF tracking system for Run II, along with the plug calorimeter systems.

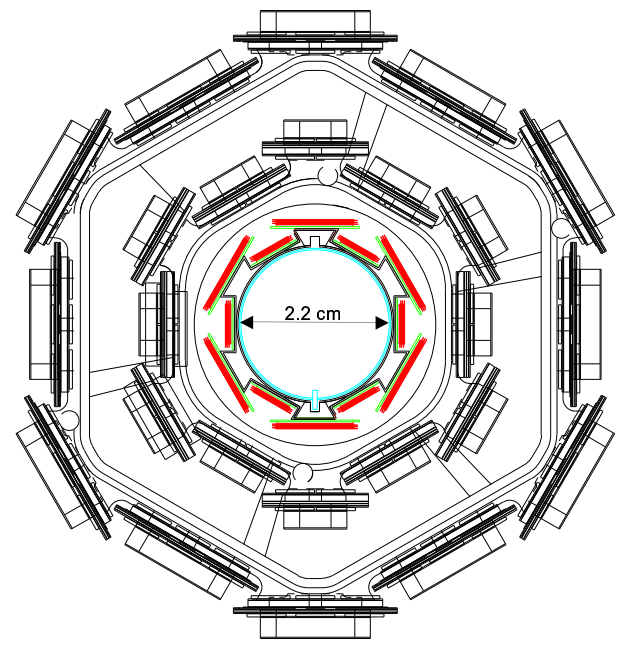

Figure 3.4: End view of the L00 tracker, shown surrounded by the first two layers of the SVX II. 
two different widths, 8.4 and $14.6 \mathrm{~mm}$ wide, and are interleaved in a 12-sided pattern as shown in Fig. 3.4. The inner/outer wafers are at a distance of $1.35 / 1.65 \mathrm{~cm}$ from the beamline. The wafers are all $7.84 \mathrm{~cm}$ long, but they are bonded together in pairs so that electrically they form $15.7 \mathrm{~cm}$ long detector elements. The entire length of the L00 detector is $0.9 \mathrm{~m}$.

Each of the wafers consist of p-doped strips implanted on an n-doped substrate. The strips have a pitch of $25 \mu \mathrm{m}$ and width of $8 \mu \mathrm{m}$, with the strip readout done through AC coupling to an insulated conductor above the doped signal strip. The readout pitch is actually $50 \mu \mathrm{m}$, since only alternating strips are used. The L00 has 13,824 individual readout channels.

Moving outward radially, the next five silicon layers comprise SVX II detector. The SVX II silicon wafers are arranged in ladders that are four wafers long, with two of the ladders on either side wire-bonded together so that electrically they form one detector element. The readout electronics for each of these 'half-ladders' are mounted directly to the silicon surface at either end of the ladder. The ladders are each $29 \mathrm{~cm}$ long and are arranged in a barrel structure as shown in Fig. 3.5.

The SVX II silicon wafers are all double sided. The bulk material is nearly pure silicon, although it is originally ${ }^{1}$ slightly n-doped. They all have p-doped strips on one side running in the axial direction. These strips are spaced between 60-65 microns apart and have widths of 14-15 microns, depending on the layer. The other side of the silicon wafer has n-doped strips that are either running at a $90^{\circ}$ angle, or a small stereo angle relative to the axial direction. The pattern for the five layers, running from the innermost to the outermost, is $\left(90^{0}, 90^{0},-1.2^{0}, 90^{0}, 1.2^{0}\right)$. The spacing of the stereo strips for each layer is $(141 \mu \mathrm{m}, 125.5 \mu \mathrm{m}, 60 \mu \mathrm{m}, 141 \mu \mathrm{m}, 65 \mu \mathrm{m})$, and the widths are $20 \mu \mathrm{m}$ for the $90^{\circ}$ strips and $15 \mu \mathrm{m}$ for the small angle stereo strips. All of the SVX II channels use a capacitive readout similar to that of the L00, with the $90^{\circ}$ layers having an additional layer of insulator and readout strips to carry the signal to the ends. The SVX II has a total of 405,504 readout channels.

The ISL is located between the inner silicon detectors and the drift chamber.

\footnotetext{
${ }^{1}$ I say originally because over time the radiation from the beam will in fact make the bulk material become slightly p-doped.
} 


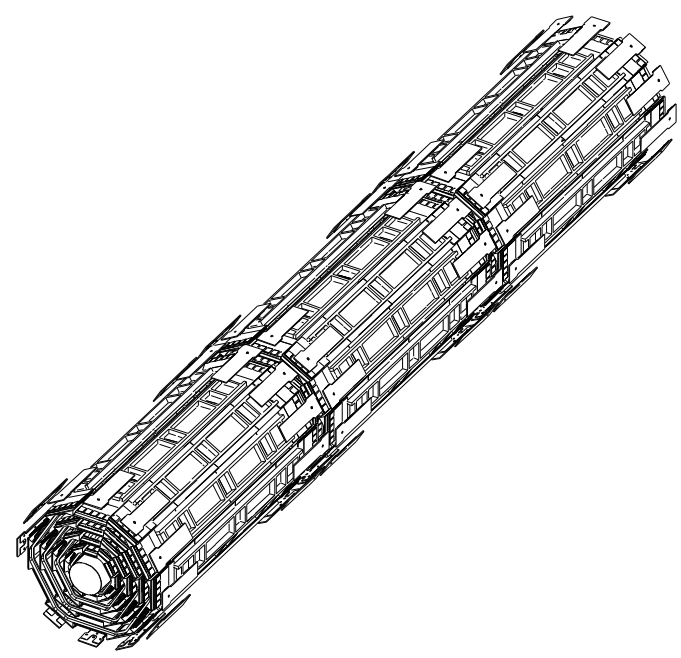

Figure 3.5: Diagram showing the three barrel structure of the SVX II.

The same as for the SVX II, the ISL sensors are double sided and use a capacitive readout. The strip pitch on both sides of the ISL sensors is $112 \mu \mathrm{m}$. Pitch adapters are used to bring signals to the more tightly spaced readout electronics (which are pitched to match the SVX II). The strips for an ISL wafer are in the axial direction on one side, and are at a $1.2^{0}$ small angle stereo direction on the other. Whether the stereo strips correspond to the $\mathrm{n}$ or $\mathrm{p}$ side varies for different wafers, depending on the manufacturer $^{2}$. ISL ladders are made up of two half-ladders which are in turn made of three silicon wafers. These are then constructed into a barrels, with the readout being done at the ladder ends. There is more space available than in the SVX II, allowing some of the ladders to be overlapped in $z$. The spatial distribution of the ISL barrels can be seen in Fig. 3.6. The central barrel ladders have staggered radii of $22.6 \mathrm{~cm}$ and $23.1 \mathrm{~cm}$. The forward barrel ladders consist of two layers. The inner forward layer is staggered at radii of $19.7 \mathrm{~cm}$ and $20.28 \mathrm{~cm}$, and the outer forward layer is staggered at radii of $28.6 \mathrm{~cm}$ and $29.0 \mathrm{~cm}$. The reason that the forward barrels have one more layer than the central barrel is to increase tracking acceptance in the $\eta$ region where COT efficiency drops off[19].

\footnotetext{
${ }^{2}$ The stereo strips are on the $\mathrm{n}$ side for sensors made by Micron, and on the $\mathrm{p}$ side for sensors made by Hamamatsu.
} 

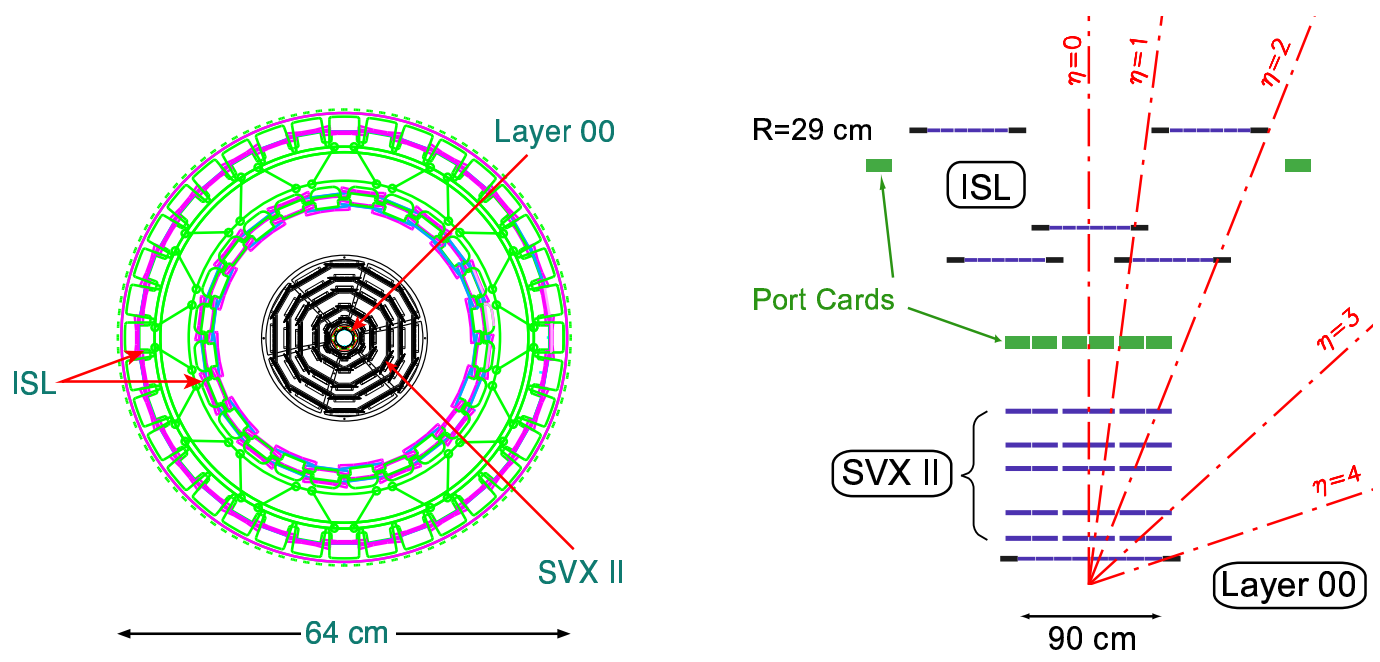

Figure 3.6: Left: End view of the CDF Run II silicon system; Right: Side view of half of the CDF Run II silicon tracking system. Note that the scale of the z-coordinate has been highly compressed in this figure.

\subsubsection{Central Outer Tracker (COT)}

The COT is a cylindrical drift chamber located at a radius of $43.4 \mathrm{~cm}$ to $132.3 \mathrm{~cm}$ from the beamline, surrounding the silicon tracking system. It is $310 \mathrm{~cm}$ long and covers the region of $|\eta|<1.1$. It is comprised of 96 radial sense wire layers organized into 8 "superlayers" of 12 wires each. Superlayers 1, 3, 5 and 7 are oriented at a stereo angle of $\pm 3^{0}$ relative to the beamline allowing the COT to resolve some $3 \mathrm{D}$ tracking (superlayers 0, 2, 4 and 8 are parallel to the beamline). Each superlayer is further divided in $\phi$ into "super cells". A super cell consists of one wire plane and one field plane on either side. The wire plane in each cell contains the 12 sense wires, 13 potential wires, and 4 field shaping wires Fig. 3.7 shows the nominal cell layout for superlayer 2. Cells are given a tilt angle of $35^{0}$ with respect to the $\hat{r}$ directions. This allows the left-right signal ambiguity to be resolved during track reconstruction.

A critical design element of the COT is that the drift time must be kept lower than the time spacing of the bunch crossings in the accelerator. In the present 36 bunch mode of the accelerator the bunches cross every 396ns. A mixture of Argon/Ethane (50:50) is used in the COT and has a maximum drift time of $177 \mathrm{~ns}$ over the $0.88 \mathrm{~cm}$ 


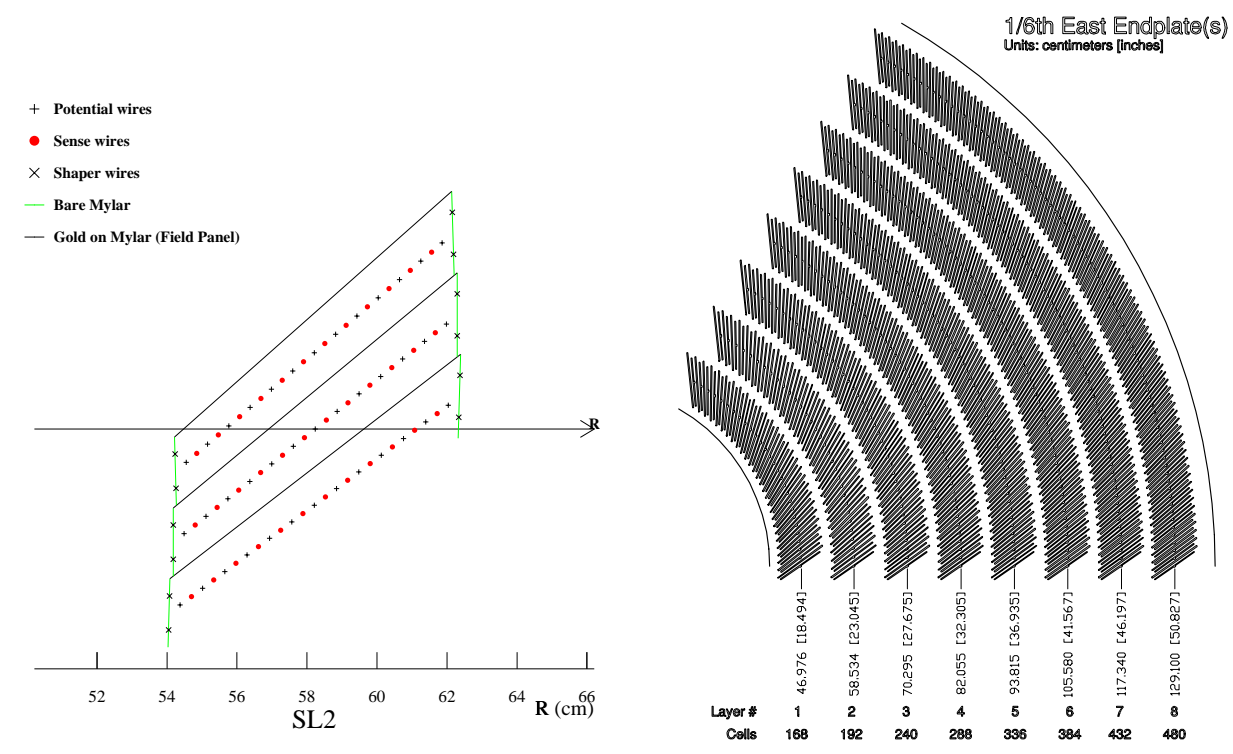

Figure 3.7: Nominal cell layout for superlayer 2, and arrangement of cells on the COT endplate.

maximum drift distance.

While the time of arrival of a signal pulse is used to determine the position of a tracked particle, the pulse width can be used to measure the amount of ionization caused by the particle. This is directly related to the amount of energy transfered by the particle to the ionizing material per distance traveled in the material $(\mathrm{dE} / \mathrm{dx})$. Since $\mathrm{dE} / \mathrm{dx}$ is determined by the velocity of the particle-and independent of it's mass-this information can be used to aid in identifying the tracked particle. A more detailed description of the COT can be found in reference [20]

\subsection{Calorimeter Systems}

The CDF calorimeter systems are located outside of the solenoid and surround the tracking region. They are separated into two main physical systems: central calorimeters which are configured cylindrically around the beam line, and plug calorimeters which are located forward of the tracking region as shown in Fig. 3.8. Each of these systems is comprised of an inner electromagnetic calorimeter and an outer hadronic 


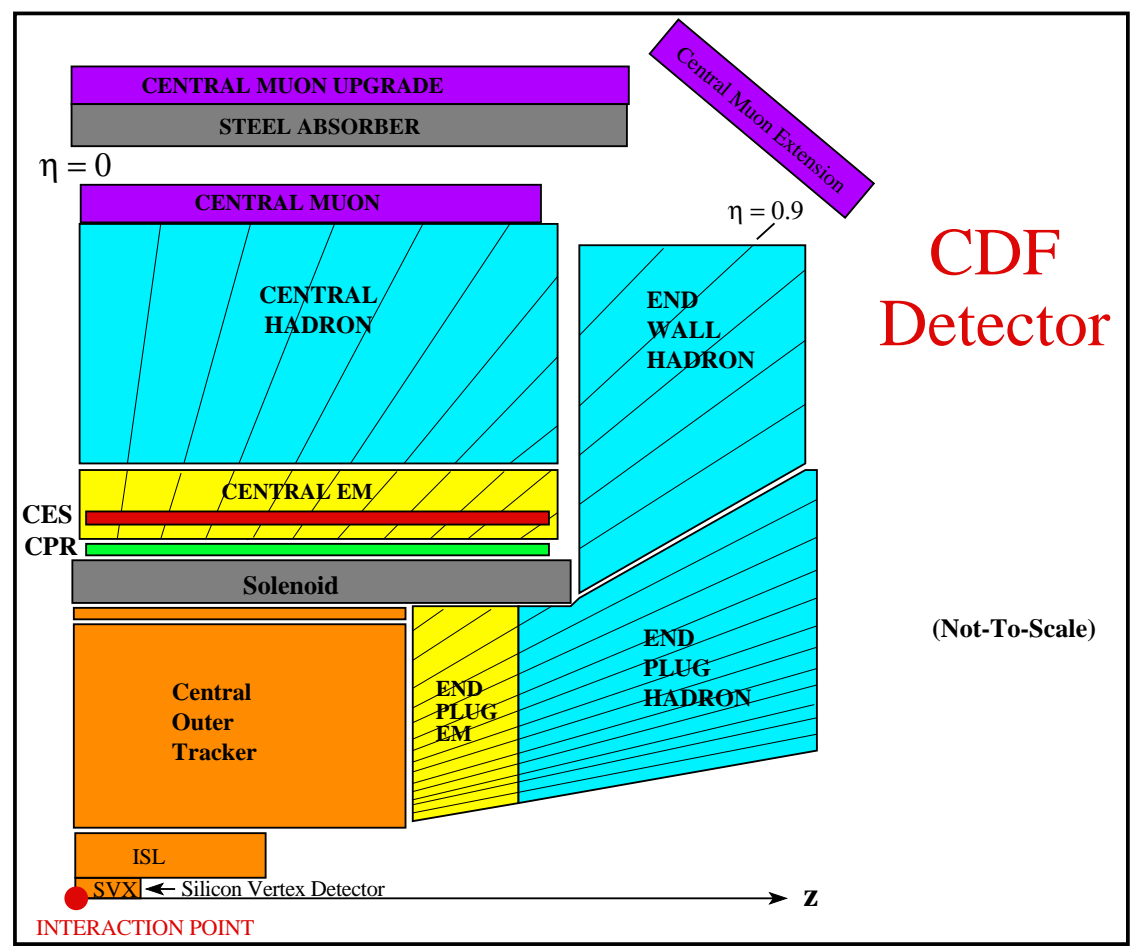

Figure 3.8: A lateral view of one quarter of the CDF detector showing the orientation of the calorimeter and central muon systems.

calorimeter. Both types are sampling calorimeters consisting of alternating layers of scintillator and absorber (made of lead and iron for the electromagnetic and hadronic calorimeters, respectively). There is also an end-wall hadronic calorimeter that covers the gap between the central and plug hadronic calorimeter systems. The calorimeters are segmented in both azimuth and pseudorapidity to form a projective tower geometry, allowing calorimeter data to be matched up with tracks/jets found by the tracking system. The calorimeter coverage runs over the region $|\eta|<3.64$ and the full $2 \pi$ azimuthally. Additionally, drift chambers are embedded in the electromagnetic calorimeters to provide precise position and profile information of the showers near their maximum. There are also similar preshower detectors located between the solenoid and the EM calorimeters to aid in track/tower matching. 


\subsection{Muon Detectors}

The muon detectors are made up of scintillators and drift chambers located at the exterior of the other detector systems. They are comprised of four similar detector systems distinguished by their physical configurations. The coverage of the various muon detectors is shown in Fig. 3.10. The Central Muon Detector (CMU) covers the region $|\eta|<0.6$ and is embedded in the outer edge of the central calorimeter wedges. The Central Muon Upgrade (CMP) also covers the region $|\eta|<0.6$, but contains two feet of steel between it and the CMU to reduce background from non-muons. Fig. 3.9 shows the geometry of muon counters in the central region.

The Central Muon Extension (CMX) consists of arches arranged at each end of the central detector, extending in polar angle from $42^{0}$ to $55^{0}$. These slightly overlap the central muon systems $\eta$ coverage, extending the total $|\eta|$ coverage out to 1.0 . No additional steel absorber was added for this detector; however the large angle through the hadron calorimeter, magnet yoke, and steel of the detector end supports does provide significant absorbing material. The azimuthal coverage of the CMX has a $30^{\circ}$ gap at the top. A $90^{\circ}$ azimuthal gap at the bottom of the detector, where the conical sections are interrupted by the collision hall floor, has been filled by a fan-shaped CMX section inserted into a gap between this section of the floor and the bottom of the magnet yoke.

The Intermediate Muon System (IMU) is a barrel of CMP-like chambers surrounding the beamline on either side of the detector. There are also pinwheels of counters on the endplate and inside the IMU barrel (between the toroids). It can trigger on muons up to an $|\eta|$ of 1.5, and can be used in conjunction with the tracking to identify muons up to an $|\eta|$ of $2.0[17]$. 


\subsection{Trigger Systems}

\subsubsection{Overview}

The collision rate at CDF for RunII is $2.5 \mathrm{MHz}$, while the maximum rate at which events can be recorded on tape is only about $50 \mathrm{~Hz}$. A trigger must be implemented to extract the most interesting physics events for recording, discarding the rest. The high event rate necessitates an extremely fast decision time for the trigger systems. To accomplish this CDF uses a three level architecture, with each level providing enough rate reduction to allow the next level sufficient time to process its events.

A functional diagram of the CDF RunII trigger system is show in Fig. 3.11. At Level-1 the data from the detector is stored in a pipeline with buffering for 42 beam crossings $^{3}$. Simultaneously, data from the central outer tracker, calorimeters, and muon detectors are sent to the Level-1 trigger system which uses custom hardware to find physics objects (tracks, jets, etc.) using this subset of the detector information. If the event is found to be sufficiently interesting it is then held in one of four Level-2 buffers for further processing by the Level-2 trigger hardware. The accept rate at Level-1 is $40 \mathrm{kHz}$, about $1.5 \%$ of the total number of events. The Level-2 system uses custom hardware to do limited event reconstruction, combining the Level-1 trigger information with data from the SVX and the calorimeter shower max detectors. This allows for the application of more sophisticated trigger cuts, reducing the maximum accept rate to $300 \mathrm{~Hz}$ at Level-2. A Level-2 accept initiates full detector readout for the event. The data are then sent to the Level-3 processor farm where full reconstruction is done on each event before a final trigger decision is made and the event is written to permanent storage.

\subsubsection{Level-1 Trigger}

A block diagram of the CDF RunII trigger system is shown in Fig. 3.12. The Level-1 decision is made using data only from the central outer tracker, calorimeter,

\footnotetext{
${ }^{3}$ At $132 \mathrm{~ns}$ between beam crossings. In the $396 \mathrm{~ns}$ mode at which the Tevatron is currently running the buffers for the two intermediate clock cycles are left empty and automatically rejected by the trigger. This makes for an effective pipeline of 14 beam crossings in 396ns mode.
} 


\section{Dataflow of CDF "Deadtimeless" Trigger and DAQ}

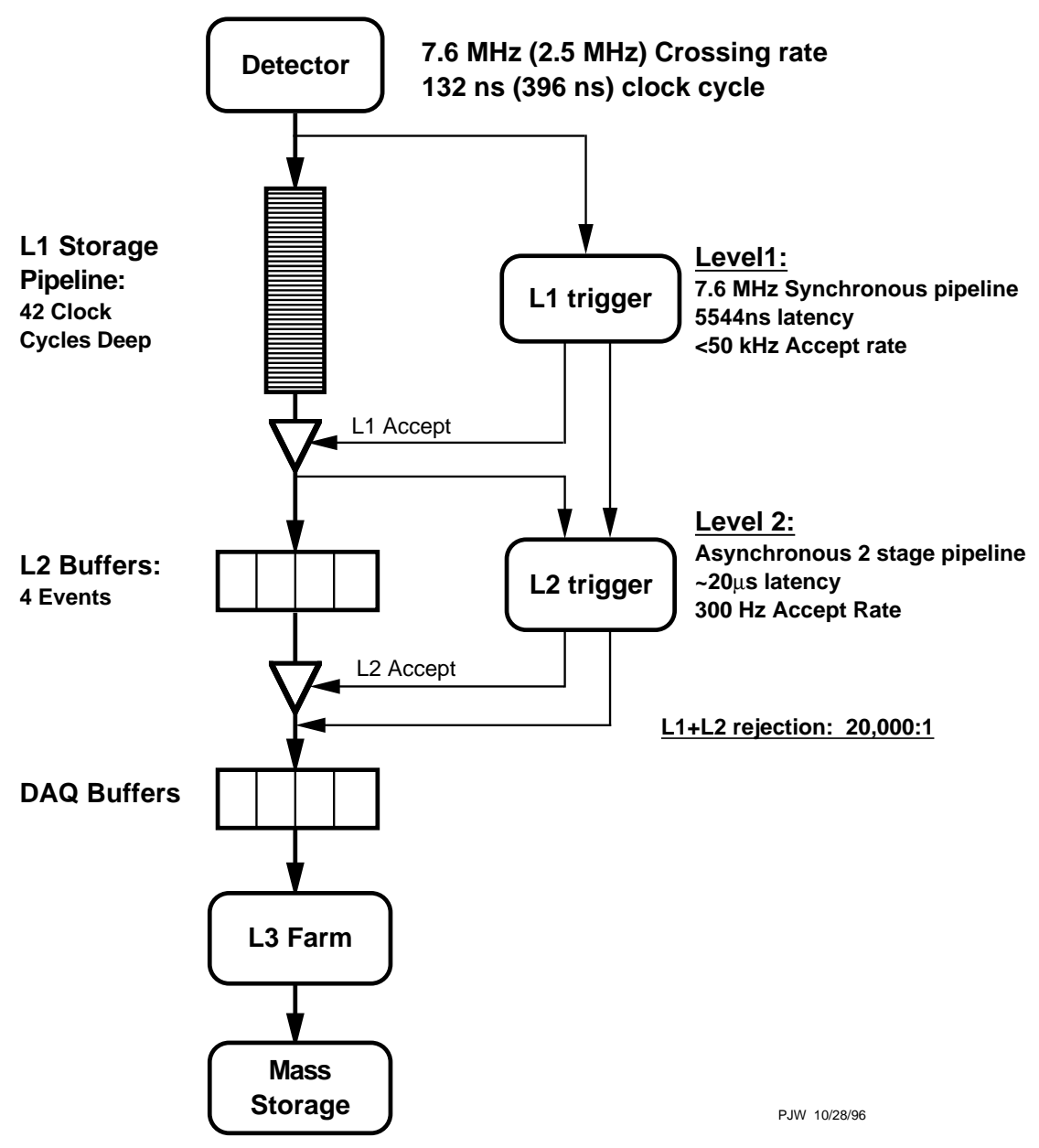

Figure 3.11: A functional diagram of the CDF run-II trigger system. 
and muon detector systems. Track finding is now done at Level-1. This means tracks can be matched to clusters in the electromagnetic calorimeter and to muon stubs in the muon detectors allowing, respectively, for electron and muon identification at Level-1. This is a significant improvement over the Run-I Level-1 trigger.

In the first step of Level-1 processing, data from the four axial superlayers of the central outer tracker is sent to the eXtremely Fast Tracker (XFT). The XFT is a highly parallel piece of custom hardware that processes the data from each bunch crossing. It is capable of finding tracks with a minimum transverse momentum of $1.5 \mathrm{GeV} / \mathrm{c}$ with an efficiency greater than $96 \%$ (for a single hit COT efficiency of $92 \%)$. It is also capable of finding the track transverse momentum with a resolution of $\Delta P_{T} / P_{T}^{2}<2 \%$ and the track azimuthal angle to better than 6 mrad.

After the tracks have been reconstructed by the XFT they are sent to the Extrapolation Unit(XTRP). The XTRP extrapolates the tracks from the COT out to the calorimeter and muon detector systems using look-up tables. It then passes the track information to each of the Level-1 subprocessors-L1CAL, L1MUON, and L1TRACK. The XTRP also contains a pipeline buffer to hold track information pending a Level-1 accept. Upon receiving a Level-1 accept for an event it transfers the track information to the Level-2 buffer, Level-2 processor, and the Silicon Vertex Tracker (to be described below).

At this point the Level-1 processing is done in three parallel synchronous streams

- L1CAL The Level-1 calorimeter hardware triggers on objects like electrons, photons, and jets, and on global information such as total transverse energy $\left(\Sigma E_{T}\right)$, and missing transverse energy $\left(\mathscr{F}_{T}\right)$. The object triggers are applied by requiring individual calorimeter tower signals to be above a specified threshold, while global triggers apply thresholds after summing the energy from all towers. Triggers can also be applied which require multiple objects above a certain prescribed threshold.

- L1MUON The Level-1 muon trigger finds single and dimuon objects for the Level-1 trigger decision. It does this by matching tracks from the XTRP to 
muon "primitives" which have been reconstructed from the muon detector data in the MUON PRIM hardware.

- L1TRACKS The Level-1 Track Trigger is an adjunct to the XTRP, and resides in the same physical crate. It makes a trigger decision based solely on the track information from the XTRP. For example, events with an extremely high transverse momentum track, or events with multiple tracks above some transverse momentum threshold can be selected.

After the event has been analyzed by the Level-1 subprocessors their various single-bit trigger signals are sent to the Global Level-1 hardware which makes the final Level-1 trigger decision. It does this by taking AND/OR combinations of the various bits from the different subprocessors. The Global Level-1 hardware is also capable of dynamically prescaling (rate limiting) any of the Level-1 triggers separately, if desired.

\subsubsection{Level-2 Trigger}

The Global Level-2 Trigger hardware uses input from several asynchronously running subsystems to make its decision. The processing for Level-2 begins after an event is written into the Level-2 buffer. While the data in one of the buffers is still being analyzed that buffer cannot be used to hold new events. If all four Level-2 buffers are simultaneously filled deadtime will occur. To keep the deadtime below an acceptable level the Level-2 decision time must be less than about $80 \%$ of the average time between Level-1 accepts. With the Level-1 trigger rate at $50 \mathrm{kHz}$ this would require the Level-2 trigger to make a decision in less than $16 \mu \mathrm{s}$. The minimum time in which the hardware can process the data is only $20 \mu s$, however, so a two tier pipeline for the data in Level-2 had to be implemented. The data is collected from the various subsystems in the first $10 \mu \mathrm{s}$, and analyzed during the second $10 \mu \mathrm{s}$. While the $20 \mu \mathrm{s}$ latency remains, one event can now be loaded into memory while the previous event is still being analyzed.

The Level-2 decision is made using all of the previous Level-1 information, but at a 


\section{RUN II TRIGGER SYSTEM}

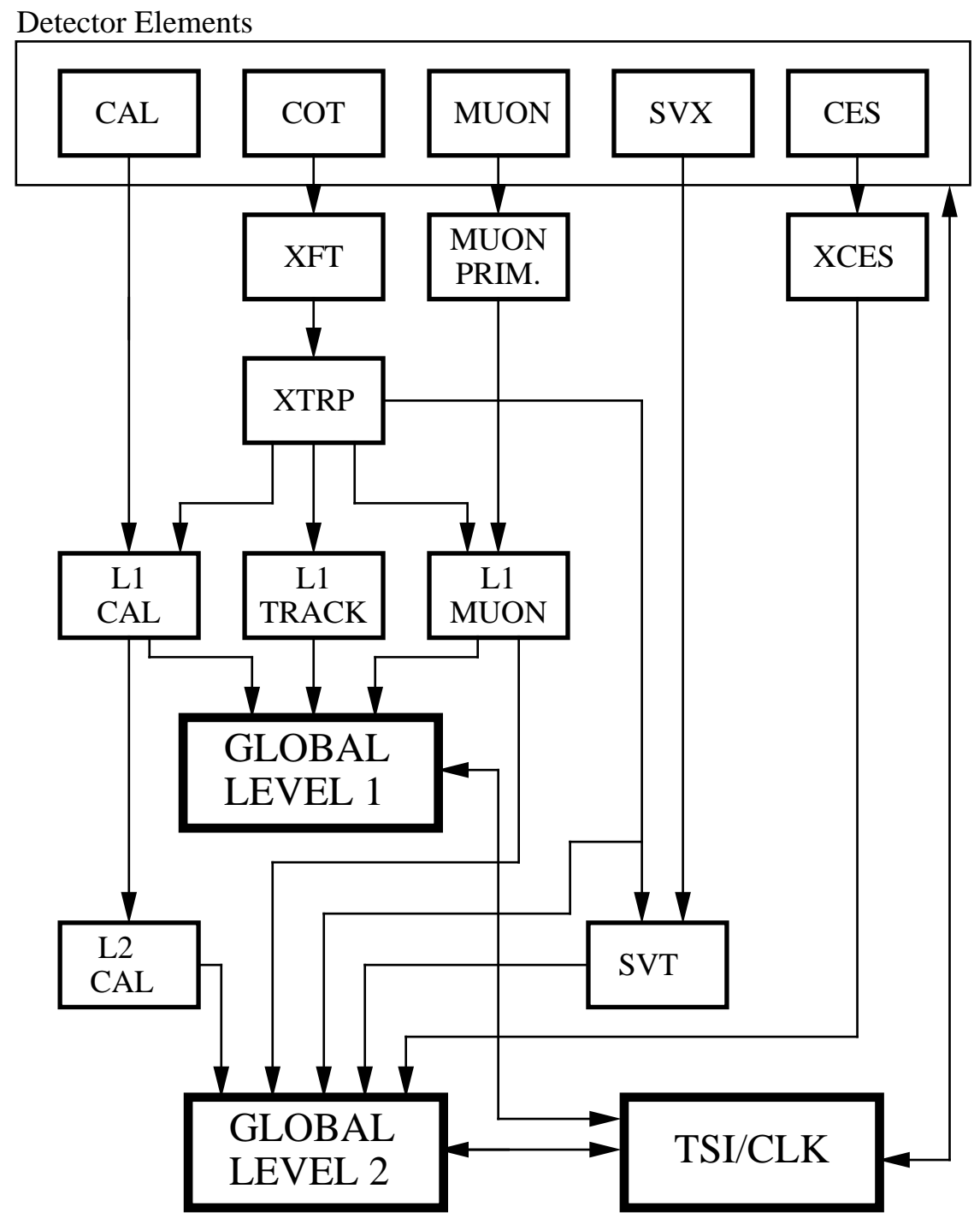

PJW 9/23/96

Figure 3.12: A block diagram of the CDF run-II trigger system. 
higher precision. The momentum resolution of the tracks is improved, a finer angular track matching to the muon primitives is done, and jet clusters are reconstructed in the central calorimeter data. Level-2 also uses additional information from the silicon vertex detector (SVX) and the shower maximum detectors of the electromagnetic calorimeters (CES).

- SVT The Silicon Vertex Tracker combines SVX-II data with the tracks from the XTRP and reconstructs 2-D tracks with great speed and with an accuracy comparable to a full offline analysis. It can precisely measure the impact parameter of tracks, and is the first trigger in a hadronic experiment capable of cutting on this quantity. Hadrons containing a b-quark have relatively long lifetimes in particle terms (on the order of $10^{-12} \mathrm{~s}$ ). Those generated at CDF can have enough momentum to travel a few millimeters before decaying, with the subsequent daughter particles having tracks with large impact parameters. Being able to cut on the track impact parameter therefore allows for a substantial increase in the the physics reach of the CDF detector since it can trigger on data rich in b quark content.

The SVT design is concentrated on speedy calculation due to the short decision time available at Level-2. A schematic of the SVT architecture is shown in Fig. 3.13. After every Level-1 trigger the SVX signal is digitized and read out the data signal is then split and simultaneously sent to the SVT. In the SVT, the SVX data are first run through a Hit Finder. There is one Hit Finder board for each of the 42 SVX wedges. The Hit Finders first perform pedestal subtraction and bad channel suppression on the SVX data. They then scan for clusters of hit strips, outputting the centroid of each cluster-the most probable track intersection point-to both the Associative Memory Sequencer(AMS) and the Hit Buffer. At the same time, tracks from the XFT are also sent to the AMS and Hit Buffer.

The AMS first converts the cluster centroids into coarser "superstrips" about $250 \mu \mathrm{m}$ wide. The superstrip width is adjustable and is a compromise between better pattern recognition and less required memory. The AMS then broadcasts 
the XFT tracks and coarse SVX clusters onto two Associative Memory (AM) boards. Each board contains 128 associative memory chips each of which in turn is programed with the patterns for 128 different possible roads (legitimate particle trajectories). The chips check to see if all of the components for any of its roads are present in the data. The AMS then passes the roads for any track candidates to the Hit Buffer. The hit buffer then collates the full outer-track and SVX cluster information for each road into track packets (sets of one outer track and four SVX hits) and sends the information on to the Track-Fitter farm. The farm contains 10 track fitter modules which fit the tracks using a linear approximation consisting of a set of scalar products. Their output is then sent out to the Global Level-2 processor which uses the information to apply the Level-2 trigger cuts.

- L2CAL The Level-2 Cluster Finder is used to reduce the jet trigger rate. In general, jets are not fully contained in a single calorimeter tower, so the Level-1 trigger thresholds must be set lower than the jet energy to be efficient. This makes the trigger rate too high, however, so at Level-2 contiguous regions of calorimeter towers with non-trivial energy are combined to form clusters, allowing a cut to be applied on the cluster's total transverse energy.

- XCES The shower maximum detectors located in the electromagnetic calorimeters are used to reduce the trigger rate for electrons and photons. Requiring a cluster above threshold in the shower max detector helps reduce background from single phototube discharges, and the improved resolution with respect to the calorimeter wedges helps reduce combinatoric background in the track matching.

All of the available Level-2 information (SVT, track information, muon information, XCES, and Cluster Finder) is passed to the Global Level-2 decision making hardware, which determines if the event passes the Level-2 trigger. A Level-2 accept initiates full detector readout for the event. 


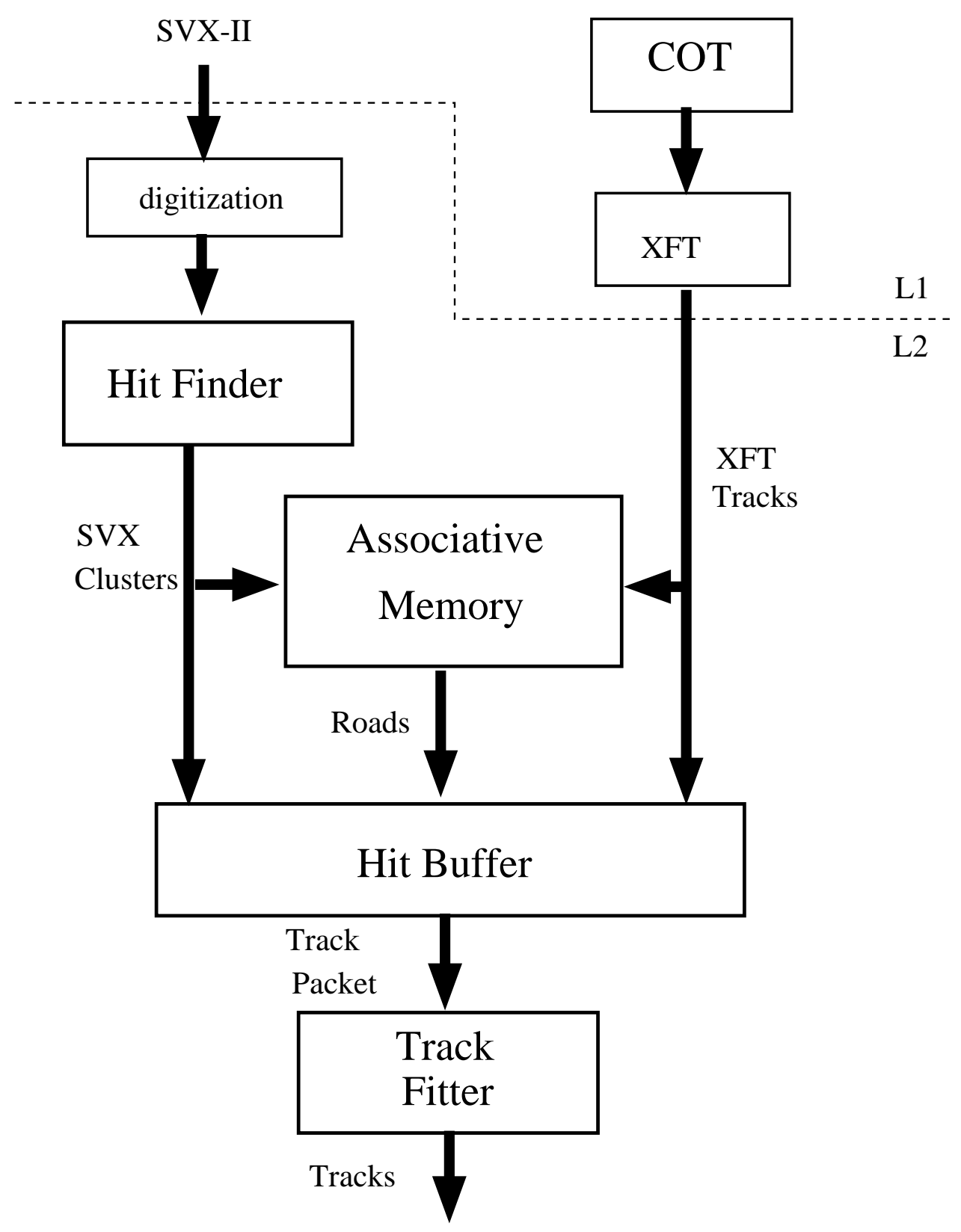

L2 Decision

Figure 3.13: Architecture of the SVT trigger. 


\subsubsection{Event Builder and Level-3 Trigger}

Since they must operate on such a short time scale, the Level-1 and Level-2 triggers use only a small, pre-defined subset of the entire event data to make their decision. Pending a possible Level-2 accept, the full event data is stored on several buffers located in front-end electronics throughout the detector. After a Level-2 accept the Event Builder retrieves all of the data fragments for the passed event and sends them via a high speed network to one of sixteen converter nodes.

Each converter node is connected to 14-18 processor nodes, forming a Level-3 "subfarm". A converter node assembles the event fragments into one data block formatted for analysis, and distributes it to one of its processor nodes. The converter nodes have multiple event buffers, and can receive one event while in the process of distributing another. The processor nodes are PCs running Level-3 algorithms that take advantage of the full detector information, this includes performing full 3-dimensional track reconstruction and tight matching of tracks to calorimeter and muon-system information[17, 21]. If an event passes the Level-3 trigger cuts it is then sent to the Consumer Server Logger(CSL). The CSL writes the event data to disk and distributes some small fraction of events to on-line consumer monitoring programs which verify data quality. 


\section{Chapter 4}

\section{Data Sample}

\subsection{Hadronic Data Sample}

After the Level 3 trigger selection, the raw data at CDF is processed on a PC farm using the CDF production software[22]. The software reconstructs high-level objects such as tracks, electrons, muons, and jets. The reconstructed events are then separated into different data sets based on which triggers they passed. This analysis uses the data from the hbot0h data set, the first half of the hbhd09 data set (run numbers 158821 - 161781) and the available hbot1i dataset as of July 3rd, 2003. Runs in common between the hbhd09 and hbot1i dataset were explicitly excluded (e.g. they were not run on twice). All of these h-series data come from a specific trigger called the Two-Track Hadronic Trigger. This trigger was designed to collect data with enhanced charm and bottom hadron content by using the fact that hadrons containing charm or bottom particles have relatively long lifetimes and can decay at a secondary vertex some distance away from the primary interaction point. This trigger was proposed well before the Run-II upgrade, and has been studied extensively using Run-I data and signal Monte Carlo [24].

A typical store in the Tevatron can last around 20 hours. During this time the luminosity steadily decreases, regularly finishing at around only $40 \%$ of its initial value. The trigger system was designed for operation at peak luminosity, so there is extra trigger bandwidth available at the end of a store. This extra bandwidth is 
filled by using multiple triggers with different $p_{T}$ thresholds and prescaling the lower threshold triggers (which have the higher trigger saturation). There are three versions of the Two-Track Hadronic Trigger stream: nominal, low- $p_{T}$, and $h i g h-p_{T}{ }^{1}[25]$. Data from all three of these trigger streams were used in this analysis. Typical definitions for each of these trigger streams are below, however the exact details of their operation can change slightly over time. Time dependent details for these trigger streams can be found in $[26]$.

\subsubsection{The Nominal Two-Track Trigger Stream}

The Level-1 trigger decision for the nominal two-track trigger path is made using XFT tracks. This stream is dynamically prescaled if the Level-1 accept rate is ever in excess of $20 \mathrm{kHz}$. The Level-1 selection cuts are:

- two XFT tracks of opposite charge

- 4 XFT layers per track

- $p_{t}>2.04 \mathrm{GeV} / \mathrm{c}$ for both tracks

- $0<\Delta \phi<135^{0}$

- scalar $\Sigma p_{T}$ of two tracks greater than $5.5 \mathrm{GeV} / \mathrm{c}$

The Level-2 trigger decision for the nominal two-track trigger path is made using SVT tracks. The selection cuts are:

- two SVT tracks of opposite charge

- $\operatorname{SVT} \chi^{2}<25$

- $p_{t}>2.0 \mathrm{GeV} / \mathrm{c}$ for both tracks

- scalar $\Sigma p_{T}$ of two tracks greater than $5.5 \mathrm{GeV} / \mathrm{c}$

\footnotetext{
${ }^{1}$ These triggers are often referred to as B_CHARM, B_CHARM_LOWPT, and B_CHARM_HIGHPT in the CDF vernacular.
} 
- $2^{0}<\Delta \phi<135^{0}$

- $120 \mu m<\left|d_{0}\right|<1 m m$

- $L_{x y}>200 \mu m$ (transverse decay length)

At Level-3 the SVT tracks are matched to COT tracks by requiring proximity in curvature and $\phi_{0}$. The cuts are performed on the COT track parameters, except for the impact parameter $\left(d_{0}\right)$ which is inherited from the matched SVT track. The Level-3 selection criteria are:

- two tracks of opposite charge

- $|\eta|<1.2$ for both tracks

- $p_{t}>2.0 \mathrm{GeV} / \mathrm{c}$ for both tracks

- scalar $\Sigma p_{T}$ of two tracks greater than $5.5 \mathrm{GeV} / \mathrm{c}$

- $2^{0}<\Delta \phi<135^{0}$

- $120 \mu m<\left|d_{0}\right|<1 m m$

- $\left|\Delta z_{0}\right|<5 \mathrm{~cm}$ between the two tracks

- $L_{x y}>200 \mu m$

\subsubsection{The $L o w-p_{T}$ Two-Track Trigger Stream}

The $l o w-p_{T}$ two-track trigger path is used during lower luminosity periods to fill any remaining bandwidth available to the bottom triggers. This stream is only enabled when the Level-1 accept rate is below $16 \mathrm{kHz}$. Its trigger criteria are looser than the nominal path described above, allowing ample events to be accepted. It is then dynamically prescaled to an accept rate commensurate with the remaining bandwidth.

The Level-1 trigger decision for this path is made using XFT tracks. The Level-1 selection cuts are: 
- two XFT tracks (no opposite charge requirement)

- 4 XFT layers per track

- $p_{t}>2.04 \mathrm{GeV} / \mathrm{c}$ for both tracks

- $\Delta \phi<90^{0}$

- no scalar $\Sigma p_{T}$ requirement

The Level-2 trigger decision for the low- $p_{T}$ two-track trigger path is made using SVT tracks. The selection cuts are:

- two SVT tracks

- $\operatorname{SVT} \chi^{2}<25$

- $p_{t}>2.0 \mathrm{GeV} / \mathrm{c}$ for both tracks

- $\Delta \phi<90^{0}$

- $120 \mu m<\left|d_{0}\right|<1 m m$

- $L_{x y}>200 \mu m$

At Level-3 the SVT tracks are matched to COT tracks by requiring proximity in curvature and $\phi_{0}$. The cuts are performed on the COT track parameters, except for the impact parameter $\left(d_{0}\right)$ which is inherited from the matched SVT track. The Level-3 selection criteria are:

- two tracks in the event

- $p_{t}>2.0 \mathrm{GeV} / \mathrm{c}$ for both tracks

- scalar $\Sigma p_{T}$ of two tracks greater than $4.0 \mathrm{GeV} / \mathrm{c}$

- $2^{0}<\Delta \phi<90^{0}$

- $120 \mu m<\left|d_{0}\right|<1 \mathrm{~mm}$

- $\left|\Delta z_{0}\right|<5 \mathrm{~cm}$ 


\subsubsection{The $H i g h-p_{T}$ Two-Track Trigger Stream}

The high- $p_{T}$ two-track trigger path is essentially the same as the nominal trigger path, except that the $p_{T}$ and $\Sigma p_{T}$ thresholds are set higher to lower the accept rate. It is intended as a low yield trigger path that would never be prescaled during data taking. This trigger path could then be used in a straight-forward manner for crosssection measurements. The Level-1 trigger decision for the $h i g h-p_{T}$ two-track trigger path is made using XFT tracks. The Level-1 selection cuts are:

- two XFT tracks of opposite charge

- 4 XFT layers per track

- $p_{t}>2.46$ (changed from initial value of 2.23 ) $\mathrm{GeV} / \mathrm{c}$ for both tracks

- scalar $\Sigma p_{T}$ of the two tracks is greater than 6.5 (changed from initial value of 6.0) $\mathrm{GeV} / \mathrm{c}$

- $0<\Delta \phi<135^{0}$

The Level-2 trigger decision for the $h i g h-p_{t}$ two-track trigger path is made using SVT tracks. The Level-2 selection cuts are:

- two SVT tracks of opposite charge

- $\operatorname{SVT} \chi^{2}<25$

- $p_{t}>2.5$ (changed from initial value of 2.25 ) $\mathrm{GeV} / \mathrm{c}$ for both tracks

- scalar $\Sigma p_{T}$ of two tracks greater than 6.5 (changed from initial value of 6.0 ) $\mathrm{GeV} / \mathrm{c}$

- $2^{0}<\Delta \phi<135^{0}$

- $120 \mu m<\left|d_{0}\right|<1 m m$

- $L_{x y}>200 \mu m$ 
At Level-3 the SVT tracks are matched to COT tracks by requiring proximity in curvature and $\phi_{0}$. The cuts are performed on the COT track parameters, except for the impact parameter $\left(d_{0}\right)$ which is inherited from the matched SVT track. The Level-3 selection criteria are:

- two tracks of opposite charge

- $|\eta|<1.2$ for both tracks

- $p_{t}>2.0 \mathrm{GeV} / \mathrm{c}$ for both tracks

- scalar $\Sigma p_{T}$ of two tracks greater than $5.5 \mathrm{GeV} / \mathrm{c}$

- $2^{0}<\Delta \phi<90^{0}$

- $120 \mu m<\left|d_{0}\right|<1 m m$

- $\left|\Delta z_{0}\right|<5 \mathrm{~cm}$

- $L_{x y}>200 \mu m$

\subsubsection{Good Run Selection}

Not every run taken at CDF is suitable for use in physics analysis. Problems may occur either in the hardware or software that make the data for a particular run unusable. In this analysis only "Good runs" have been used as defined by the Bvalidation group[27], a subgroup of the CDF validation group concerned specifically with verifying the integrity of data used in B-analysis. Information about the current status of the data acquisition system, triggers and rates, and beam conditions at CDF are recorded in a database on a run-by-run basis[28]. For a run to be considered good the following entries in the run-configuration table of the database must be true.

- RUNCONTROL_STATUS - This bit is automatically set true by the Run Control software when a run lasts long enough for 100 million collisions, 10,000 Level-1 accepts, 1,000 Level-2 accepts, and at least $1 n b^{-1}$ of integrated live luminosity. 
- SHIFTCREW_STATUS - This bit is filled by the shift crew member responsible for running the data acquisition software at the end of every run.

- OFFLINE_STATUS - This bit is filled by the offline monitor.

- CLC_STATUS - this bit is set to true if the CLC high voltage was on during the turn and the consumer plots of luminosity and beam conditions are normal.

- L1T_STATUS and L2T_STATUS - These bits are set true by the shift crew member responsible for verifying the integrity of the data after verifying that Trigger Monitoring plots are normal.

- L3T_STATUS - This bit is set to true if the SVX reformatter error is less than $1 \%$.

- SVX_STATUS - This bit is set to true if the SVX was powered on during the run.

- SVT_STATUS - This bit is set to true if the SVT online beam position subtraction was done correctly and the SVT occupancy is normal.

- COT_OFFLINE - This bit is set after the data has been examined offline by experts. The criteria for setting it to true are that there were fewer than $1 \%$ bad COT channels during the run and that the integrated live luminosity was at least $10 n b^{-1}$.

- SVT_OFFLINE - This bit is set after the data has been examined offline by experts. The criteria for setting it to true is that the $D^{0}$ and $D^{*+}$ yields are within the expected ranges ${ }^{2}$. For the later part of the data used in this analysis the SVT_OFFLINE bit was not used (it had not yet been set by the experts), and the data quality had to be verified explicitly as described in Section 4.1.5.

After exclusion of "bad" runs, the total integrated luminosity used in this analysis is about $115 p b^{-1}$.

\footnotetext{
${ }^{2}$ These particles decay at secondary vertices (are capable of firing the two track trigger) and are produced at a high enough rate to provide meaningful statistics on a run-by-run basis.
} 


\subsubsection{Verification of Data Quality}

To verify the new data (data taken after the January 2003 shutdown) quality the yields for $D_{s} \rightarrow \phi \pi$ and $D^{ \pm} \rightarrow \phi \pi$ were plotted as functions of run ranges. The results are shown in Fig. 4.1.

The run range 155130-155364 is where the shutdown takes place. The region of increasing yield during the period right after the shutdown is due to increased coverage in the SVX. The decrease in yields after run number 161379 can be attributed to a shift in the beam line of $\sim 0.5 \mathrm{~mm}$ further from center which affected the Level 1 two track trigger efficiency due to significant bias in the XFT track parameters. Accounting for these affects the $D_{s} / D^{ \pm}$yields are consistent with those expected.

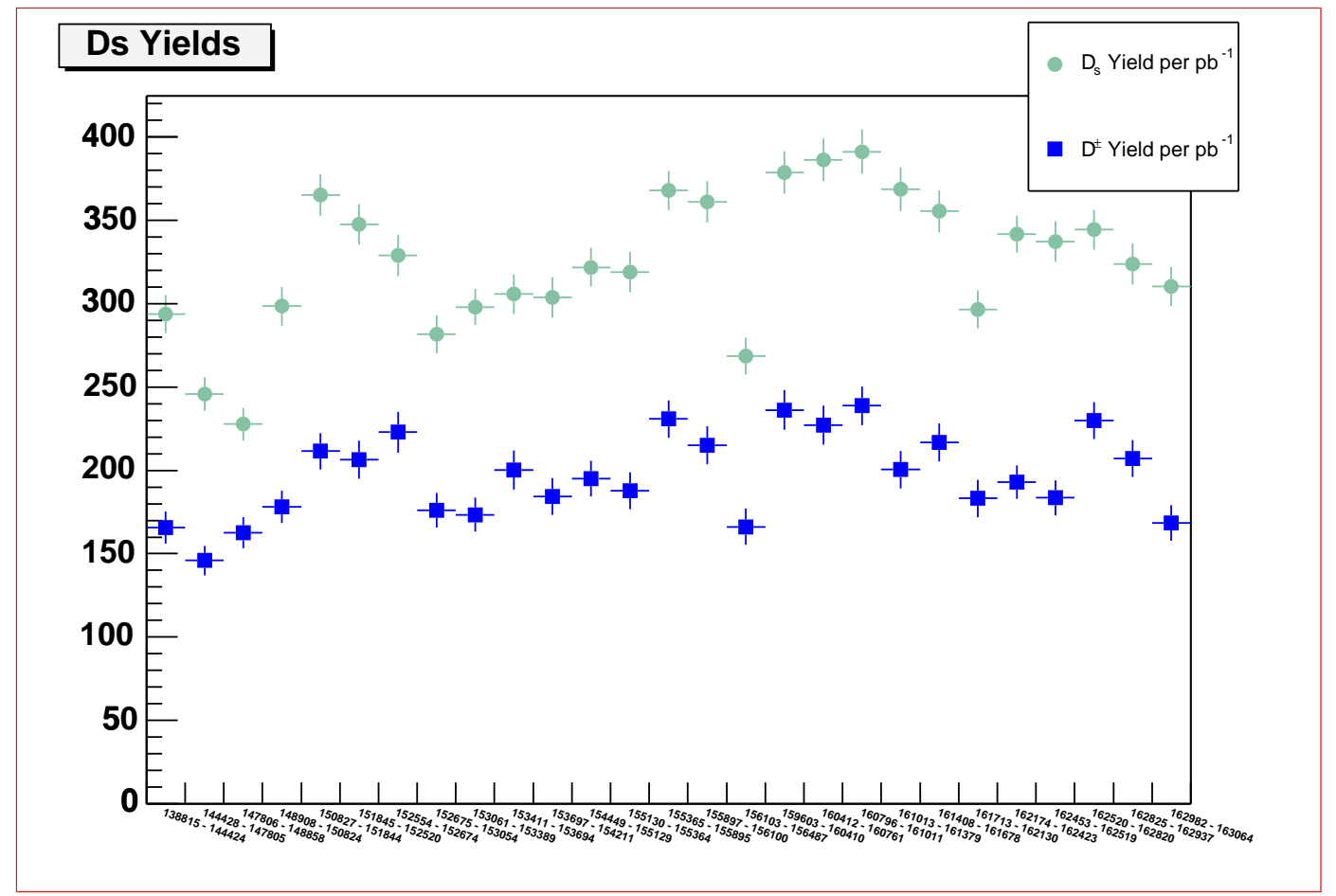

Figure 4.1: Plot of $D_{s} / D^{ \pm}$yield vs. run ranges. 


\subsection{Monte Carlo Data Sample}

Monte Carlo samples were used in this analysis as a cross-check of the reconstruction efficiencies for the various decay channels and to gain an understanding of background distributions. In general, Monte Carlo samples are prepared in three distinct steps. First, a particle generator is used to simulate the initial b quark(s) production and their subsequent fragmentation into B-hadrons. Next, a decay package is used to simulate the decay of the B-hadrons into stable particles. Finally, simulations of the detector and trigger responses to these particles are done, generating "realistic" Monte Carlo data ${ }^{3}$.

\subsubsection{B Generator}

The $b \bar{b}$ Monte Carlo generator used for this analysis is the CDF software package known as "Bgenerator" $[29,30]$. It is capable of either generating single b quarks and fragmenting them into single B-hadrons, or of generating $b \bar{b}$ pairs and fragmenting the two quarks into two hadrons. In this analysis only single b quark events were generated. The input to Bgenerator for single b quark generation is a two dimensional histogram giving the proper correlations between the $p_{T}(b)$ and $\eta(b)$ spectra for the $\mathrm{b}$ quark. The $p_{T}(b)$ spectra used were calculated according to the method proposed by Nason, Dawson, and Ellis (commonly referred to as NDE) [31, 32] in the range $p_{t}(b)>$ $4.0 \mathrm{GeV} / \mathrm{c}$ and $|\eta|<1.3$. In these calculations, the parton distribution function used was the MRSD0 partition function of Martin, Roberts, and Stirling[33, 34], the mass of the bottom quark was set to $m_{b}=4.75 \mathrm{GeV} / \mathrm{c}$, the mass of the charm quark was set to $m_{c}=1.5 \mathrm{GeV} / \mathrm{c}$, and the renormalization energy scale was set to $\mu=\mu_{0}\left(=\sqrt{P_{T}^{2}+m_{b}^{2}}\right)$. All of these are the CDF B-group defaults. After a $\mathrm{b}$ quark is produced it is fragmented to a B-hadron according to the Peterson fragmentation function. The Peterson epsilon used was the CDF default value of $\epsilon_{b}=0.006$. In the generation, the b quark can be forced to fragment into a specific B-hadron. In the

\footnotetext{
${ }^{3}$ As opposed to a "parametric" model also commonly used at CDF that does not fully simulate the detector response, but can generate Monte Carlo data at a much faster rate. This analysis does not use parametric Monte Carlo data.
} 
case of this analysis they were all forced to fragment to $B^{ \pm}$.

\subsubsection{The QQ Decay Packages}

The QQ decay package[35] was originally designed for use at the CLEO highenergy physics experiment at Cornell University[36]. It has since been adapted for use at CDF where it is applied on Monte Carlo events to decay particles that contain bottom or charm quarks. The QQ decay file contains all known decay channels for B mesons and baryons. In this analysis the QQ package was used to force the decays into the specific channels under study, taking into account the helicities. To save CPU time, some cuts were applied at the particle level before running the full simulation on the event. These cuts were kept very loose so that they would have a negligible effect on the final results (events that failed here would also have failed the cuts applied later during trigger simulation). The cuts required two tracks in the event with:

- $|\eta|<1.4$

- $0.015<\Delta \phi<1.8 \mathrm{rad}$

- $p_{T}>1.5 \mathrm{GeV} / \mathrm{c}$

- $\Sigma p_{T}>4 \mathrm{GeV} / \mathrm{c}$

\subsubsection{Trigger and Detector Simulation}

The realistic simulation at CDF does a detailed detector simulation using the GEANT software package and trigger simulation using emulation software developed at CDF. The GEANT software package is a general purpose detector description and simulation tool which can describe the passage of elementary particles through matter[37, 38]. A detailed description of the CDF detector geometry has been defined, allowing the GEANT software to track simulated particles as they pass through the detector and then simulate the detector response. The simulated detector output is given in the same format as real data. The simulated data is then passed to the CDF trigger emulation software which simulates the CDF trigger systems, determining 
if the virtual event would have passed the trigger cuts. The realistic simulation can reproduce the details of real data very well, but it takes a considerable amount of CPU time-about $1 / 2$ a minute per event-largely due to the complex geometry description of the CDF detector.

The exact configuration of the detector and trigger systems does change over time (e.g. regions of the silicon detectors could become damaged or previously dead regions could be recovered). This effectively creates a time dependence on the trigger efficiency. The SVT-based triggers in particular are sensitive to changes in the silicon detectors and the beam position. The conditions for a specific data run can be simulated by providing the corresponding detector and trigger system configurations. In this analysis Monte Carlo events were generated using the detector and trigger conditions from eight different "representative" runs: 141508, 144578, 146920, 150799, 151513, 152747, 153618, and 155148. Further details on the Monte Carlo sample can be found in Section 5.4.

\subsection{Offline Track Reconstruction and Refitting}

\subsubsection{Offline Track Reconstruction}

Tracks for charged particles are reconstructed using data taken by the COT and Silicon tracking systems. Since the COT is at a larger radius, the track density is lower there than in the silicon detectors. The fact that the tracks are more isolated in the COT results in fewer combinatorics for the track reconstruction. This makes track reconstruction easier-and faster-in the COT than it is in the silicon detectors, so tracking is done there first. The first step is to translate the TDC readout from the COT into valid hit positions. Within each superlayer, these hits are then linked into segments using the same basic algorithm as in Run I[39]. After the segments have been found, two algorithms are used to join the segments into tracks.

The first algorithm performs "segment linking" as described in [40]. Segments in the outermost superlayer (SL8) are used to generate an initial set of rough "tracks", assuming no impact parameter. The expected $\phi$ position of each of these tracks in 
the next-to-last axial superlayer (SL6) is found, and segments there are linked to the original. A fit is then made to find the track parameters of this two-segment track. Segments from the other superlayers are then linked to this track sequentially. Segments from the axial layers are all attached first so that the track is initially only 2-dimensional, and the stereo layers are attached later to make a full 3-dimensional track.

The second COT tracking algorithm uses a "histogram linking" method. A segment position and the beam position are used to define a reference track. A histogram is then made using the positions in curvature space of the hits on the other superlayers. Hits along the track all populate the same bin in this histogram, identifying the $\operatorname{track}[41]$.

The two COT tracking algorithms are used in conjunction to attain the best performance and speed of reconstruction. Tracks that are made using only the COT data are commonly referred to as COT-only tracks.

In the silicon detector, charge is deposited on the readout strips when a charged particle passes through the bulk material. This charge can then be translated into hit positions. Initially, tracking is done in the silicon detectors using COT tracks as seeds. A "window" is defined in the region where a COT track intersects with the outermost layer of silicon, the size of the window being determined by the uncertainties on the track parameters. All silicon hits within the window are attached to the track one at a time, and a fit is performed in each case. The output of the fit is then used as a seed track to add hits from the next layer, and the process is repeated until all layers have been searched. Similar to the COT algorithm, $r-\phi$ hits are picked up first and the stereo hits are added later. This is known at CDF as the "Outside-In" silicon tracking algorithm[42]. In the end, it is possible to have multiple output tracks generated from a single COT seed track if it attaches different combinations of valid silicon hits. The best one is chosen based on the $\chi^{2}$ of the track fit and the number of attached silicon hits.

After the Outside-In tracking is complete, there are still some silicon hits left untracked, i.e. there are hits that were not picked up by any COT seed track. A standalone silicon tracking algorithm has been developed at CDF to do tracking on 
these hits[44]. It is mostly useful for tracking in the forward region not covered by the COT (the silicon detectors cover tracking out to $|\eta|<2$, whereas the COT covers tracking out to $|\eta|<1$ ), but it can also recover some of the inefficiencies of the COT/Outside-In tracking in the central region.

\subsubsection{Track Refitting}

The tracks described in the previous section are called CDF default tracks (defTracks). Some final manipulations must be made before they are ready for use in analysis.

The COT tracks were made without consideration of Multiple Coulomb Scattering (MCS) effects in the COT volume. While this does not affect the actual values of the track parameters, it does result in an underestimation of the associated errors. To rectify this, the elements of the track covariance matrix are rescaled according to the suggestions made in [43] for the 4.9.1hpt3 version of the CDF software.

As charged particles pass through the detector they deposit energy in the detector material. This energy is responsible for the ionization that takes place in the bulk silicon and COT gas, allowing the particles to be tracked. As the particle loses energy its momentum decreases, and the curvature of the track changes along the particle path. It is the particles' starting momenta that are required for the event reconstruction described in Section 5.2.3, so this energy loss in the material must be accounted for. The energy loss per unit length in a material, $\frac{d E}{d x}$, is dependent on which type of particle is being tracked. The default tracks at CDF are fit using a pion hypothesis. This analysis is concerned with the reconstruction of $B^{ \pm} \rightarrow \phi K^{ \pm}$ where the $\phi \rightarrow K^{+} K^{-}$, i.e. a reconstruction of the event using kaon tracks. To this end, the tracks used in this analysis must be refit under a kaon hypothesis.

The refit procedure is done using the recommendations made by the CDF B-group:

- Hits from ISL and LOO are not included in the fit. The alignment details of these two detectors are still under study.

- The refitting is done using the alignment table "100030 1 GOOD". 
- The fit was performed using the Kalman fitter provided by the Karlsruhe group and described in [44].

- The nominal magnetic field in the vertex fitter was rescaled as recommended in [45]. 


\section{Chapter 5}

\section{Physics Analysis}

\section{$5.1 \quad$ Overview}

In this analysis a search is made for the decay mode $B^{ \pm} \rightarrow \phi K^{ \pm}$where the $\phi \rightarrow K^{+} K^{-}$at CDF using the Two Track Hadronic Trigger data (described in Section 4.1), and the number of events is measured. The small yield for this decay mode makes direct determination of its branching ratio prohibitively difficult, so instead it is compared to a normalization mode, $B^{ \pm} \rightarrow J / \psi K^{ \pm}$where the $J / \psi \rightarrow \mu^{+} \mu^{-}$, and the ratio of the two branching ratios is calculated. The two decay modes are topologically similar, and the majority of systematic errors will therefore cancel.

The reconstruction efficiencies for the two decay modes are found using Monte Carlo events, excluding the isolation cut which cannot be effectively modeled by the Monte Carlo. The ratio of the isolation cut efficiencies for each mode is calculated using the $B^{ \pm} \rightarrow J / \psi K^{ \pm}$data, and the $p_{T}$ spectra of the two modes.

A final obstacle to the measurement is understanding the fraction of events in the $B^{ \pm} \rightarrow J / \psi K^{ \pm}$signal where the $J / \psi$ subsequently decays to two muons (as opposed to two electrons). This is non-trivial since the electrons are more prone to bremsstrahlung, shifting the reconstructed masses to lower energies. A log-likelihood fit is used to determine the $J / \psi \rightarrow \mu^{+} \mu^{-}$fraction in the measured $B^{ \pm} \rightarrow J / \psi K^{ \pm}$ signal. Additional cross-checks are then done to verify the fit result.

The remaining systematic uncertainties associated with this measurement are dis- 
cussed in Chapter 6.

\subsection{Candidate Selection}

\subsubsection{Track Quality Cuts}

All tracks used in this analysis were required to pass a set of basic track quality cuts before use. These cuts are:

- at least 25 axial and 25 Stereo COT hits

- at least $3 r-\phi$ hits in the silicon

- $p_{T}>400 \mathrm{MeV} / \mathrm{c}$

- $|\eta|<1.4$

\subsubsection{Trigger Confirmation}

During the event reconstruction described in Section 5.2.3 sets of three tracks within each event are used. Since the trigger efficiency is used in the final relative branching ratio calculation, it is important to verify that the tracks used in the analysis actually would have fired the trigger. There are many tracks generated by a $p \bar{p}$ collision in the Tevatron and "volunteer" events that triggered via other tracks in the event (those not used to reconstruct the decay of interest) should be excluded. The tracks generated by the SVT for fast decision-making in the Level-2 trigger are not the same as those finally made by the offline code. Therefore, performing trigger validation using the final offline tracks, while a reasonable approximation, is not technically accurate. The SVT-tracks used in Level-2 are recorded for later use, however, and can be used to verify the trigger. The offline candidate tracks are matched to those from the SVT based on their proximity in curvature and $\phi_{0}[47,46]$. The matched SVT-track is also required to have a $\chi^{2}<25$. After SVT-tracks have been matched to the offline candidate tracks, the trigger validation cuts are applied 

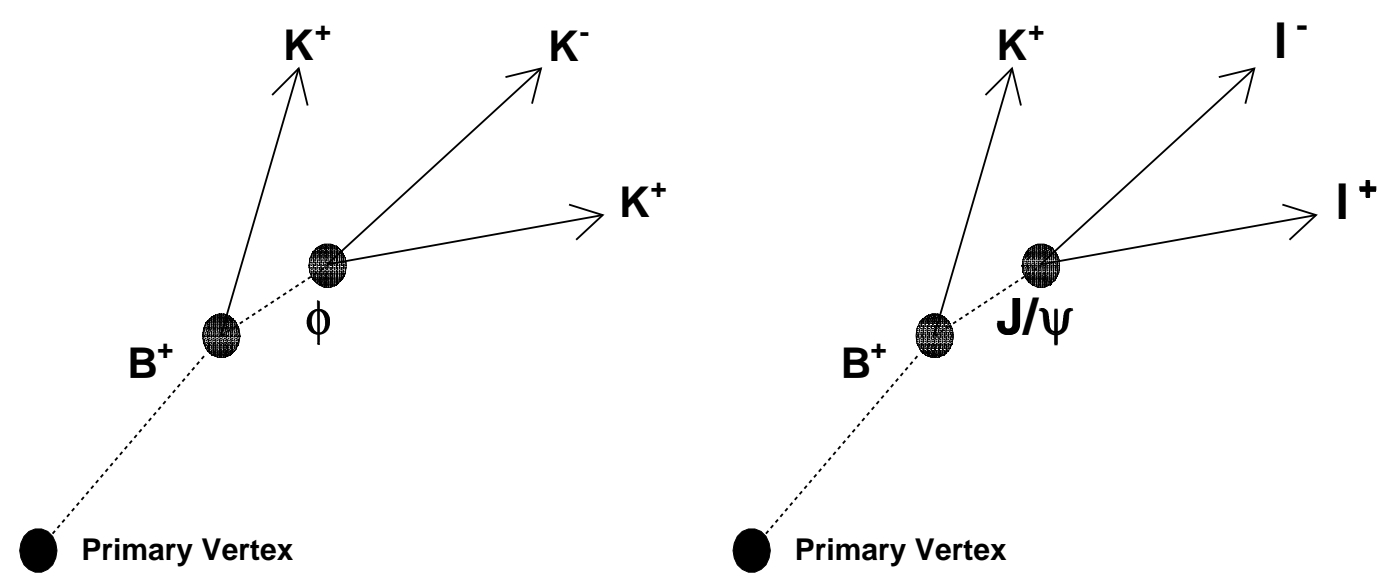

Figure 5.1: Decay topologies for the decays considered in this analysis. Left: $B^{ \pm} \rightarrow$ $\phi K^{ \pm}$with the subsequent decay $\phi \rightarrow K^{+} K^{-}$. Right: $B^{ \pm} \rightarrow J / \psi K^{ \pm}$with the subsequent decay $J / \psi \rightarrow l^{+} l^{-}$, where $l=(\mu, e)$.

to the associated SVT-track parameters. Of course, only two of the three candidate tracks need to satisfy the Two-Track Trigger cuts for this validation. The trigger bits were retained in the analysis ntuple as well as a flag indicating which trigger path would have fired based on the SVT track parameters associated with the actual candidate.

\subsubsection{Event Reconstruction}

The analysis presented here is based on a CharmMods executable corresponding to the version current at the time of the Spring 2003 conferences results, with all the bug fixes applied[48]. ${ }^{1}$ A parallel analysis based on the Universal Finder package[49] gives essentially the same results.

Fig. 5.1 shows the decay topologies reconstructed in this analysis. In the Tevatron,

\footnotetext{
${ }^{1}$ Technical Details: the version used is built with CharmMods/src/DFinder/TrackAssocModule.cc v1.4 and CharmMods/src/DFinder/TrackAssoc.cc v1.6 for retrieving calibrated dE/dx measurement as in [50], and on version v1.4 of svtsim/src/svtsim_pattern_finder.cc for matching an offline track to an SVT track, with a minor fix to allow matching for tracks that cross between SVX barrels. The executable is compiled against version 4.9.1hpt3 of the AC++ software.
} 
B hadrons are produced at the $p \bar{p}$ interaction point, also known as the primary vertex. B hadrons are relatively long-lived for unstable particles, having a mean lifetime on the order of $10^{-12} s$. At the typical momenta they are created with in the Tevatron they can travel as far as a few millimeters before decaying, with their decay products forming a discernible secondary vertex. Some of the decay products can subsequently decay again. These subsequent decays can form tertiary vertices if the intermediate particles are long lived, or they can appear to decay at a location indistinguishable from the secondary vertex (B hadron decay point) if they are short-lived. The intermediate decays of the $\phi$ and $J / \psi$ in this analysis are of the latter type (their average lifetimes are several orders of magnitude smaller than that of the B hadrons).

The final decay products pass through the detector and, provided they are charged, can be tracked. Those tracks can be used to determine the particle momenta, and be traced back to the original parent event. The transverse decay length, $L_{x y}$, of the parent particle can be found. If we apply a mass hypothesis on the tracked particles (e.g. assume they are kaons) we can calculate the relativistic energies for the daughter tracks, and use those values-along with the daughter track momenta-to reconstruct the momentum 4 -vector of the parent. This in turn allows us to calculate an invariant mass for the parent particle(s). Charge constraints are also imposed on the decay products, such that charge is conserved in each decay. This process is commonly referred to as "event reconstruction".

In this analysis, all three-prong vertices composed of tracks passing the previously described quality and trigger cuts were reconstructed. The vertex fitting was done using the CTVMFT module. Briefly described, the algorithm takes the original tracks consisting of five parameters, $\vec{\xi}=\left(c, \phi_{0}, \cot \theta, d_{0}, z_{0}\right)$, and redefines them as functions of three parameters, $\vec{\alpha}=\left(c, \phi_{0}, \cot \theta\right)$, and one common set of vertex coordinates, $(x, y, z)$. (Assuming all tracks are from the same vertex.) The vertex coordinates $(x, y, z)$ are then obtained by minimizing the $\chi^{2}$ of the fit[51].

There are many tracks reconstructed for each event in a $p \bar{p}$ collider. Aside from those made by actual B-hadron decays, there are also tracks from other sources: fragmentation particles made during the b-quark hadronization process, underlying events, and pile-up events. These tracks are responsible for a combinatoric back- 

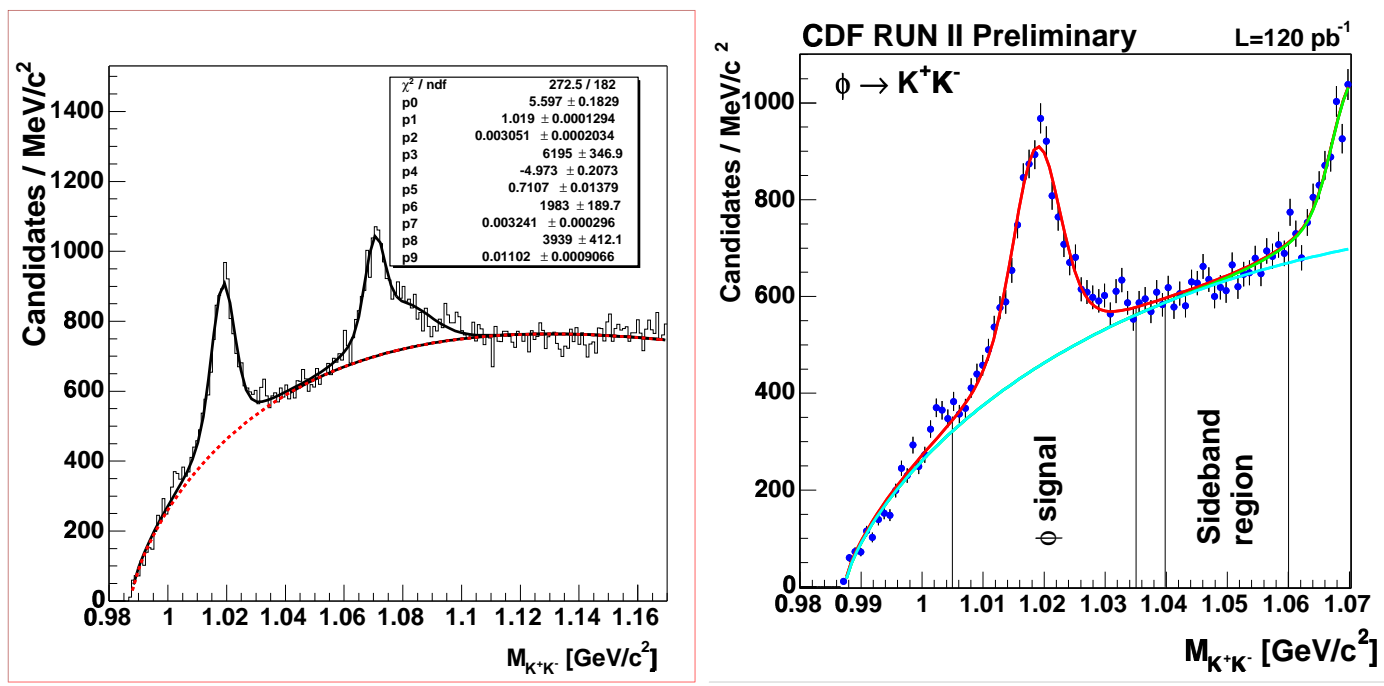

Figure 5.2: Left: $M_{K K}$ invariant mass for vertices in the B mass region Right: Zoomed in view of the left plot; red line: $\phi$ signal; cyan line: combinatorial background; green line: $K_{s}$ reflection.

ground present after signal reconstruction. To help distinguish the signal from these background events, further cuts are required. They are described in the next section.

\subsection{4 $B^{ \pm} \rightarrow \phi K^{ \pm}$Candidate Selection}

Given the tiny branching ratio for this mode (on the order of $10^{-6}$ ) at most a few tens of events are expected in the whole dataset. Thus for maximum efficiency the cuts to suppress the background were kept minimal.

Fig. 5.2 shows the invariant mass distribution for the two tracks postulated to come from the decay of the $\phi$, where the three track invariant mass (hypothesized $B^{ \pm}$mass) for the candidate event is between 4.8 and $6.0 \mathrm{GeV} / \mathrm{c}^{2}$. A clear peak from the $\phi \rightarrow K^{+} K^{-}$signal can be seen around $1.02 \mathrm{GeV} / \mathrm{c}^{2}$. Another peak can be seen around $1.07 \mathrm{GeV} / \mathrm{c}^{2}$. This satellite peak comes from $K_{s}^{0} \rightarrow \pi^{+} \pi^{-}$events with a mis-assigned kaon mass for the two pion tracks (the true mass of the $K_{s}^{0}$ is 498 $\left.\mathrm{MeV} / \mathrm{c}^{2}\right)$. A cut around the $\phi$ signal region $\left(1.015<M_{K K}<1.035 \mathrm{GeV} / \mathrm{c}^{2}\right)$ will be used to enhance the $B^{ \pm} \rightarrow \phi K^{ \pm}$signal. Also, data from the $\phi$ sideband region $\left(1.04<M_{K K}<1.06 \mathrm{GeV} / \mathrm{c}^{2}\right)$ will be used to model the combinatorial background 

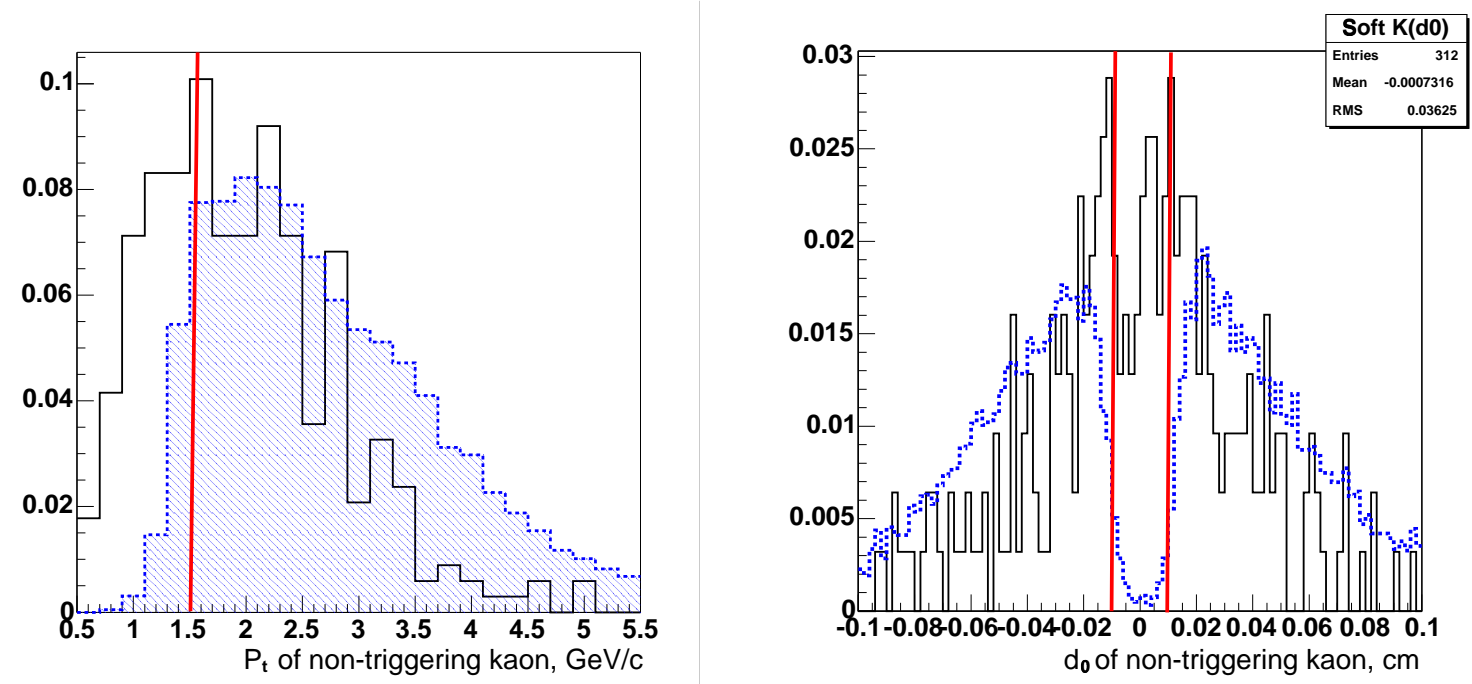

Figure 5.3: Left: Pt of the kaon not belonging to the trigger pair. Open histogram: data from $M_{\phi K}$ sidebands; Hatched histogram: $B^{ \pm} \rightarrow \phi K^{ \pm}$Monte Carlo. Right: Impact parameter for kaon not belonging to the trigger pair. Open histogram: data from $M_{\phi K}$ sidebands; Dashed histogram: $B^{ \pm} \rightarrow \phi K^{ \pm}$Monte Carlo

underneath the $\phi$ signal peak.

Further cuts to suppress the background were studied on a Monte Carlo sample of the $B^{ \pm} \rightarrow \phi K^{ \pm}$signal. The $I P(B)$ (B impact parameter), the non-trigger kaon $P t$ and the non-trigger kaon impact parameter distributions were compared to the corresponding data distributions obtained from the $M_{\phi K}$ sidebands defined as $80 \mathrm{MeV} / \mathrm{c}^{2} \leq\left|M_{\phi K}-5.28\right| \leq 160 \mathrm{MeV} / \mathrm{c}^{2}$ (corresponding to approximately a 4 sigma mass window 4 sigma away from the B mass). Examples of these comparisons are shown in Fig. 5.3.

The following cuts have been applied:

- $\Delta Z<5 \mathrm{~cm}$

- $\left|\phi_{\text {mass }}-1.02\right|<0.015 \mathrm{GeV}$

- $L_{x y}>0.02 \mathrm{~cm}$

- $I P(B)<0.01 \mathrm{~cm}$

- $d 0>0.01 \mathrm{~cm}$ for all three kaon tracks 
- $p_{T}>1.5 \mathrm{GeV} / \mathrm{c}$ for the non-triggering kaon

- Isolation $>0.5$

- $\chi_{x y}^{2}<20$

The Isolation variable is defined as the ratio between the sum of the candidate tracks' transverse momenta divided by the sum of the same quantity plus the transverse momenta of all tracks within a cone in eta and phi of radius 1 around the $B$ candidate's flight direction. The normalization mode $B_{u} \rightarrow J / \psi K$ is used to monitor the inefficiency introduced by this cut, which is at the $10 \%$ level. Since we are interested in the ratio of the two efficiencies the actual values are not really needed, but a correction factor based on the slightly different Pt spectra of the B candidates for the normalization mode and the signal mode must be evaluated. This is done in Section 6.3.

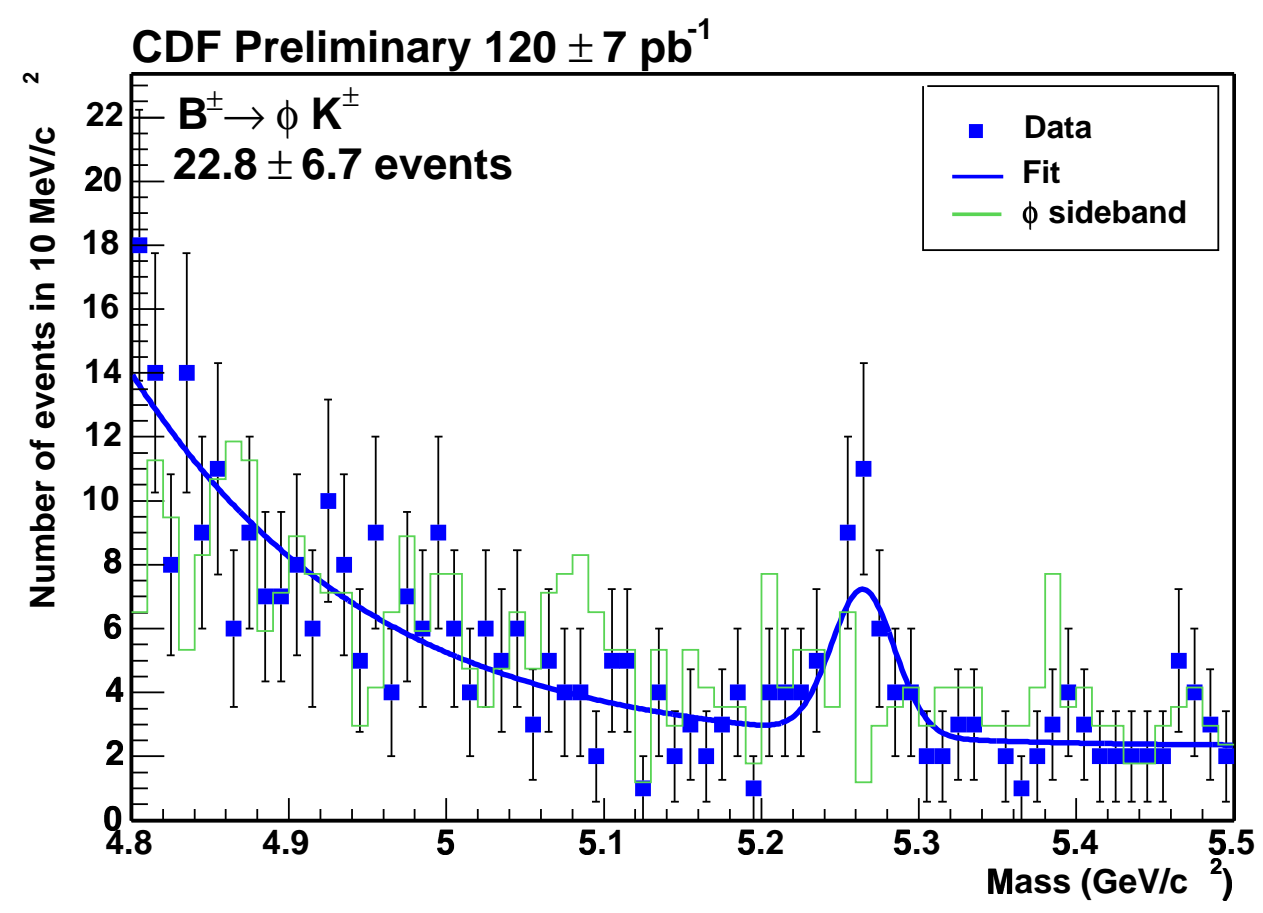

Figure 5.4: Mass plot of $B \rightarrow \phi K$ candidate events. 
The final B candidate invariant mass distribution is shown in Fig. 5.4. The fit was made using a Gaussian signal with a linear plus exponential background. The Gaussian width was constrained to $\sigma=23 \mathrm{MeV} / \mathrm{c}^{2}$ and yielded $22.8 \pm 6.7$ events. The present Monte Carlo predicts an $18 \mathrm{MeV} / \mathrm{c}^{2}$ width for the signal but this value was increased by an additional $15 \%$ based on data/Monte Carlo comparisons for other modes seen at CDF, specifically $D_{s} \rightarrow \phi \pi$ and $B^{ \pm} \rightarrow J / \psi K^{ \pm}$as shown in Section 6.1.

The $\phi$ sideband data, normalized to the upper B mass sideband, are also superimposed on this plot. These data support the exponentially decreasing background for the candidate events. The excesses seen on the lower B sideband are believed to be from other $B \rightarrow \phi X$ partially reconstructed decays.

\subsection{The $B^{ \pm} \rightarrow J / \psi K^{ \pm}$Normalization Mode}

The same cuts that were used to find the $B^{ \pm} \rightarrow \phi K^{ \pm}$candidates were used to find the normalization mode, $B^{ \pm} \rightarrow J / \psi K^{ \pm}$, except that in place of the $\phi$ mass the mass of the $J / \psi$ was used $\left(\left|J / \psi_{\text {mass }}-3.094\right|<0.030 \mathrm{GeV}\right)$ and the tracks from the $J / \psi$ were postulated to be muons. The reason for the difference in the $J / \psi$ mass used from the Particle Data Group[54] value of $3.097 \mathrm{GeV} / \mathrm{c}^{2}$ is that the tracks were Kalman refit as kaons, not muons, so a correction to the mass must be applied. This is valid since the yields are being measured, not the masses.

The results of this search are shown in Fig. 5.5, with $406 \pm 26$ events found. To be better able to make a direct comparison with the $\phi$ decay reconstruction in Section 5.2.4 no calorimeter or muon chamber information was used in reconstructing the $J / \psi$.

The fit was made using a Gaussian superimposed on a background consisting of a linear function plus an error function.

$$
\left.N_{B} \cdot G\left(M_{B}, \sigma_{B}\right)+\operatorname{Poly1}(\text { constant }, \text { slope })+\text { plateau } \cdot \text { Erf(edge }\right)
$$

The functional form of this background, with a "turn on" at about one pion mass below the peak, was verified by looking at a generic fully simulated Monte Carlo sample containing the decays $B_{0}, B_{s}, B^{ \pm}, \Lambda_{b} \rightarrow J / \psi+X$ followed by $J / \psi \rightarrow \mu \mu$, 


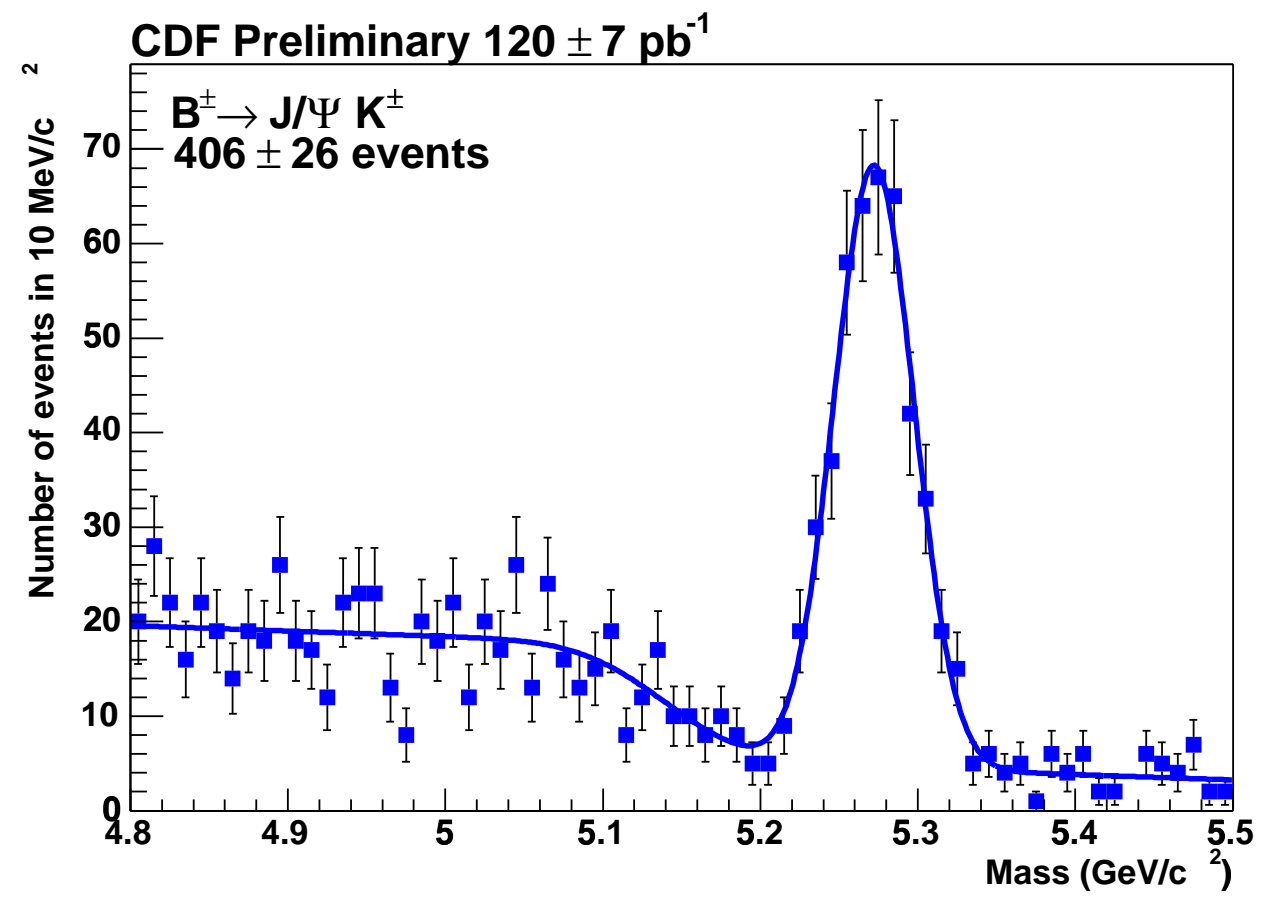

Figure 5.5: Mass plot of $B \rightarrow J / \psi K$ events. 
obtained using Bgenerator. These results can be seen in Fig. 5.6. In this case, the fit does not include the linear term for the combinatorial background, which is not present in the simulation.

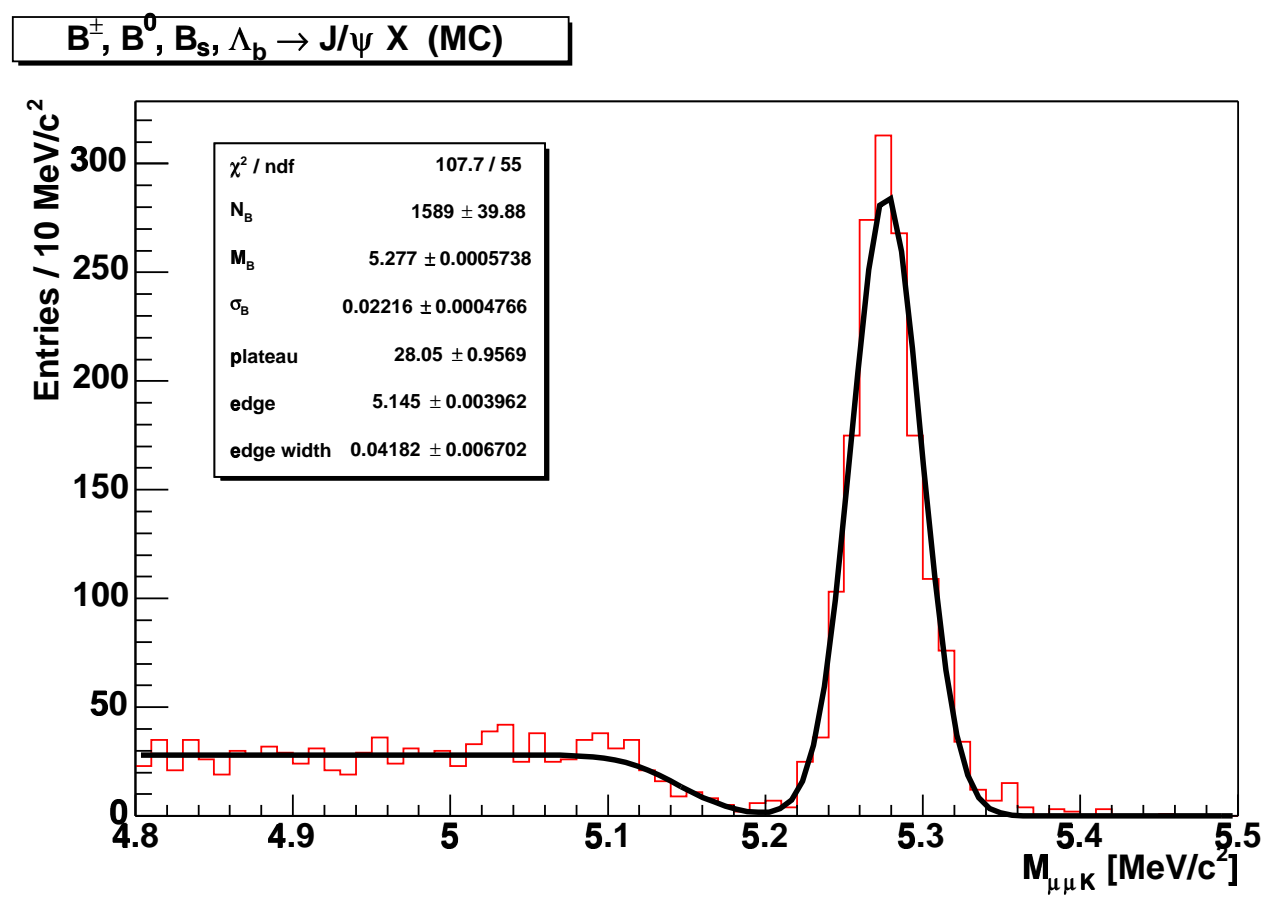

Figure 5.6: Mass plot of $B \rightarrow J / \psi K$ from realistic Monte Carlo containing the decays $B_{0}, B_{s}, B^{ \pm}, \Lambda_{b} \rightarrow J / \psi+X$.

\subsection{Relative Efficiencies from Monte Carlo Simu- lation}

The relative acceptances and trigger efficiencies of the three decay configurations under study were calculated using Monte Carlo data. The Monte Carlo events were generated as described in Section 4.2 In Fig. 5.7 the $p_{T}$ spectrum of Monte Carlo events is compared with the $p_{T}$ spectrum of data in the $B^{ \pm} \rightarrow J / \psi K^{ \pm}$sample after the same trigger, reconstruction and analysis cuts. The cuts were the same as described in Section 5.2.4 with the exception of the isolation cut which can not be simulated, 


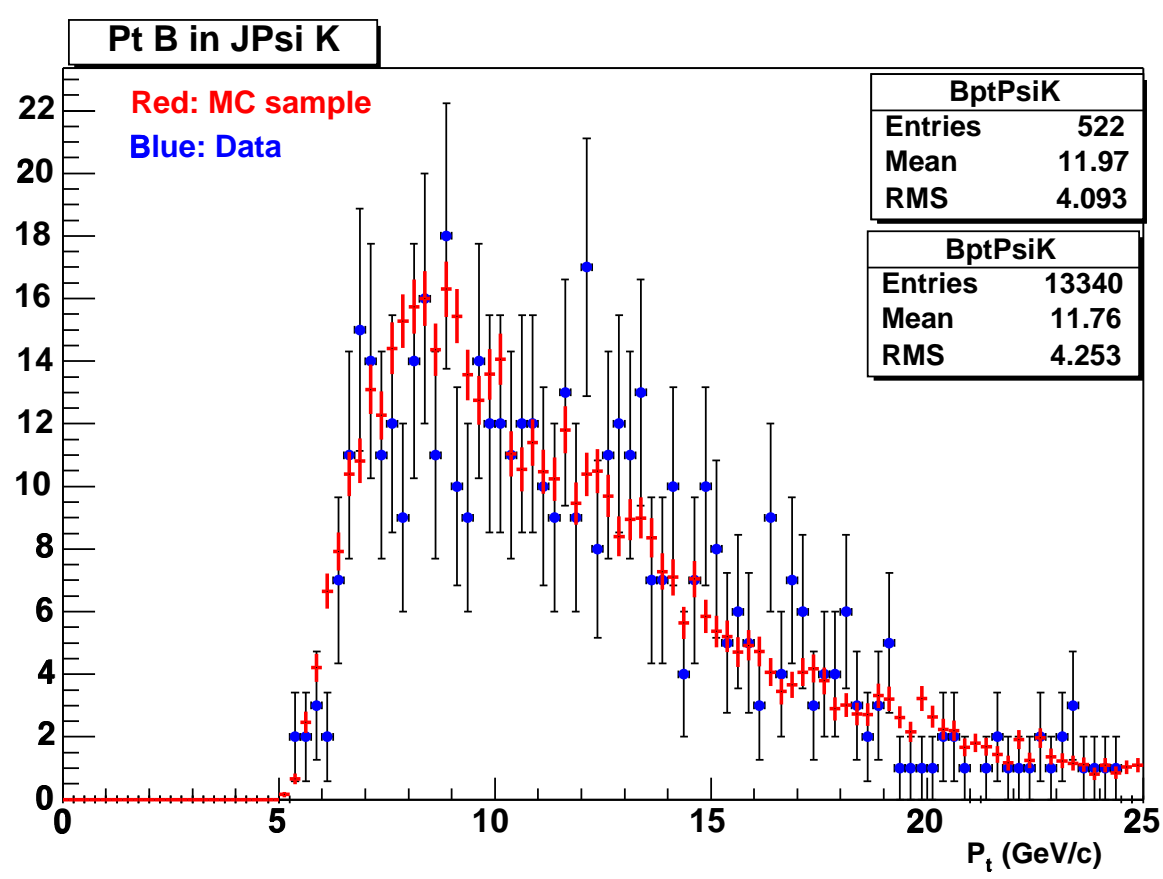

Figure 5.7: The $B^{ \pm} \rightarrow J / \psi K^{ \pm}$Pt spectrum in Monte Carlo and data.

and was not used. Both electron and muon events are included in these spectra. Good agreement is seen between the Monte Carlo prediction for the B transverse momentum spectra and data.

Tables 5.1, 5.2, and 5.3 show the trigger, reconstruction and total efficiency after the analysis cuts for the $B^{ \pm} \rightarrow \phi K^{ \pm}$and $B^{ \pm} \rightarrow J / \psi K^{ \pm}$signals. In the $B^{ \pm} \rightarrow$ $\phi K^{ \pm}$simulation the reconstruction efficiency (basically the request of three silicon outside-in tracks in the vertex) varies from $75 \%$ in the earlier data (with a worse SVX coverage) to $80 \%$ in the latter part of the data taking period. The analogous number for the normalization mode is around $60 \%$. The average analysis efficiency is around $80 \%$ for the $B^{ \pm} \rightarrow \phi K^{ \pm}$signal and $60 \%$ for the $B^{ \pm} \rightarrow J / \psi K^{ \pm}$signal. The harder $P t$ spectra of the particles from the $B^{ \pm} \rightarrow \phi K^{ \pm}$decays is the main reason for the higher efficiency in that mode.

Fig. 5.8 shows the ratio of the total efficiencies for $K K K$ and $\mu \mu K$ for the eight representative run numbers used in the simulation. Since no significant variation of 


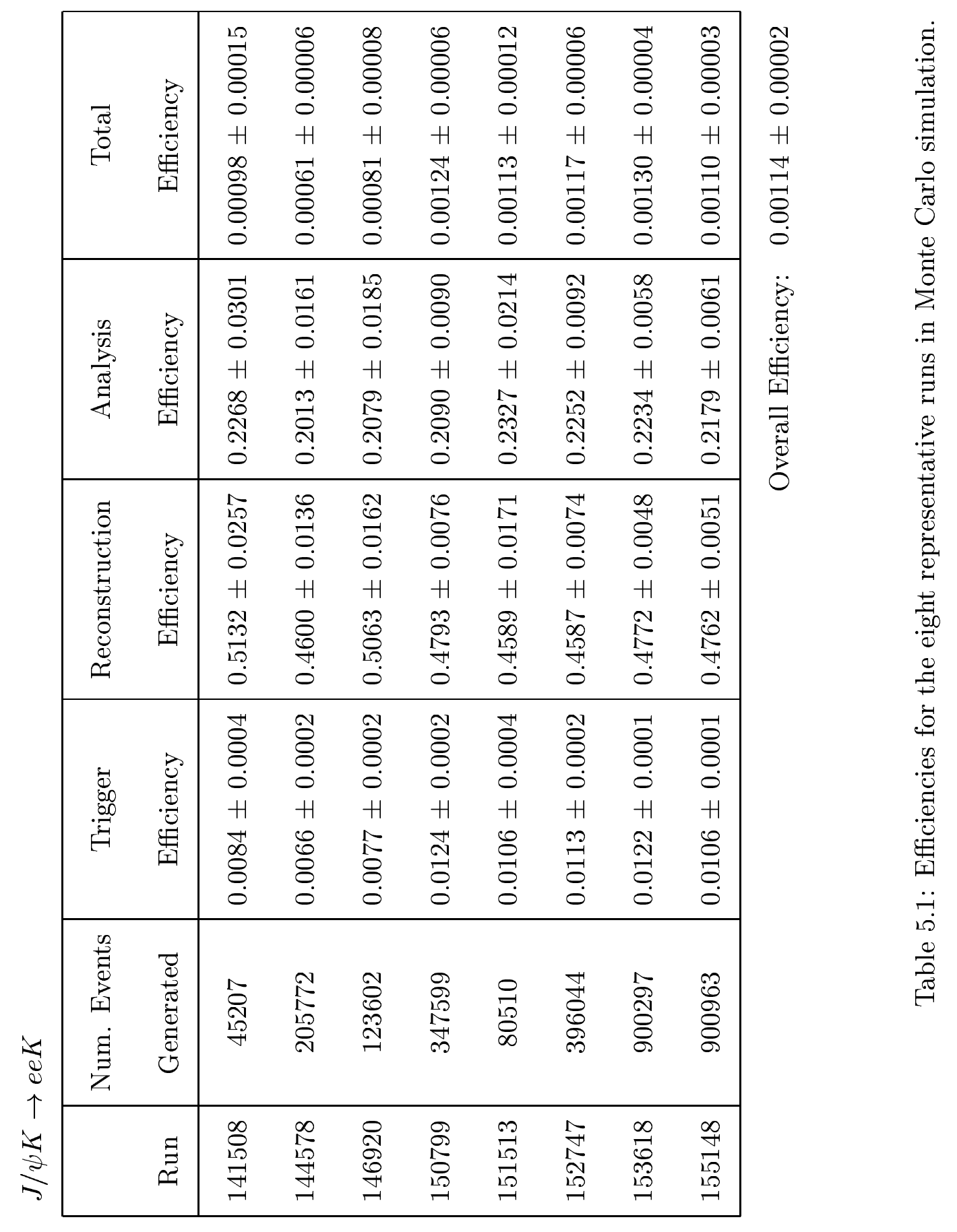




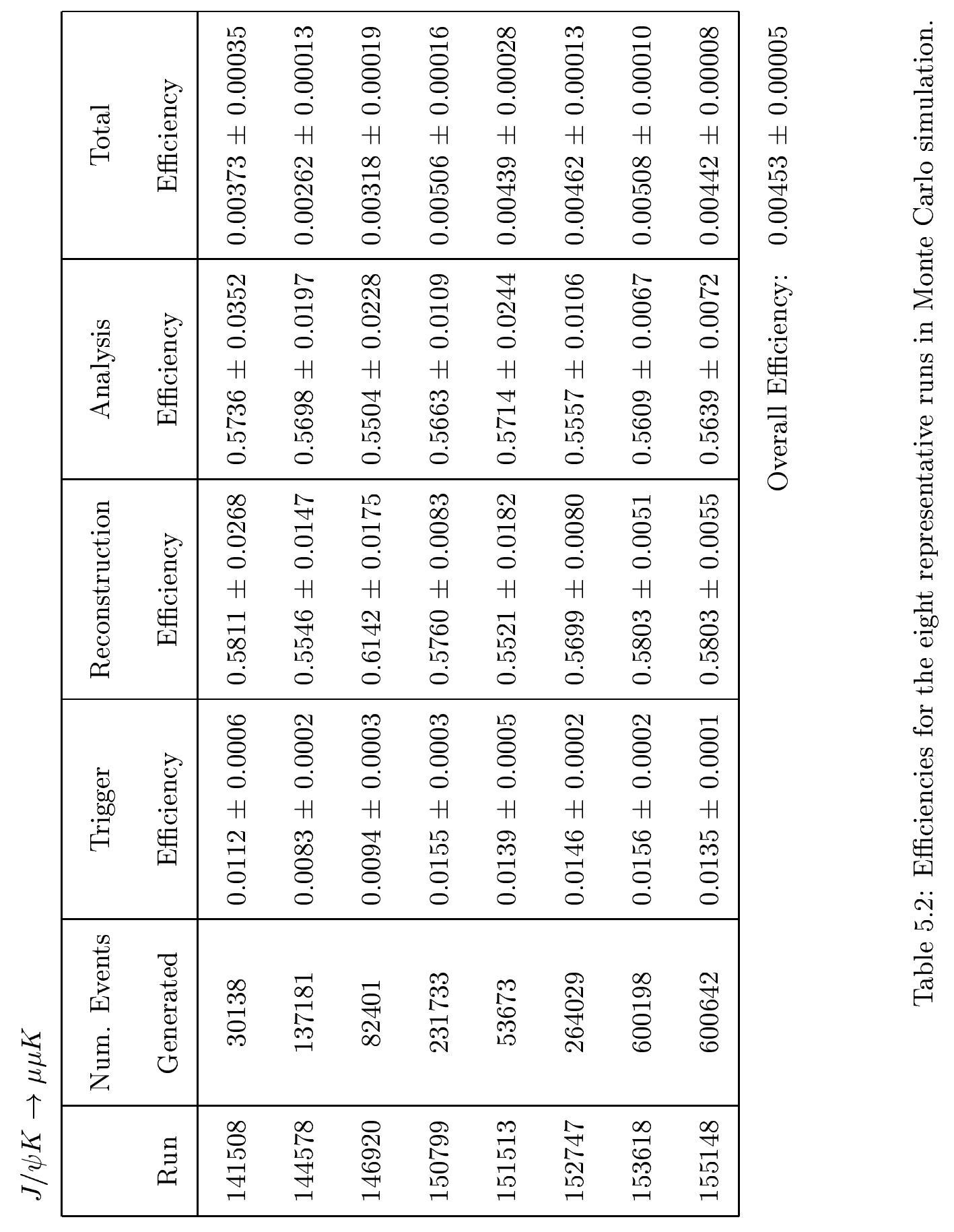




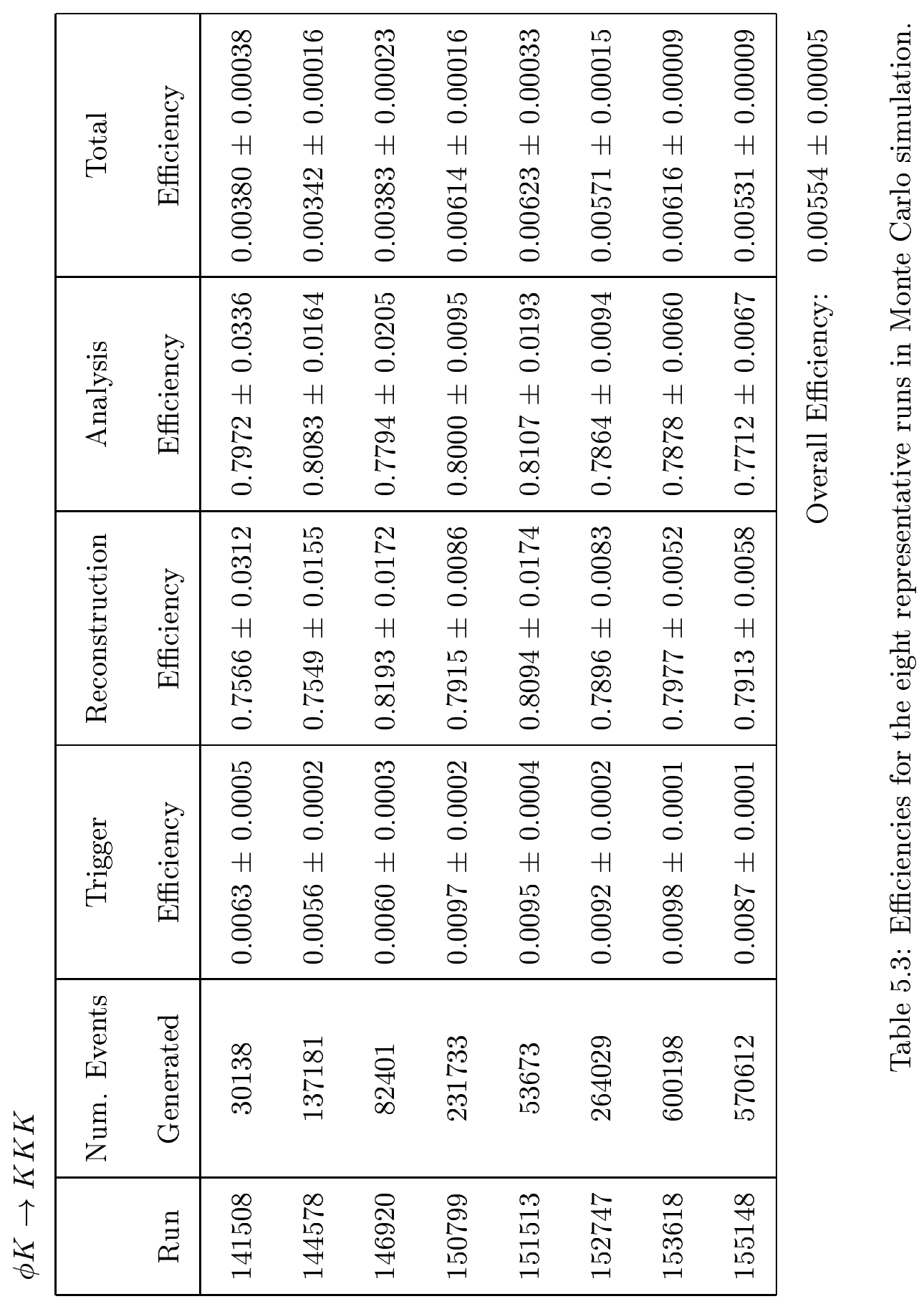


the efficiency ratio with run number is observed, the overall efficiencies (weighted averages over the eight simulated runs) are used in the final relative branching ratio calculation.

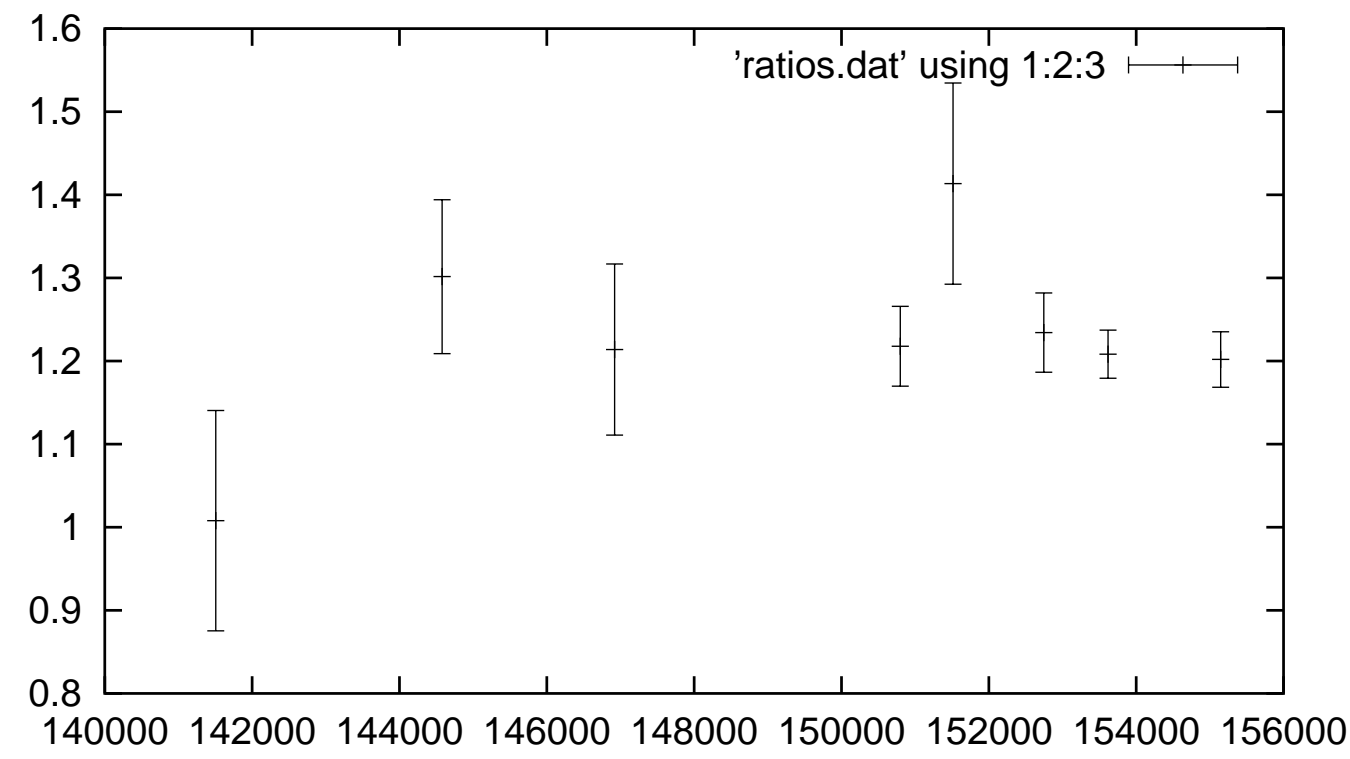

Figure 5.8: Ratio of $\epsilon_{\phi K} / \epsilon_{\psi K}$ for the eight run numbers used in simulation.

The efficiency of the $\chi_{x y}^{2}$ cut is found to not be fully reproduced by the Monte Carlo. In addition, there might be a difference in the $\chi_{x y}^{2}$ efficiency across the different channels due to slightly different vertex topology. The systematic error to be associated with this effect is evaluated in Section 6.4.

\subsection{Finding the Fraction of $J / \psi \rightarrow \mu^{+} \mu^{-}$Events in the $B^{ \pm} \rightarrow J / \psi K^{ \pm}$Signal}

A major concern in this analysis is finding the fraction of events in the $B^{ \pm} \rightarrow$ $J / \psi K^{ \pm}$mass peak which come from $J / \psi \rightarrow \mu^{+} \mu^{-}$(vs. $J / \psi \rightarrow e^{+} e^{-}$). This is nontrivial since the electrons bremsstrahlung in the detector material and are shifted toward lower momentum therefore causing the parent $J / \psi$ to be reconstructed with 
much lower efficiency than those that decay to muons.

The $J / \psi \rightarrow \mu^{+} \mu^{-}$fraction was determined by simultaneously applying a fit on the $\mathrm{B}$ mass, $J / \psi$ mass, and the $d E / d x$ values from the COT for each of the two $J / \psi$ tracks. In addition to this 4-dimensional fit, three other (partially correlated) methods were used to determine the muon fraction as cross-checks:

- A Monte Carlo prediction was computed using the sample described previously.

- Muon chamber information was applied, along with efficiency estimates from the Monte Carlo for track-muon stub matching.

- A simpler, 2-dimensional fit using only the $d E / d x$ values from the COT to distinguish muons from electrons.

Each of these methods will be more fully described in the following subsections, and the results summarized afterward.

\subsubsection{Fraction of $J / \psi \rightarrow \mu^{+} \mu^{-}$From the 4-Dimensional Fit}

An extended, multi-variable, log likelihood fit was applied which simultaneously fit on the candidate's B and $J / \psi$ invariant masses and the $\mathrm{dE} / \mathrm{dx}$ values ${ }^{2}$ for the two tracks from the $J / \psi$.

In an extended log-likelihood fit the normalized probability distribution functions (PDFs), $\rho_{i}(\vec{x} ; \vec{a})$ where $\vec{x}$ are the measured variables and $\vec{a}$ are the parameters of the distribution, are extended by a factor $n_{i}$, i.e.

$$
P_{i}\left(\vec{x} ; \vec{a}, n_{i}\right)=n_{i} \cdot \rho_{i}(\vec{x} ; \vec{a})
$$

This is most appropriate when measuring yields and the exact number of each category of event is unknown[53]. In the simplest case there will be two categories of events: signal and background. Notice that the extension factor is now a parameter of the new "PDF-like" term. The extended likelihood for $\mathrm{N}$ events now becomes

\footnotetext{
${ }^{2} \mathrm{All} \mathrm{dE} / \mathrm{dx}$ values were calculated under a pion hypothesis.
} 


$$
\mathcal{L}_{N}=\exp \left(-\sum_{i} n_{i}\right) \prod_{j=1}^{N}\left(\sum_{i} P_{i}\left(\overrightarrow{x_{j}} ; \vec{a}, n_{i}\right)\right),
$$

the maximization of which leads to $\sum n_{i}=N$.

The fit contains five parts: signal events from $J / \psi \rightarrow \mu^{+} \mu^{-}$, signal events from $J / \psi \rightarrow e^{+} e^{-}$, background events containing $J / \psi \rightarrow \mu^{+} \mu^{-}$not from $B^{ \pm} \rightarrow J / \psi K^{ \pm}$, background events containing $J / \psi \rightarrow e^{+} e^{-}$not from $B^{ \pm} \rightarrow J / \psi K^{ \pm}$, and all other combinatorial background events. The PDFs used for each of these cases are described in detail later in this section, but first a brief discussion of the data samples used to determine the (fixed) shape parameters for those PDFs is in order.

The muon and electron PDFs were found using $J / \psi \rightarrow \mu \mu$ and $J / \psi \rightarrow$ ee samples derived from datasets that were generated by triggers specifically designed to select these types of events ${ }^{3}$. Both data samples were selected using the CharmMods package. Tracks were required to have at least 20 axial and 20 stereo COT hits and $3 \mathrm{Si}$ $R$ - $\phi$ hits, $P_{T}>1.5 \mathrm{GeV} / c$ and $|\eta|<1.5$. The refitting of these tracks was performed as described in Section 4.3.2.

The $d E / d x$ pull distributions are defined as $\left(\left.\frac{d E}{d x}\right|_{\text {meas }}-\left.\frac{d E}{d x}\right|_{\pi}\right) / \sigma$, where $\left.\frac{d E}{d x}\right|_{\pi}$ is the value expected for the particle under a pion hypothesis. Log-normal functions were used to describe the 1-dimensional $d E / d x$ pull PDFs for the different particle types. The parameters used for each different particle type and track charge are shown in Table 5.4. These parameters have been measured using independent high-statistics samples and were not allowed to vary during the fit to determine muon fraction. The PDF parameters for pions and kaons were taken from [50] where $D^{* \pm}$ decays are used to obtain pure samples of those particles, while proton and antiproton parameters were determined using a $\Lambda^{0}$ sample ([55]). Clean samples of $J / \psi \rightarrow \mu \mu$ and $J / \psi \rightarrow$ ee were obtained using the cuts described in Table 5.5. A $3 \sigma$ window around the $J / \psi$ reconstructed mass was selected and the background from both sideband regions was subtracted.

In this analysis $P_{x}^{c}$ will represent the $d E / d x$ pull PDF for a particle of type $x$ with

\footnotetext{
${ }^{3}$ The $J / \psi \rightarrow \mu \mu$ events were derived from the jbotoh dataset described in [56], and the $J / \psi \rightarrow$ ee events were derived from the $\mathrm{j}$ bot $7 \mathrm{~h}$ dataset described in [57].
} 


\begin{tabular}{|cc|c|c|c|c|}
\hline \hline \multirow{2}{*}{ Track } & Shape & Ln Scale & Pull & Pull \\
& Charge & Parameter, $\sigma$ & Parameter, $\mu$ & Mean & Variance \\
\hline \hline pion & + & 2.2982 & 0.1024 & 0.0083 & 1.028 \\
& - & 2.3039 & 0.1021 & 0.0655 & 1.030 \\
\hline kaon & + & 2.1624 & 0.1134 & -1.252 & 0.995 \\
\hline electron & + & 2.4870 & 0.0960 & 2.080 & 1.160 \\
& - & 2.5060 & 0.0949 & 2.310 & 1.170 \\
\hline muon & + & 2.3170 & 0.1040 & 0.200 & 1.064 \\
\hline \hline \multirow{2}{*}{+} & 2.3140 & 0.1051 & 0.171 & 1.072 \\
\hline
\end{tabular}

Log-normal function: $F(x ; \mu, \sigma)=\frac{1}{\sqrt{2 \pi \sigma}} \cdot \frac{1}{x+10} \cdot e^{-\frac{1}{2}\left(\frac{\ln (x+10)-\mu}{\sigma}\right)^{2}}$

Table 5.4: Parameters from the fit of the $d E / d x$ pull distributions using the lognormal function written above (second and third column). The fourth and fifth column list the mean and variance of the pulls. 


\begin{tabular}{|l|l|}
\hline$J / \psi \rightarrow \mu^{+} \mu^{-}$ & $J / \psi \rightarrow e^{+} e^{-}$ \\
\hline$Q_{1} \cdot Q_{2}<0$ & $Q_{1} \cdot Q_{2}<0$ \\
$P_{1,2}^{T}>1.5 \mathrm{GeV} / c$ & $P_{1,2}^{T}>2 . \mathrm{GeV} / c$ \\
$\left|\eta_{1,2}\right|<1$ & $\left|\eta_{1,2}\right|<1$ \\
$\left|I P_{J / \psi}\right|<0.01 \mathrm{~cm}$ & $\left|I P_{J / \psi}\right|<0.01 \mathrm{~cm}$ \\
$\chi_{x y}^{2}<10$ & $\chi_{x y}^{2}<10$ \\
$P_{J / \psi}^{T}>4 \mathrm{GeV} / c$ & $E /\left.p\right|_{1,2}<1.5$ \\
& $E_{\mathrm{had}} /\left.E_{\mathrm{em}}\right|_{1,2}<0.05$ \\
& $|\Delta \cot (\vartheta)|>0.02$ \\
\hline \hline
\end{tabular}

Table 5.5: Cuts used in the $\mathrm{dE} / \mathrm{dx}$ analysis. $\Delta \cot (\vartheta)$ is the difference between the polar angle cotangent of the two tracks and $\Delta x y$ is the separation between the two track helices in the transverse plane. 
charge $c$. As an example the $d E / d x$ pull PDF for positron tracks, $P_{e}^{+}$, is shown in Fig. 5.9. The $d E / d x$ pull PDFs for all particles are shown in Fig. 5.10 on a log scale.

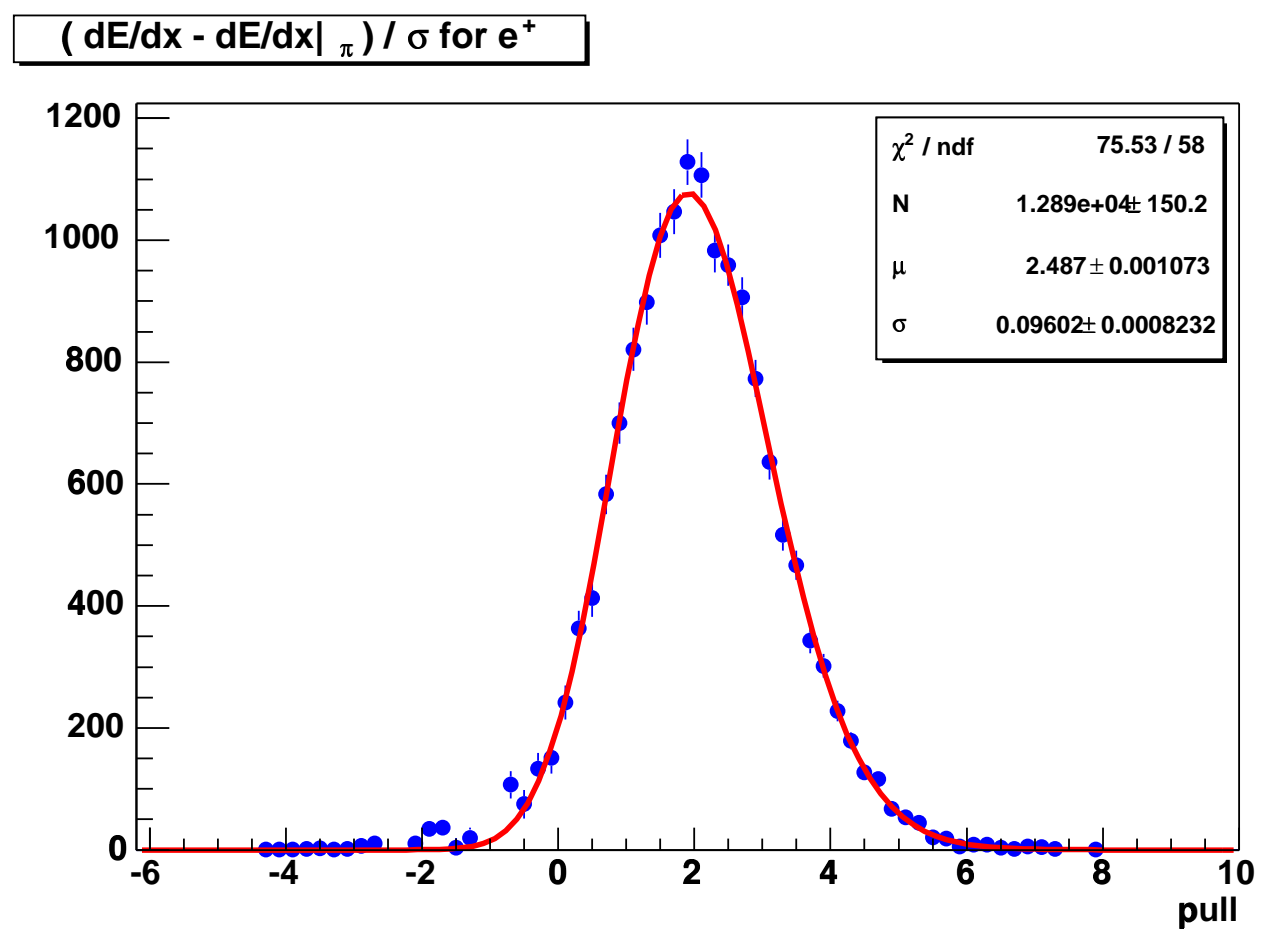

Figure 5.9: The $d E / d x$ pull PDF for positrons.

The muonic signal events, $B^{ \pm} \rightarrow J / \psi K^{ \pm}(J / \psi \rightarrow \mu \mu)$, were fit with the PDF $S_{\mu \mu}=G\left(M_{B} ; \mu_{B}, \sigma_{B}\right) \cdot G_{\Sigma 2}\left(M_{J / \psi} ; \mu_{J / \psi}\right) \cdot P_{\mu}^{+}($pos. track dE $/ \mathrm{dx}) \cdot \mathrm{P}_{\mu}^{-}($neg. track $\mathrm{dE} / \mathrm{dx})$ where $\mathrm{G}$ is a Gaussian function, and $G_{\Sigma 2}$ is the sum of two Gaussians.

$G_{\Sigma 2}=0.7033 \cdot G_{1}\left(M_{J / \psi} ; \mu_{J / \psi}, \sigma=0.0128\right)+0.2977 \cdot G_{2}\left(M_{J / \psi} ; \mu_{J / \psi}-0.004, \sigma=0.2\right)$ $M_{B}$ and $M_{J / \psi}$ are the invariant masses of the candidate's B meson and $J / \psi$, respectively. The fit parameters $\mu_{B}, \sigma_{B}$, and $\mu_{\mathrm{J} / \psi}$ represent the mean B mass, B mass width, and mean $J / \psi$ mass, respectively. This PDF is extended with the parameter $N_{\mu \mu K}$, the number of muonic signal events.

The PDF for $B^{ \pm} \rightarrow J / \psi K^{ \pm}$signal events decaying to electrons is

$$
S_{e e}=G_{\Sigma 6}^{2}\left(M_{B}, M_{J / \psi} ; \mu_{J / \psi}\right) \cdot P_{e}^{+} \cdot P_{e}^{-}
$$



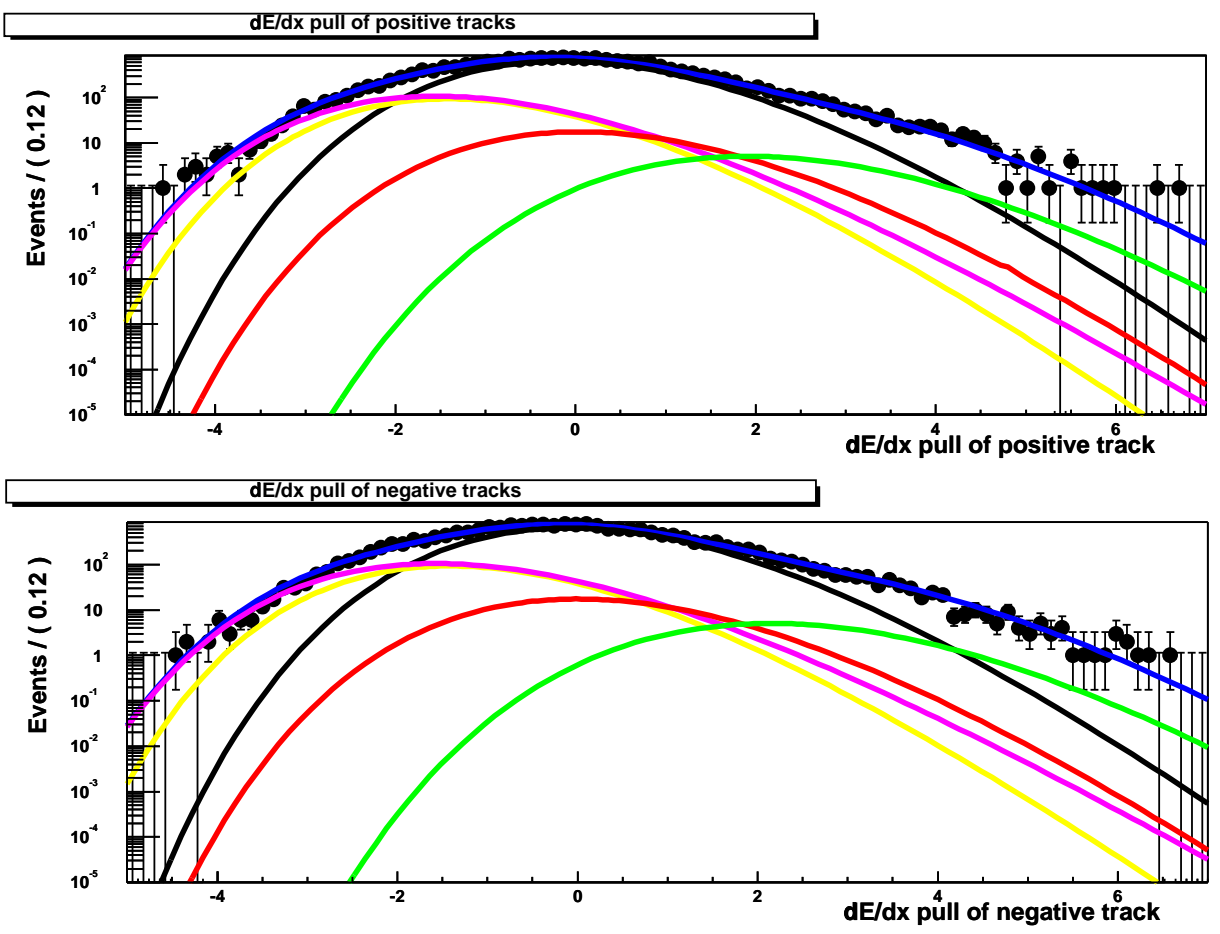

Figure 5.10: The dE/dx pull PDFs for all particles calculated under a pion hypothesis. Yellow: protons, Magenta: kaons, Black: pions, Red: muons, Green: electrons. The blue curve is the sum over all particles. 
where $G_{\Sigma 6}^{2}$ is a sum of six 2-dimensional Gaussians. This complicated functional form is necessary to model the bremsstrahlung tail of the electrons on the two dimensional mass plot, which can be seen in Fig. 5.11. There is still only one free parameter in this function, the mean mass of the $J / \psi$. The $S_{e e} \mathrm{PDF}$ is extended with the parameter $N_{e e K}$, the number of electronic signal events.

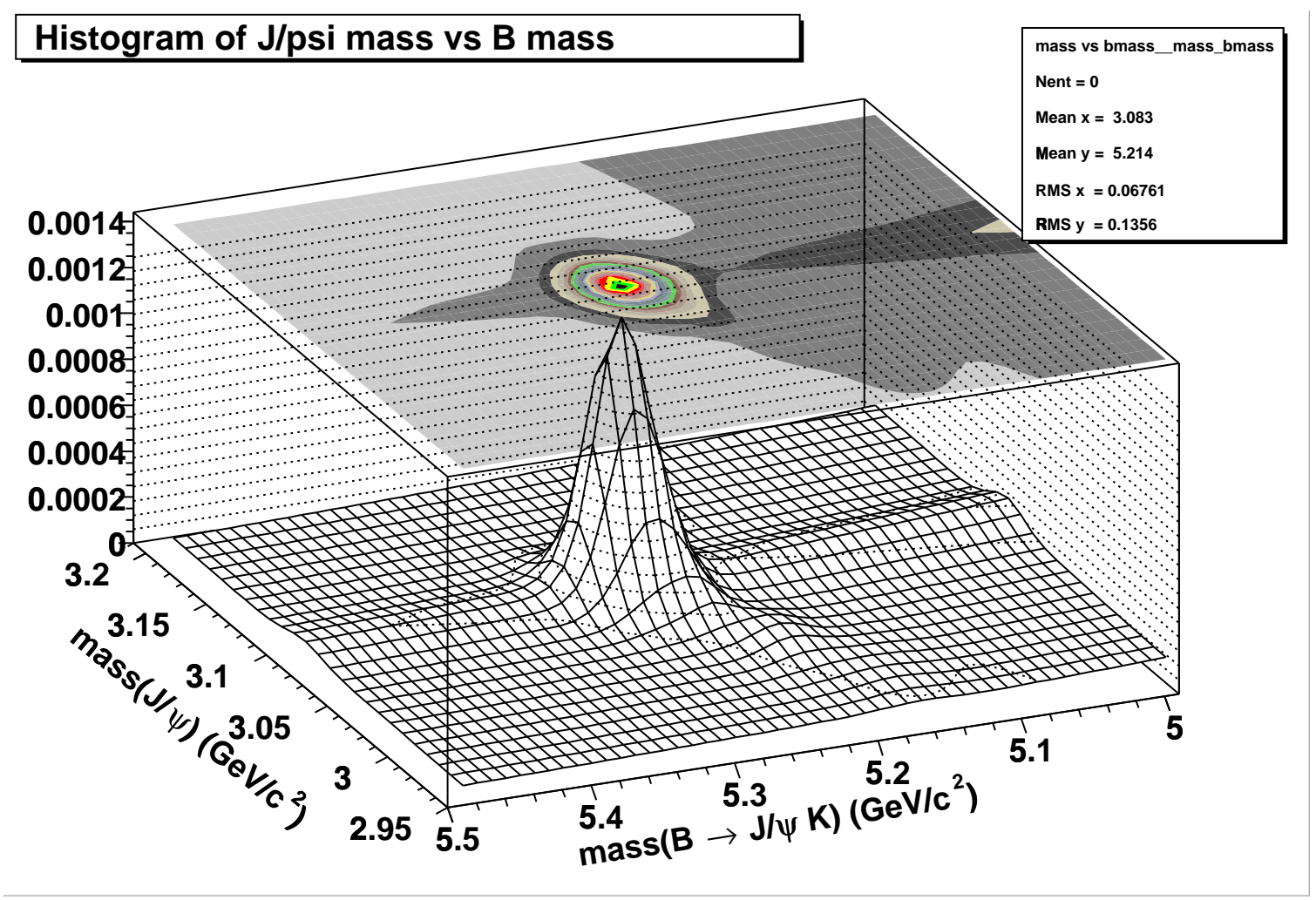

Figure 5.11: Histogram of $B_{\text {mass }}$ vs. $\mathrm{J} / \psi_{\text {mass }}$. Note the bremsstrahlung tail in the direction of decreasing $\mathrm{B}$ and $J / \psi$ masses, at a $45^{\circ}$ angle to both axes.

The PDF for background events containing $J / \psi \rightarrow \mu \mu$, where the $J / \psi$ is not from $B^{ \pm} \rightarrow J / \psi K^{ \pm}$, is

$$
\begin{aligned}
B_{\mu \mu}= & {\left[f_{\text {phys bkg }}\left(1-\frac{1}{1+\operatorname{Exp}\left(\frac{5.143-M_{B}}{0.034}\right)}\right)+\left(1-f_{\text {phys bkg }}\right) \operatorname{Exp}\left(M_{B} ; \tau\right)\right] \times } \\
& G_{\Sigma 2}\left(M_{J / \psi} ; \mu_{J / \psi}\right) \cdot P_{\mu}^{+} \cdot P_{\mu}^{-}
\end{aligned}
$$


Where $\tau$ is the coefficient of the exponential parameter. The term in parenthesis

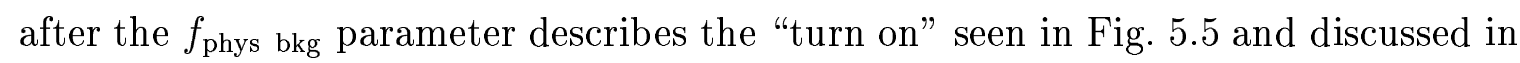
Section 5.3. This PDF is extended with the parameter $N_{u u K} \cdot R_{B / S}$, where $R_{B / S}$ is the ratio of background to signal events for $B^{ \pm} \rightarrow J / \psi K^{ \pm}$, i.e.

$$
R_{B / S}=\frac{\#(B \rightarrow J / \psi X)_{X \neq K}}{\#(B \rightarrow J / \psi K)}
$$

The PDF for background events containing $J / \psi \rightarrow e e$ where the $J / \psi$ is not from $B^{ \pm} \rightarrow J / \psi K^{ \pm}$is

$$
\begin{aligned}
B_{e e}= & {\left[f_{\text {phys bkg }}\left(1-\frac{1}{1+\operatorname{Exp}\left(\frac{5.143-M_{B}}{0.034}\right)}\right)+\left(1-f_{\text {phys bkg }}\right) \operatorname{Exp}\left(M_{B} ; \tau\right)\right] \times } \\
& {\left[0.1675 \cdot G\left(M_{J / \psi} ; \mu_{J / \psi}\right)+(1-0.1675) \cdot G_{b i f}\left(M_{J / \psi} ;\left(\mu_{J / \psi}-0.001\right)\right)\right] \times } \\
& \cdot P_{e}^{+} \cdot P_{e}^{-}
\end{aligned}
$$

where the $J / \psi$ mass PDF is the sum of a Gaussian and a bifurcated Gaussian ${ }^{4}$. The Gaussian has $\sigma=0.0126 \mathrm{GeV}$ and the bifurcated Gaussian has $\sigma_{L}=0.0612 \mathrm{GeV}$ and $\sigma_{R}=0.0170 \mathrm{GeV}$. This PDF is extended with the parameter $N_{e e K} \cdot R_{B / S}$.

All other combinatorial background events are modeled by

$$
B_{\text {other }}=\operatorname{Exp}\left(M_{B} ; \tau\right) \cdot \operatorname{Poly} 1\left(M_{J / \psi} ; p_{1}\right) \cdot P_{\text {other }}^{+} \cdot P_{\text {other }}^{-}
$$

where Poly1 is a first order polynomial and

$$
P_{\text {other }}^{ \pm}=f_{e} \cdot P_{e}^{ \pm}+f_{K} \cdot P_{K}^{ \pm}+f_{p} \cdot P_{p}^{ \pm}+\left(1-f_{e}-f_{k}-f_{p}\right) \cdot P_{\pi}^{ \pm}
$$

where $f_{e}, f_{K}$ and $f_{p}$ are the fractions of electrons, kaons and protons respectively in the combinatorial background. Since the fit in the sidebands doesn't have the separation to distinguish kaons from protons one of the values had to be set constant

\footnotetext{
${ }^{4} \mathrm{~A}$ bifurcated Gaussian is a Gaussian that has different widths for the left and right part of the curve, designated here as $\sigma_{L}$ and $\sigma_{R}$, respectively.
} 
for the fit to be stable, i.e $f_{p}=0.126$ and $f_{K}$ is a free parameter in the $\mathrm{fit}^{5}$. The general background PDF is extended with $N_{b k g}$, the number of background events.

The results of the fit are shown in Table 5.6. The projections of the fit onto the four different dimensions are shown in Figs. 5.12 to 5.14. Both the positive and negative charge $d E / d x$ pull projections are in very good agreement with the fitted results, as is the $J / \psi$ mass projection. The fit does not reproduce extremely well the $\mathrm{B}$ mass projection. Fig. 5.15 is the same projection made in the signal region (e.g. after applying a cut on the $J / \psi$ mass). It shows that in the region of interest the fit does reproduce the data well, and actually matches the total number of $B^{ \pm} \rightarrow J / \psi K^{ \pm}$ candidates to within a few percent.

From $S_{e e}$, the number of electrons in the signal region is found to be $0.6050 \cdot N_{e e K}$. Along with the fit results this gives a fraction of signal events that are from muons of

$$
f_{\mu \mu}^{f i t}=\frac{N_{\mu \mu}}{N_{e e} \times 0.605+N_{\mu \mu}}=0.839 \pm 0.016(\text { stat. })
$$

\subsubsection{Fraction of $J / \psi \rightarrow \mu^{+} \mu^{-}$From the 2-Dimensional Fit}

The muon and electron PDFs from the previous section were used again to perform a simplified fit where only $d E / d x$ information was used. Only events in the signal $B^{ \pm} \rightarrow J / \psi K^{ \pm}$window were used. The $d E / d x$ response for the combinatorial background from pions, kaons, and protons is modeled using the right sideband $5.38<M_{B}<5.75 \mathrm{GeV} / \mathrm{c}^{2}$ of the B mass distribution, where the muon and electron contributions are expected to be minimal. The sideband $d E / d x$ pull distribution is modeled as a log-normal distribution, whose parameters are fixed in the fit.

The fit returns directly a muon fraction which has a value of:

$$
f_{\mu \mu}^{d E / d x}=\frac{N_{\mu \mu}}{N_{e e}+N_{\mu \mu}}=0.919 \pm 0.017(\text { stat. }) .
$$

\footnotetext{
${ }^{5}$ The specific value of $f_{p}$ does not make any difference in the results. What fraction of this background is from protons and what fraction is from kaons is irrelevant for this analysis, just the total fraction from both protons and kaons together is needed. This is ultimately determined by $f_{K}$, which is still free.
} 


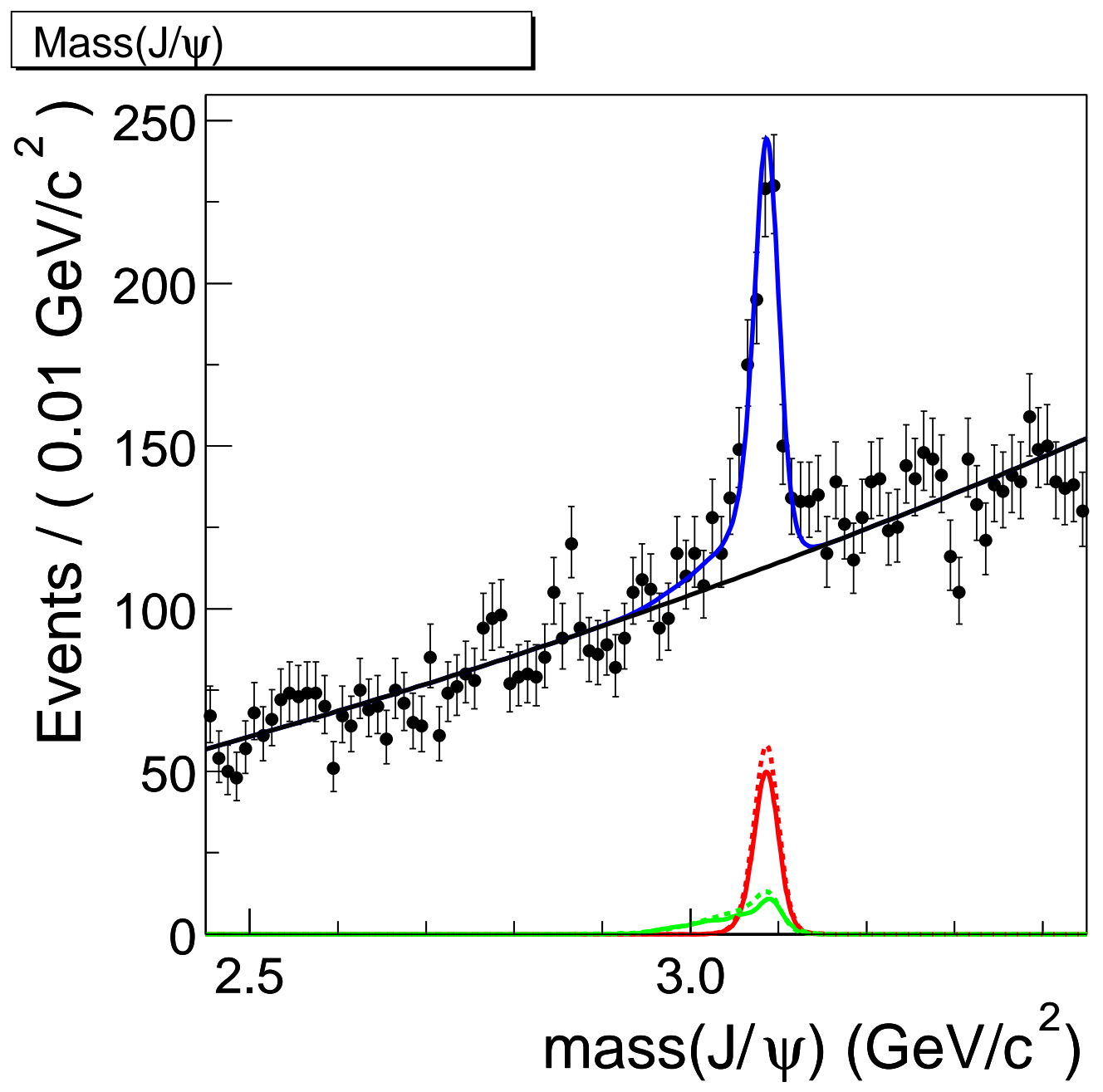

Figure 5.12: Plot of the $J / \psi$ mass in data. Red: PDF for $J / \Psi \rightarrow \mu \mu$ (two Gaussians), Green: PDF for $J / \Psi \rightarrow e e$ (a Gaussian and a bifurcated Gaussian). 


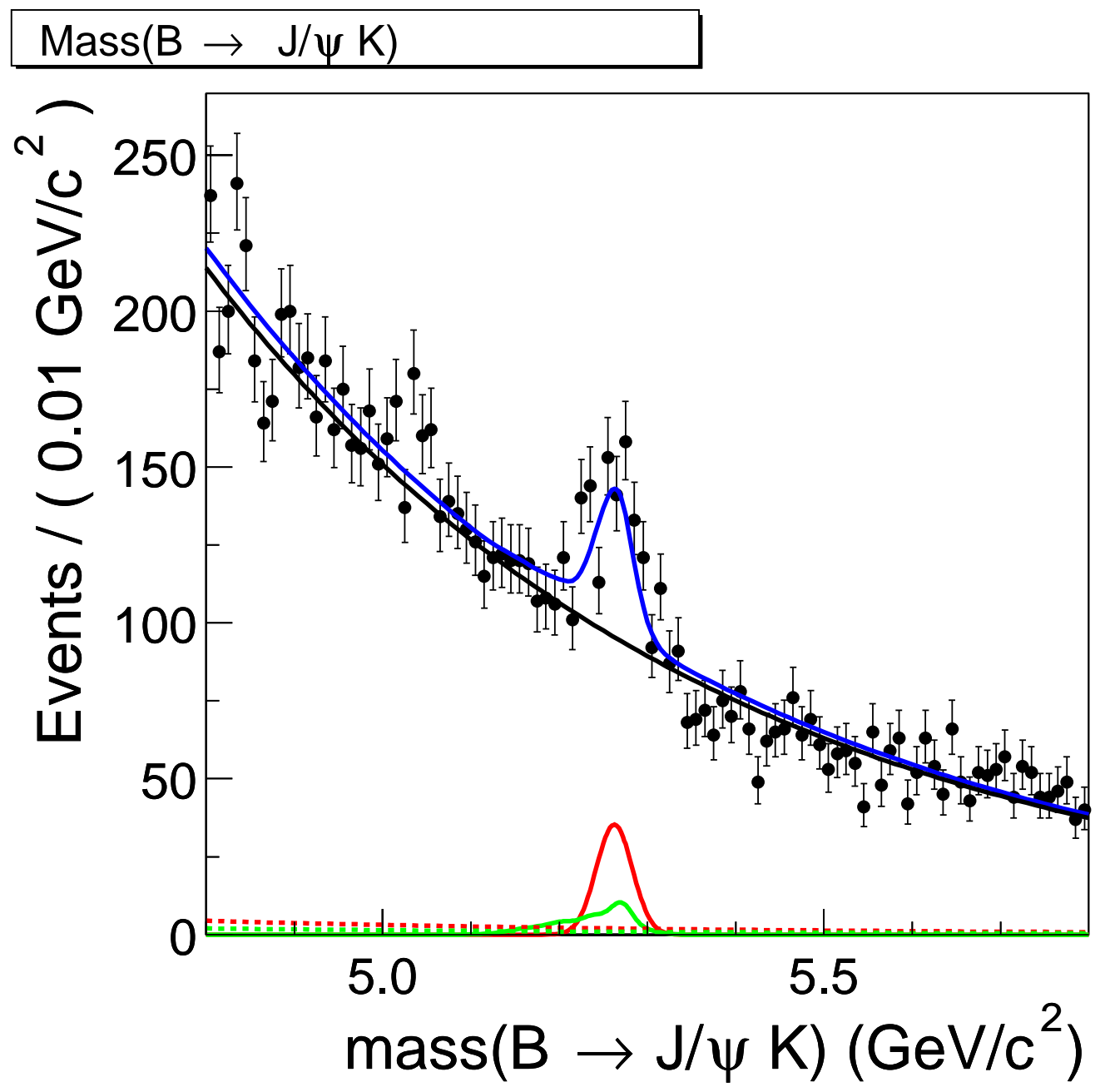

Figure 5.13: Plot of the $B$ mass in data. Red: $\mathrm{PDF}$ for $B \rightarrow J / \Psi K \rightarrow \mu \mu K$ (a Gaussian), Green: PDF for $B \rightarrow J / \Psi K \rightarrow e e K$ (1-d projection of six 2-d Gaussians) 

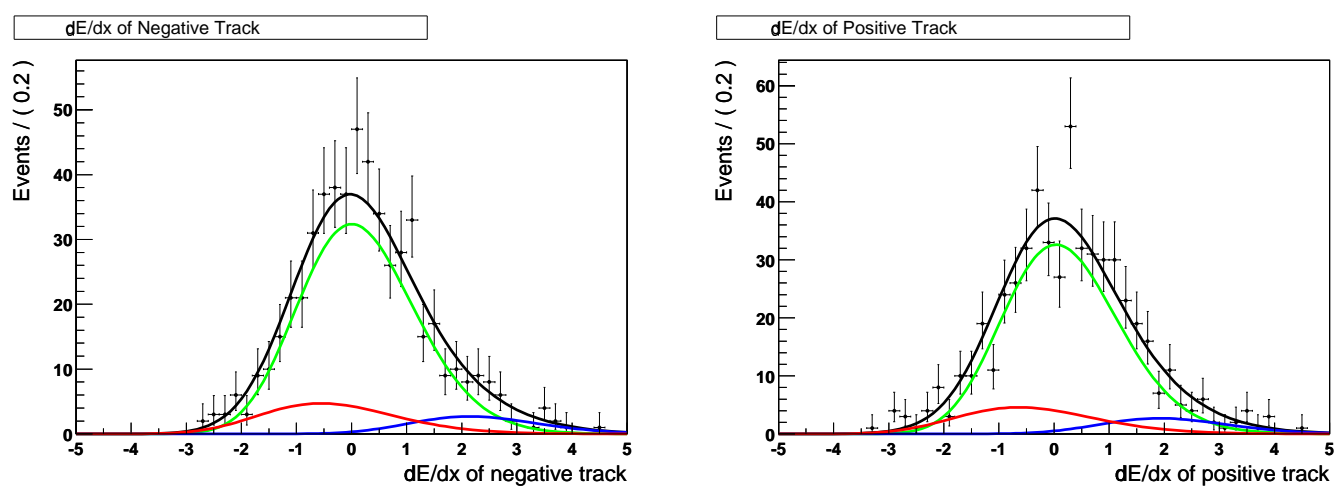

Figure 5.14: Left: $d E / d x$ pull projection for negatively charged particles. Right: $d E / d x$ pull projection for positively charged particles.

\section{$\operatorname{Mass}(\mathrm{B} \rightarrow \mathrm{J} / \psi \mathrm{K})$}

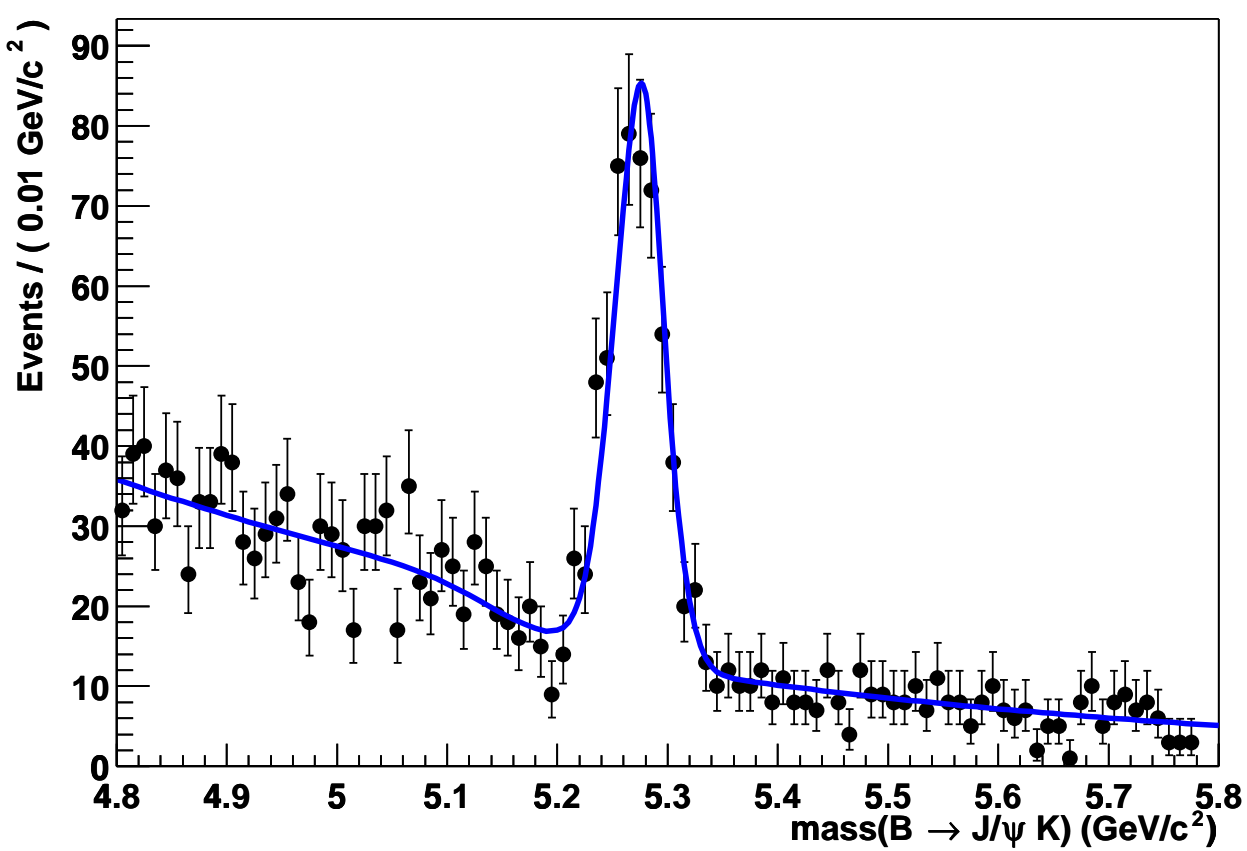

Figure 5.15: Projection of the 4-dimensional fit on the B mass dimension after applying the analysis cuts on the $J / \psi$ mass. 


\begin{tabular}{|c|c|c|c|}
\hline Fit Parameter & Value & Error & Description \\
\hline$R_{B / S}$ & 0.865 & 0.098 & $\begin{array}{l}\text { Ratio of } J / \psi \text { in combinatorial background } \\
\text { true } J / \psi \text { from signal }\end{array}$ \\
\hline$N_{b k g}$ & 18935 & 142 & \# of true combinatorial background events \\
\hline$N_{e e K}$ & 122 & 13 & $\begin{array}{l}\text { \# of events from } J / \psi \rightarrow e^{+} e^{-} \text {(including } \\
\text { combinatorial background of } B \rightarrow J / \psi K \text { ) }\end{array}$ \\
\hline$N_{\mu \mu K}$ & 385 & 24 & $\begin{array}{l}\text { \# of events from } J / \psi \rightarrow \mu \mu \text { (including } \\
\text { combinatorial background of } B \rightarrow J / \psi K \text { ) }\end{array}$ \\
\hline$\tau,\left(\mathrm{GeV} / \mathrm{c}^{2}\right)^{-1}$ & -1.717 & 0.027 & Exponential coef. for the B mass comb. bkg. \\
\hline$f_{K}$ & 0.1011 & 0.0043 & $\begin{array}{l}\text { Fraction of the kaons in true comb.bkg. } \\
\text { (Needed by dE/dx PDF). }\end{array}$ \\
\hline$f_{e}$ & 0.0597 & 0.0022 & $\begin{array}{l}\text { Fraction of the electrons in true comb.bkg. } \\
\text { (Needed by } d E / d x \text { PDF). }\end{array}$ \\
\hline$f_{\text {phys bkg }}$ & 0.71 & 0.11 & Physical background fraction \\
\hline$\mu_{J / \psi}, \mathrm{GeV} / \mathrm{c}^{2}$ & 3.09413 & 0.00091 & $J / \psi$ mass \\
\hline$\mu_{B}, \mathrm{GeV} / \mathrm{c}^{2}$ & 5.2741 & 0.0017 & B mass \\
\hline$\sigma_{B}, \mathrm{GeV} / \mathrm{c}^{2}$ & 0.0236 & 0.0014 & Width of $B \rightarrow J / \psi K$ mass peak \\
\hline $\mathrm{p} 1,\left(\mathrm{GeV} / \mathrm{c}^{2}\right)^{-1}$ & 14432 & 77 & Slope of $J / \psi$ mass comb. bkg. \\
\hline
\end{tabular}

Table 5.6: Results of fit to determine $\mu \mu$ fraction in $B \rightarrow J / \psi K$ signal using the 4-dimensional method. 


\subsubsection{Fraction of $J / \psi \rightarrow \mu^{+} \mu^{-}$From the Monte Carlo}

The realistic Monte Carlo gives good agreement with the data in $B^{ \pm} \rightarrow J / \psi K^{ \pm}$ for both the $\mu^{+} \mu^{-}$and the $e^{+} e^{-}$channels, so the relative efficiency in the two Monte Carlo channels was used to estimate the muon fraction in the data sample. From the Monte Carlo sample previously described a muon fraction of:

$$
f_{\mu \mu}^{M C}=\frac{\epsilon_{\mu \mu}}{\epsilon_{e e}+\epsilon_{\mu \mu}}=0.799 \pm 0.011 \text { (stat.) }
$$

is found, where the error is the statistical error from the Monte Carlo sample.

A source of concern is the ability of the present simulation to determine the peak position of the two signals in the $J / \psi$ mass dimension and the shape, which in the $e^{+} e^{-}$channel shows a large tail toward lower mass due to their bremsstrahlunging in the detector. Hence the need for confirmation of this value by the other methods.

\subsubsection{Fraction of $J / \psi \rightarrow \mu^{+} \mu^{-}$From Muon Chamber Identifi- cation}

As an additional cross check the muon fraction was calculated using information from the muon detectors. Define $N_{S G N}$ and $N_{C B}$ as the number of events in the signal region and the number of combinatorial background events expected in the signal region, respectively, where $N_{C B}$ is determined by counting the number of events in the sidebands and rescaling appropriately. The signal region is defined as $\left|M_{\mu \mu K}-5.28\right|<$ $0.06 \mathrm{GeV} / \mathrm{c}^{2}$, and the sidebands are given by $0.10<\left|M_{\mu \mu K}-5.28\right|<0.16 \mathrm{GeV} / \mathrm{c}^{2}$. $N_{S G N}^{\mu}$ and $N_{C B}^{\mu}$ are similarly defined with the additional requirement that at least one of the $J / \psi$ legs is associated to a CMU stub, where: $|\Delta x|<40 \mathrm{~cm}$, and $|\Delta \phi|<0.5$.

The raw muon fraction is then defined as:

$$
f_{\mu \mu}^{\mathrm{raw}}=\frac{N_{S G N}^{\mu}-N_{C B}^{\mu}}{N_{S G N}-N_{C B}}=\frac{241-22}{450-82}=0.595 \pm 0.026
$$

Fig. 5.16 shows the distributions for the muon stub-track matching variables $|\Delta x|$ and $|\Delta \phi|$ in both Monte Carlo and data. The agreement is quite good. The global 

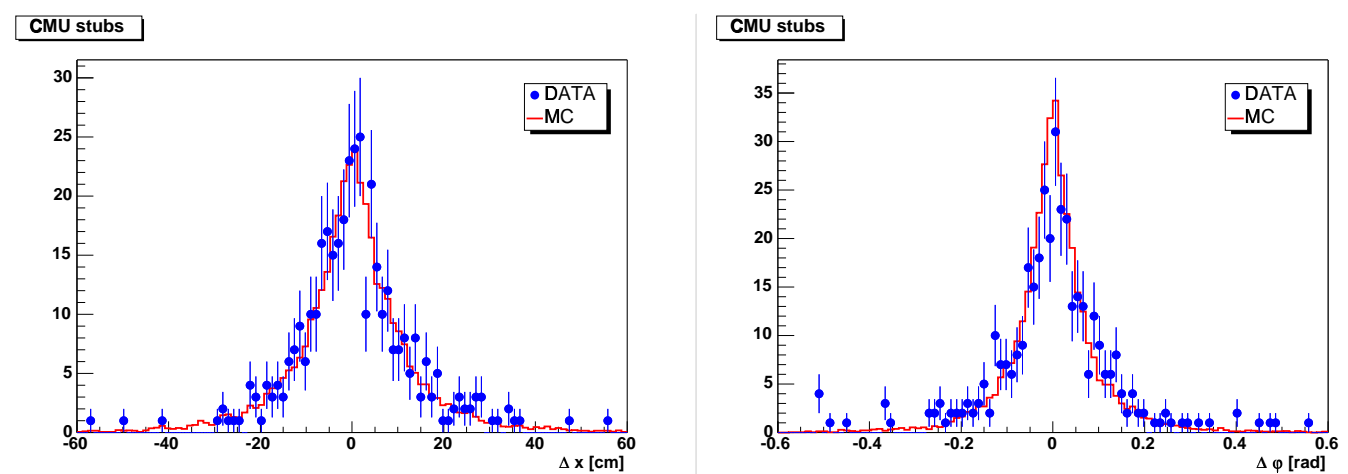

Figure 5.16: $|\Delta x|$ and $|\Delta \phi|$ distributions in data and Monte Carlo.

efficiency to associate a muon stub to a track is calculated using the realistic Monte Carlo, and is estimated to be $\varepsilon_{M C}=0.492 \pm 0.004$. This number has been corrected for the muon reconstruction efficiency of $0.986 \pm 0.003$ ([52]) and accounts for the CMU system's geometric acceptance, the stub reconstruction efficiency, and the stub-track association efficiency.

The probability to find at least one CMU stub associated to one of the two $\mathrm{J} / \psi$ legs is given by $2 \varepsilon_{M C}\left(1-\varepsilon_{M C}\right)+\varepsilon_{M C}^{2}$. The muon fraction in the signal region is therefore given by

$$
f_{\mu \mu}=\frac{f_{\mu \mu}^{\mathrm{raw}}}{2 \varepsilon_{M C}-\varepsilon_{M C}^{2}}=0.802 \pm 0.036(\text { stat. }) \pm 0.005 \text { (syst.), }
$$

where the quoted statistical error accounts for the uncertainty in the correcting coefficients.

\subsubsection{Final Result for $J / \psi \rightarrow \mu^{+} \mu^{-}$Fraction}

Table 5.7 summarizes the results for the muon fraction obtained using the methods described in the previous sections. Other than the 2-dimensional $d E / d x$ fit, they are all consistent within their associated errors. This discrepancy will be discussed further in Section 6.5, where a systematic error will be assigned to the estimated muon fraction. 


\begin{tabular}{|l|c|}
\hline \hline method & value \\
\hline \hline $4 \mathrm{D}$ fit & $0.839 \pm 0.016$ \\
$d E / d x$ fit & $0.919 \pm 0.017$ \\
Monte Carlo & $0.799 \pm 0.003$ \\
Muon Id & $0.802 \pm 0.036$ \\
\hline \hline
\end{tabular}

Table 5.7: Comparison of the estimates for the muon fraction in the $B^{ \pm} \rightarrow J / \psi K^{ \pm}$ signal region. Only statistical errors are quoted. 


\section{Chapter 6}

\section{Evaluation of Systematic Errors}

Before calculating the final ratio of branching ratios, the systematic uncertainties introduced in the measurement must be estimated. The uncertainties associated with the efficiency parameters calculated using Monte Carlo are taken directly from Tables 5.2 and 5.3. The remaining uncertainties and their estimated values are discussed in the remainder of this chapter. A summary of the results can be found in Table 6.1.

\subsection{Width Constraint on $B \rightarrow \phi K$ Fit}

Fig. 6.1 shows the $D_{s}$ meson mass distribution for events of the type $D_{s} \rightarrow \phi \pi$ for both data and Monte Carlo. It can be seen that in this mode, which is topologically similar to $B^{ \pm} \rightarrow \phi K^{ \pm}$, the Monte Carlo underestimates the experimental resolution on the mass measurement, the P2 parameter in the fits in Fig. 6.1, by approximately 20\%. From a linear+single Gaussian fit to the data a signal width of $7.59 \pm 0.06 \mathrm{MeV} / \mathrm{c}^{2}$ is obtained, which should be compared with the value of $6.17 \pm 0.06 \mathrm{MeV} / \mathrm{c}^{2}$ obtained from Monte Carlo.

Furthermore, in the sample of $B^{ \pm} \rightarrow J / \psi K^{ \pm}$decays the experimental width of the peak is found to be $24.0 \pm 0.2 \mathrm{MeV} / \mathrm{c}^{2}$ when using only events where at least one muon is identified. The Monte Carlo prediction for this peak width is $18.5 \mathrm{MeV} / \mathrm{c}^{2}$. The ratio of these two values is used to scale the $18 \mathrm{MeV} / \mathrm{c}^{2}$ peak width predicted by the Monte Carlo for the $B^{ \pm} \rightarrow \phi K^{ \pm}$channel, giving an expected peak width in the 


\begin{tabular}{lc}
\hline \hline SYSTEMATIC & ERROR \\
\hline Width constraint on $B^{ \pm} \rightarrow \phi K^{ \pm}$fit & $4.7 \%$ \\
Muon fraction in $B^{ \pm} \rightarrow J / \psi K^{ \pm}$ & $7.9 \%$ \\
Narrow $\phi$ opening angle effect on trigger & $2.0 \%$ \\
$\epsilon_{\phi K}$ uncertainty from Monte Carlo & $0.9 \%$ \\
$\epsilon_{\mu \mu K}$ uncertainty from Monte Carlo & $1.1 \%$ \\
Isolation cut relative efficiencies & $0.9 \%$ \\
$\chi_{x y}^{2}$ simulation & $3.0 \%$ \\
\hline TOTAL & $10.0 \%$ \\
\hline \hline
\end{tabular}

Table 6.1: Contributions to the relative systematic uncertainty.
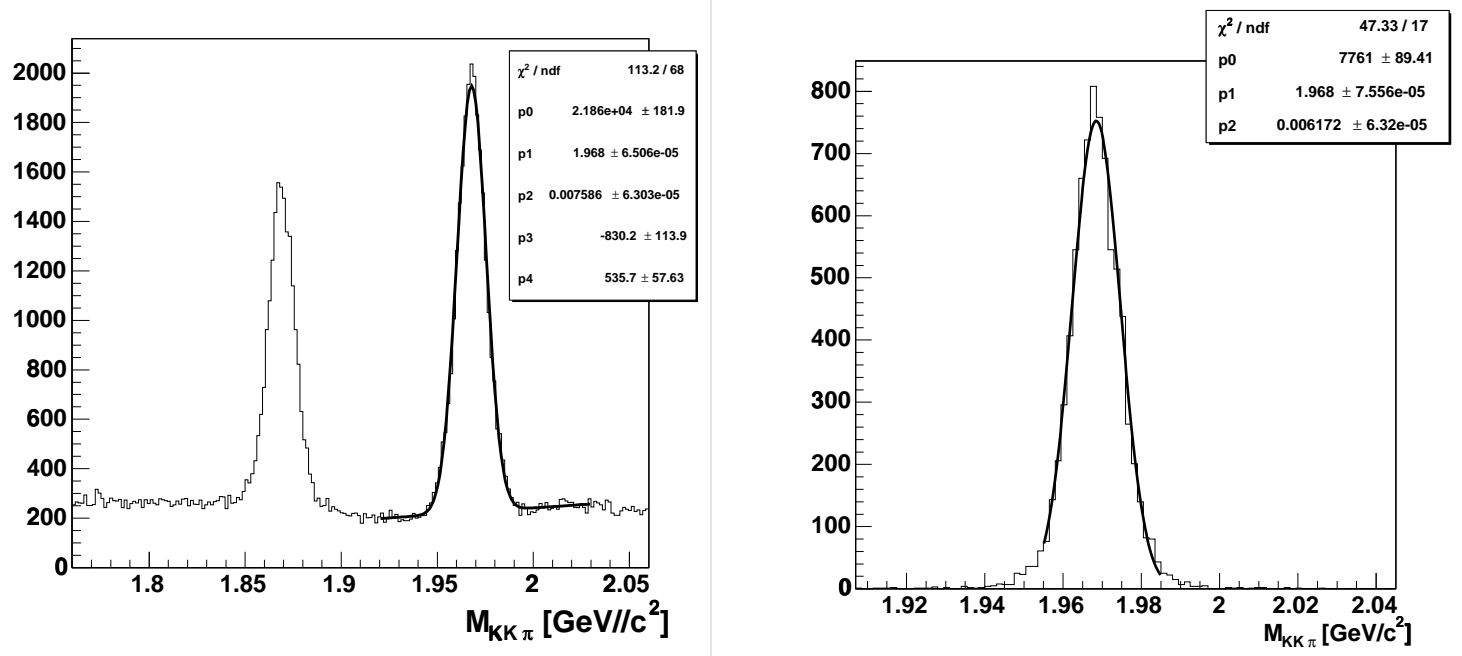

Figure 6.1: Left: $D_{s}$ and $D^{+}$mass distribution in data in the $\phi \pi$ decay mode. Right: $D_{s} \rightarrow \phi \pi$ Monte Carlo mass distribution 
data of $23 \mathrm{MeV} / \mathrm{c}^{2}$. This value was used for the width constraint applied on the fit in Section 5.2.4.

To properly account for the error introduced by the width constraint, the constraint was moved by $\pm 5 \mathrm{MeV}$-corresponding to $100 \%$ of the difference between data and Monte Carlo signal width-yielding a one event difference in either case. This corresponds to a $4.7 \%$ systematic error.

\subsection{Narrow Opening Angle of the $\phi$}

The low Q-value of the $\phi \rightarrow K K$ decay, along with the requirement that at least one of the resulting tracks have a transverse momentum greater than $2 \mathrm{GeV} / \mathrm{c}$ (i.e. be a trigger track) leads to these two tracks being highly collimated. The TTT requires an opening angle of at least 2 degrees. When the phi opening angle is less than 2 degrees only one of the phi decay tracks can participate in the trigger. The other trigger track must be from the third track (the particle that did not come from the phi decay). When the phi opening angle is greater than 2 degrees any two of the three tracks in the event can potentially participate in the trigger. The effect due to the phi tracks being collimated-on both triggering and tracking efficiency-needs to be investigated.

Fig. 6.2 shows a histogram of the number of events as a function of the $\phi$ tracks' opening angle for the decay $D_{s} \rightarrow \phi \pi$. The blue points are for the data of all events and the red points are for the data of the subset of events for which both the phi tracks participated in the trigger. Likewise, the black histogram is for the Monte Carlo data and the red histogram is for the subset of the Monte Carlo data for which both the phi tracks participated in the simulated trigger. The sharp jump in yield when the phi opening angle goes over 2 degrees can be clearly seen. The difference in the number of phi triggers between Monte Carlo and data was found to be $5.6 \%$. Since $35 \%$ of the $B^{ \pm} \rightarrow \phi K^{ \pm}$events in the realistic Monte Carlo were $\phi$ triggered this implies a $0.056 \times 0.35=2.0 \%$ systematic effect. 


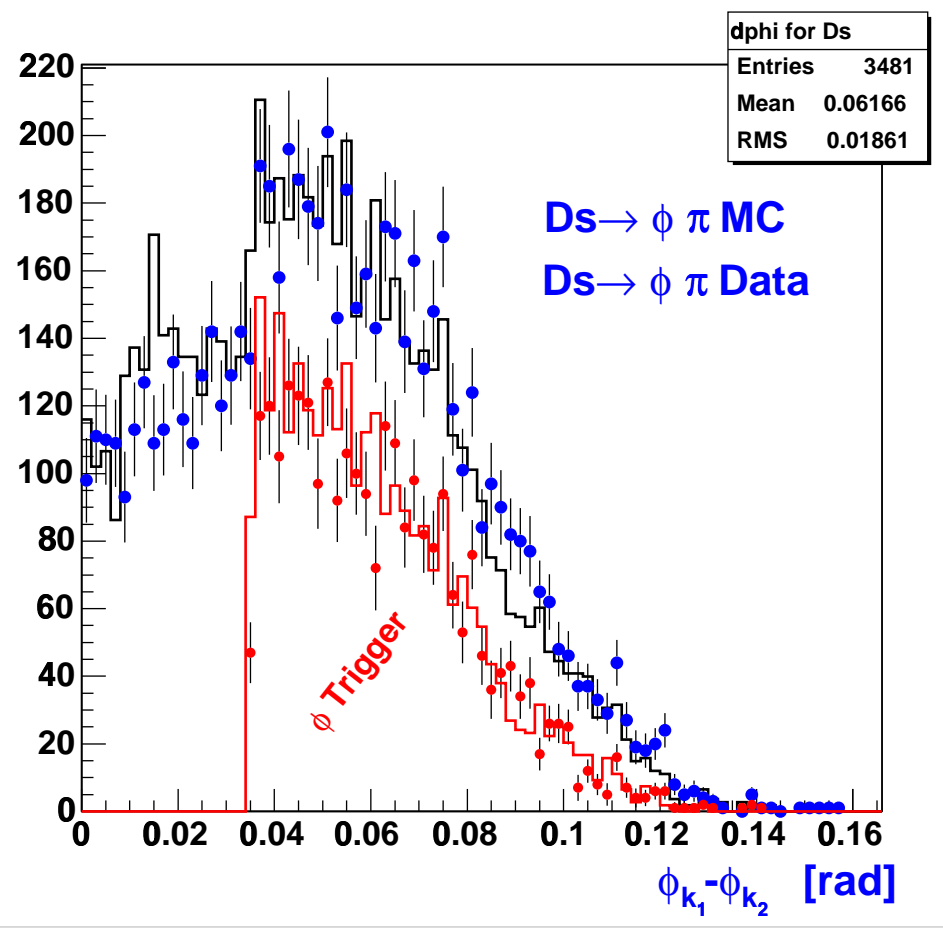

Figure 6.2: Number of events vs. opening angle of phi in $D_{s} \rightarrow \phi \pi$ events. The red points (red histogram) are for the data (Monte Carlo data) of the subset of events for which both the phi tracks participated in the trigger. MC: $2878 \phi$-triggers, Data: $2716 \phi$-triggers. 


\subsection{Isolation Cut Efficiency}

The isolation depends on the fragmentation of the initial decay particle and on its momentum. The transverse momentum spectra for the two channels are reported in Fig. 6.3, which shows that the combined trigger and selection cuts conspire to give a slightly harder spectra for the $B^{ \pm} \rightarrow J / \psi K^{ \pm}$decays. A correction must therefore be evaluated for the difference in isolation cut efficiency, which is expected to be $p_{T}$ dependent.

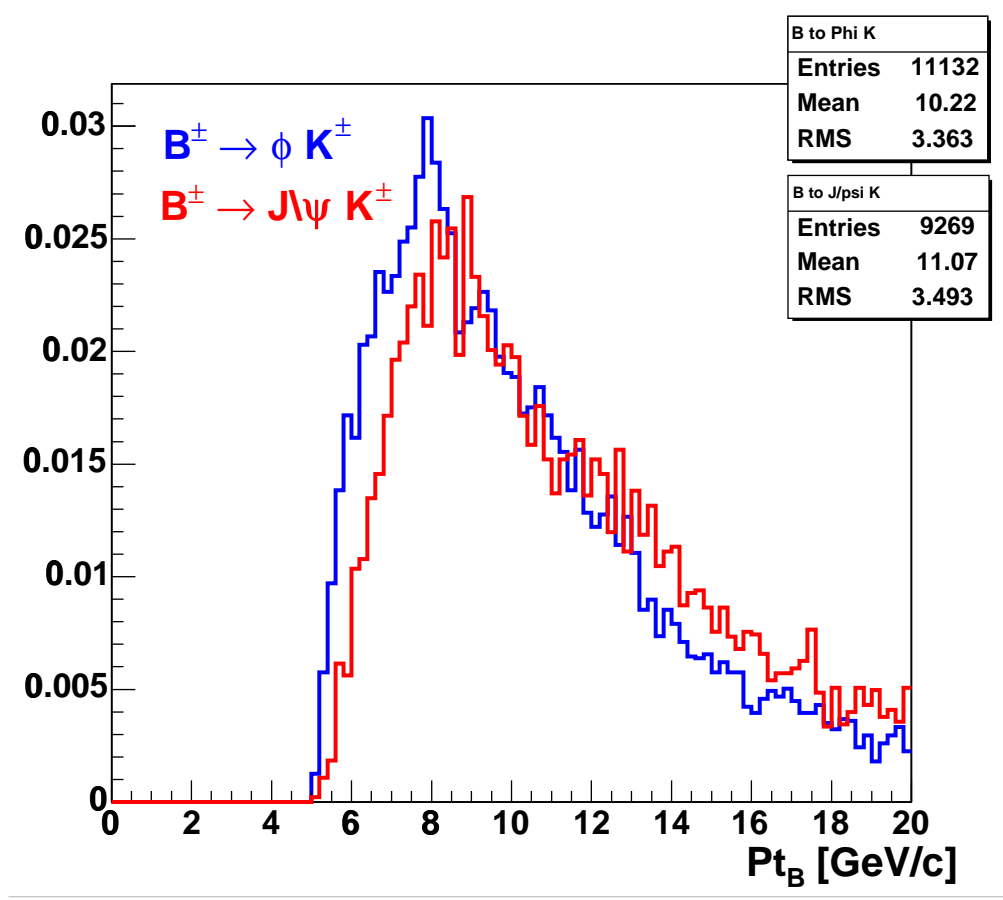

Figure 6.3: Transverse momentum spectra of the selected candidates from the Monte Carlo sample for the two decay channels.

The efficiency of the isolation cut has been evaluated on the $B^{ \pm} \rightarrow J / \psi K^{ \pm}$control channel for five intervals of $p_{T}$, as shown in Fig. 6.4, where a linear fit was applied. The parameters of the linear fit have been used to re-weight the $p_{T}$ spectra in Monte Carlo for both channels. The efficiency of the isolation cut has been evaluated for each channel as the ratio between the integrals of the weighted and un-weighted spectra. The systematic error on this measurement was derived from the statistical error on 
the linear fit parameters to $\epsilon_{i s o}\left(p_{T}\right)$. Using the range of variation shown in Fig. 6.4, the ratio of efficiencies changes by $[+5,-12] \times 10^{-3}$. This error has been symmetrized in Table 6.2. The ratio of isolation efficiencies for the cut value used in this analysis (an isolation of 0.5 ) is

$$
R\left(\epsilon_{\text {iso }}\right)=1.028 \pm 0.009
$$

As expected due to the similarity of the two decays, the isolation efficiency correction is small.

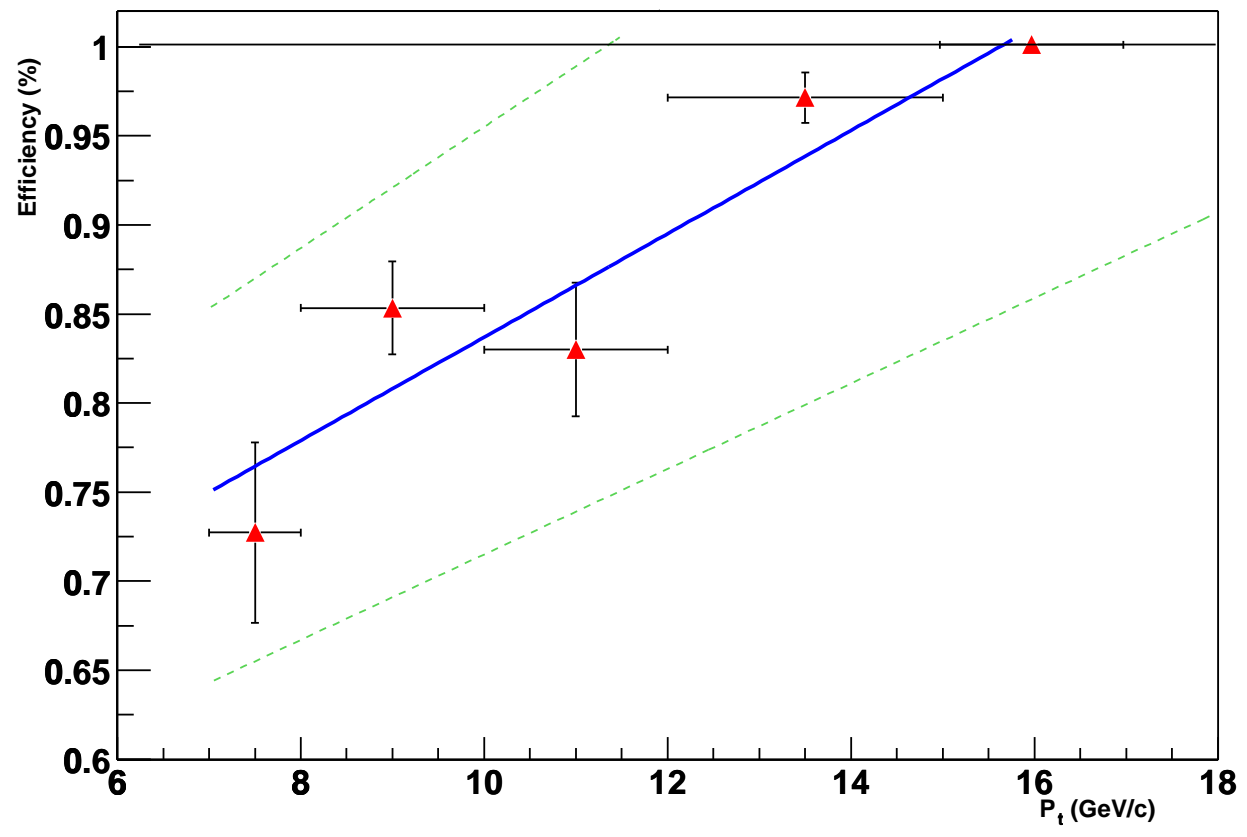

Figure 6.4: Efficiency of the isolation cut at 0.5, as measured with the $B^{ \pm} \rightarrow J / \psi K^{ \pm}$ channel. The dashed lines represent the statistical variation in the linear fit and are one standard deviation to either side.

\section{4 $\chi_{x y}^{2}$ Cut Efficiency}

In the final calculated results, the $\chi_{x y}^{2}$ efficiency is treated as being the same for the two channels. This is not necessarily a good approximation, given the slight 


\begin{tabular}{|l|l|}
\hline Isolation efficiency intercept & $(0.545 \pm 0.07)$ \\
Isolation efficiency slope & $(0.029 \pm 0.005)\left(\mathrm{GeV}^{-1}\right)$ \\
Efficiency for $\phi K$ & $(85.3 \pm 0.3) \%$ \\
Efficiency for $J / \psi K$ & $(87.7 \pm 0.3) \%$ \\
\hline Ratio of efficiencies & $1.028 \pm 0.009$ \\
\hline
\end{tabular}

Table 6.2: Parameters of the linear fit to the isolation efficiency as a function of $B^{ \pm}$ meson $p_{T}$.

differences in the two vertex topologies. Additionally, the Monte Carlo does not reproduce the efficiency of this cut particularly well. This can be seen in Fig. 6.5 which compares the $\chi_{x y}^{2}$ cut efficiency in Monte Carlo and data for the $D_{s} \rightarrow \phi \pi$ and $B^{ \pm} \rightarrow J / \psi K^{ \pm}$channels.

To bound the associated systematic error, the double ratio of efficiencies was calculated for data and Monte Carlo in the $D_{s} \rightarrow \phi \pi$ and $B^{ \pm} \rightarrow J / \psi K^{ \pm}$channels. Fig. 6.5 shows the efficiency in data and Monte Carlo as a function of the $\chi_{x y}^{2}$ cut. The ratio of the two efficiency ratios between the two channels for the cut value used in the analysis $\left(\chi_{x y}^{2}<20\right)$ is $1.031 \pm 0.015$. As a systematic, $100 \%$ of the effect measured on the ratio of $D_{s} \rightarrow \phi \pi$ to $B^{ \pm} \rightarrow J / \psi K^{ \pm}$events is used. This is conservative, since the $B^{ \pm} \rightarrow \phi K^{ \pm}$vertices should be more similar to the $B^{ \pm} \rightarrow J / \psi K^{ \pm}$ones than those of the $D_{s}$.

\subsection{Estimated Muon Fraction in $B^{ \pm} \rightarrow J / \psi K^{ \pm}$}

The systematics associated with the fit to determine the muon fraction in $B^{ \pm} \rightarrow$ $J / \psi K^{ \pm}$come from two sources. First, the $d E / d x$ calibrations are not yet final and large variation in the $d E / d x$ responses are reported for different classes of tracks, in particular between the tracks that are seen by the XFT (and thus are able to participate in trigger decisions) and the generic tracks. The second systematic effect 

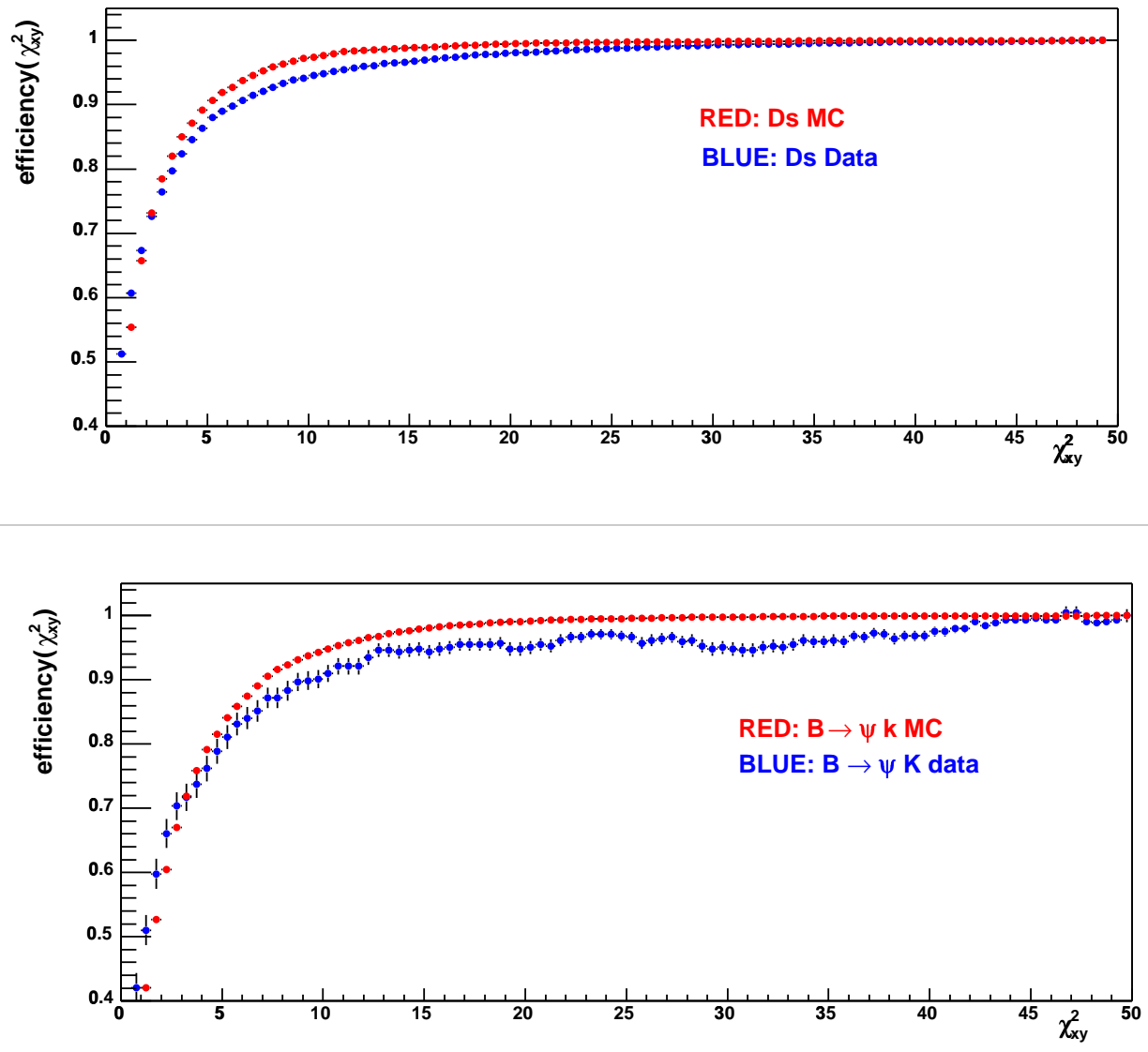

Figure 6.5: Efficiency of the $\chi_{x y}^{2}$ cut as measured for $D_{s} \rightarrow \phi \pi$ (above) and $B^{ \pm} \rightarrow$ $J / \psi K^{ \pm}$(below) compared to the same quantity in the corresponding Monte Carlo sample. 


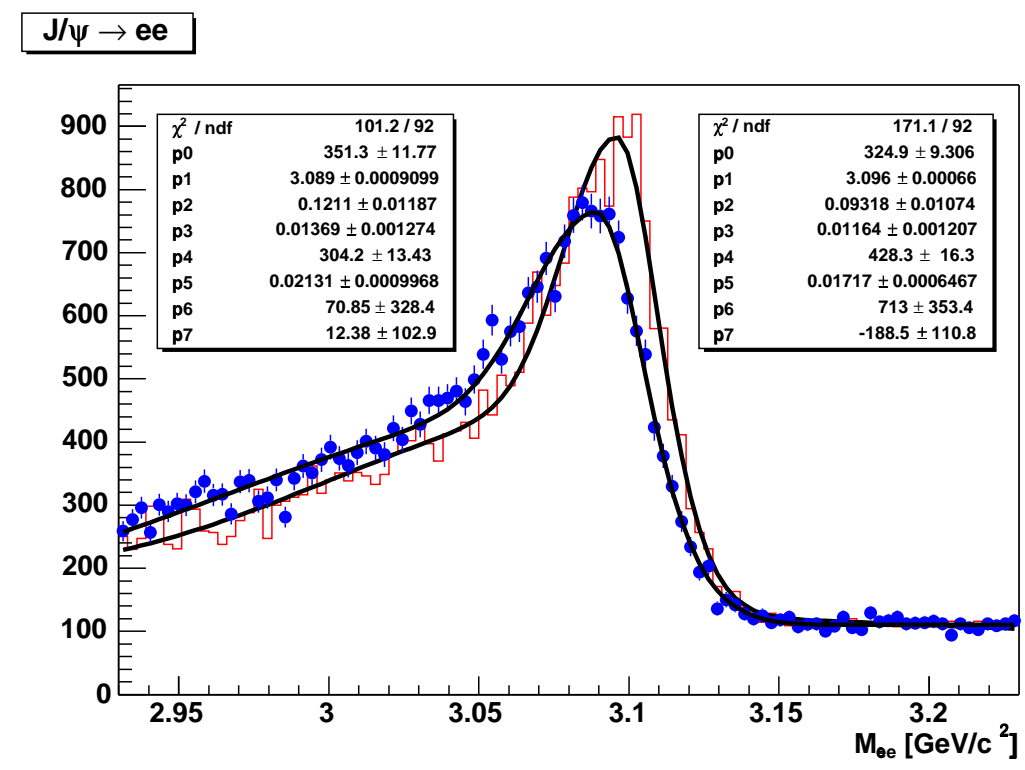

Figure 6.6: Plot of the $J / \psi$ mass in data and Monte Carlo for the electron channel.

is associated to the uncertainty in the modeling of the electron bremsstrahlung, which affects the mass shape for the $e^{+} e^{-}$channel.

To evaluate the $d E / d x$ systematics the sample was first divided into two periods, corresponding to data before and after the January 2003 shutdown. There was no significant change in the fit results between these two time spans. The sample was also divided into the two cases where one or both of the $J / \psi$ legs participated in the trigger. In this case significant changes in the fit results are seen. When both $J / \psi$ legs trigger the event a muon fraction of $f_{\mu \mu}=0.861 \pm 0.018$ is measured in the fit, whereas a muon fraction of $f_{\mu \mu}=0.781 \pm 0.033$ is measured in the fit when only one leg is part of the trigger decision. A systematic error is therefore assigned corresponding to the maximum variation with respect to the default value, rescaled to the fraction of times that only one leg of the $J / \psi$ is part of the trigger decision $(30 \%)$.

For the second source of systematics, the same $J / \psi \rightarrow e^{+} e^{-}$data used for the $d E / d x$ calibration was compared to the $B^{ \pm} \rightarrow J / \psi K^{ \pm}$Monte Carlo. Fig. 6.6 shows the Monte Carlo and data samples for $J / \psi \rightarrow e^{+} e^{-}$. Applying the same cuts as used 
in the analysis, it is seen that the Monte Carlo overestimates the efficiency by about $10 \%$. To evaluate the systematic on the muon fraction determination, the number of electron events returned by the fit was scaled by the same amount.

The following result for the complete 4-dimensional fit is therefore given:

$$
f_{\mu \mu}^{f i t}=0.839 \pm 0.016(\text { stat. }) \pm 0.028(\text { syst. })
$$

Finally, recall that the muon fraction obtained in the $d E / d x$-only fit-one of the three cross-checks done for this value-was not consistent with the results of the other methods (see Table 5.7). While there might well be some additional systematics related to the $d E / d x$ that could explain this discrepancy, in order to be conservative the full spread of these values is taken into account by adding a $7 \%$ relative systematic uncertainty to the value obtained from the 4-dimensional fit. This dominates the statistical uncertainty associated with the measurement. The value that will be used in the final calculation for the ratio of branching ratios will therefore be:

$$
f_{\mu \mu}=0.839 \pm 0.066(\text { syst.) }
$$




\section{Chapter 7}

\section{Summary and Conclusions}

As seen in Fig. 5.4, $23 \pm 7 B \rightarrow \phi K$ events are observed using $120 \pm 7 \mathrm{pb}^{-1}$ of data taken at CDF. The same cuts were used to select the decay $B^{ \pm} \rightarrow J / \psi K^{ \pm}$, yielding $406 \pm 26$ events as shown in Fig. 5.5. The ratio of branching ratios is given by the formula:

$$
\frac{B R(B \rightarrow \phi K)}{B R(B \rightarrow \psi K)}=\frac{N_{\phi K}}{N_{\psi K} \cdot f_{\mu \mu}} \frac{B R(\psi \rightarrow \mu \mu) \epsilon_{\mu \mu K}}{B R(\phi \rightarrow K K) \epsilon_{K K K}} R\left(\epsilon_{\text {iso }}\right)
$$

where

$$
N_{\phi K}=23 \pm 7(\text { stat })
$$

is the number of observed $B^{ \pm} \rightarrow \phi K^{ \pm}$events, and

$$
N_{\psi K} \cdot f_{\mu \mu}=(406 \pm 26(\text { stat })) \cdot(0.839 \pm 0.066)
$$

is the number of observed $B^{ \pm} \rightarrow J / \psi K^{ \pm}$events with $J / \psi \rightarrow \mu^{+} \mu^{-}$.

$$
\begin{aligned}
\epsilon_{\mu \mu K} & =0.00453 \pm 0.00005 \\
\epsilon_{K K K} & =0.00554 \pm 0.00005
\end{aligned}
$$

are the reconstruction efficiencies from Monte Carlo for $B^{ \pm} \rightarrow J / \psi K^{ \pm}$and $B^{ \pm} \rightarrow$ $\phi K^{ \pm}$(found in Tables 5.2 and 5.3), respectively, and

$$
R\left(\epsilon_{\text {iso }}\right)=1.028 \pm 0.009
$$

is the ratio of isolation efficiencies calculated in Section 6.3. 
The 2002 Particle Data Group[54] world average values were used for the branching fractions of $\phi \rightarrow K K$ and $J / \psi \rightarrow \mu \mu$.

$$
\begin{aligned}
B R(\phi \rightarrow K K) & =(49.2 \pm 0.7) \% \\
B R\left(J / \psi \rightarrow \mu^{+} \mu^{-}\right) & =(5.88 \pm 0.10) \%
\end{aligned}
$$

Putting all this together, the ratio of branching ratios is found to be

$$
\begin{aligned}
\frac{B R(B \rightarrow \phi K)}{B R(B \rightarrow J / \psi K)}= & \frac{(23 \pm 7)}{(410 \pm 23) \cdot(0.839 \pm 0.066)} \times \\
& \frac{(5.88 \pm 0.10) \% \cdot(0.00453 \pm 0.00005)}{(49.2 \pm 0.7) \% \cdot(0.00554 \pm 0.00005)} \times 1.028 \pm 0.009 \\
= & 0.0068 \pm 0.0021 \text { (stat.) } \pm 0.0007 \text { (syst.) }
\end{aligned}
$$

The contributions to the systematic uncertainty are listed in Table 6.1. Using this measurement and the 2002 Particle Data Group[54] value for the $B R\left(B^{ \pm} \rightarrow J / \psi K^{ \pm}\right)=$ $(1.01 \pm 0.05) \times 10^{-3}$ the $B R\left(B^{ \pm} \rightarrow \phi K^{ \pm}\right)$is calculated to be:

$$
B R\left(B^{ \pm} \rightarrow \phi K^{ \pm}\right)=(6.9 \pm 2.1(\text { stat. }) \pm 0.8(\text { syst. })) \times 10^{-6}
$$

Fig. 7.1 shows the values of $B R\left(B^{ \pm} \rightarrow \phi K^{ \pm}\right)$measured by other experiments. This result is consistent with the world average.

As CDF acquires more data, the methods used in this analysis could be used to measure separately the yields for the two decays $B^{+} \rightarrow \phi K^{+}$and $B^{-} \rightarrow \phi K^{-}$, and a charge asymmetry [described by equation (2.12)] measurement could be made. This result could be used to confirm a similar measurement recently reported by the BaBar collaboration $\mathcal{A}_{\mathcal{C} P}\left(B^{ \pm} \rightarrow \phi K^{ \pm}\right)=0.054 \pm 0.056$ (stat.) \pm 0.012 (syst.), which is consistent with the Standard Model prediction of zero[16]. Further, if CDF matches its target integrated luminosity goal of $8 \mathrm{fb}^{-1}$ by 2009, the expected number of $B^{ \pm} \rightarrow \phi K^{ \pm}$events measured will be approximately four times that used in the BaBar analysis, improving the statistical error on the measurement by roughly a factor of two. The greater statistics should also enable CDF to reduce the systematic error bar on this measurement. Further, measuring this production cross section at 


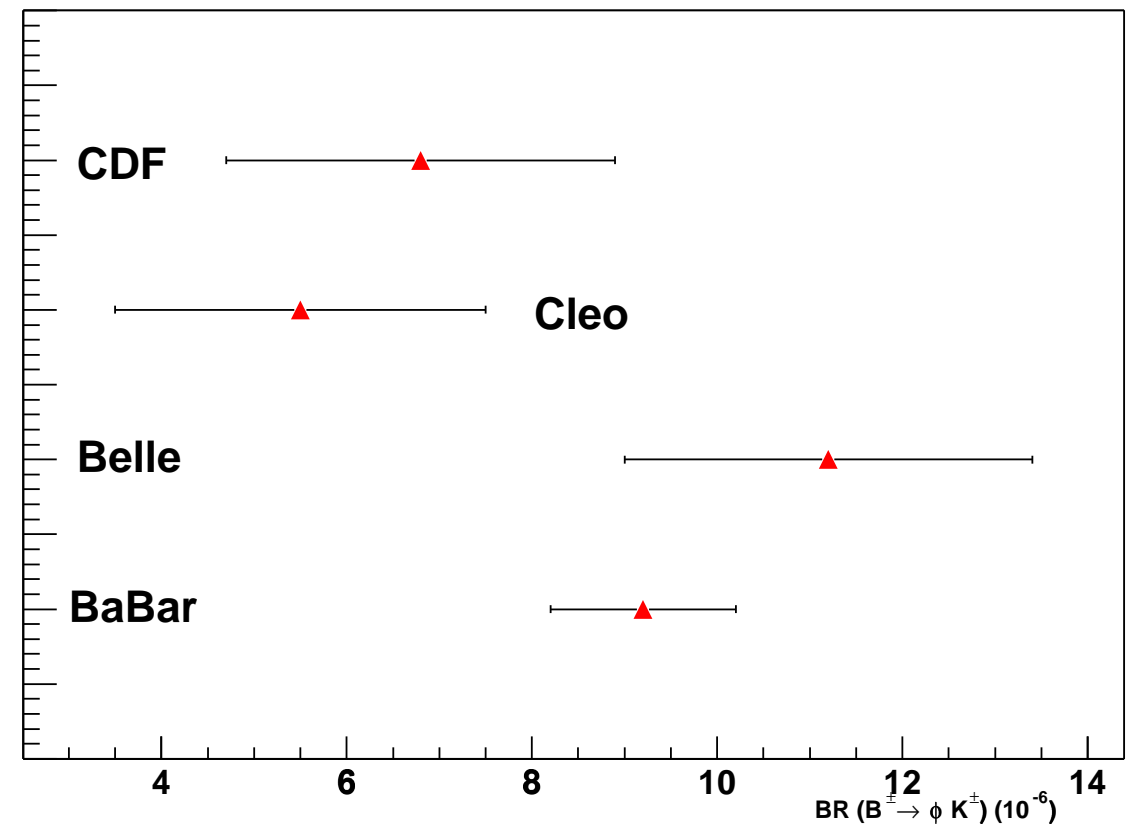

Figure 7.1: Comparison of $B R\left(B^{ \pm} \rightarrow \phi K^{ \pm}\right)$calculated using the measured value of $B R\left(B^{ \pm} \rightarrow J / \psi K^{ \pm}\right) / B R\left(B^{ \pm} \rightarrow \phi K^{ \pm}\right)$with the values reported by BaBar[1], Belle[2], and CLEO $[3]$. 
a hadron collider (Belle, Babar and CLEO are all lepton colliders) will help theorists working on better understanding and calculating QCD. With this measurement one more piece of the puzzle in our understanding of CP violation and the Standard Model will have been filled in. 


\section{Bibliography}

[1] B. Aubert et al. (BaBar Collaboration), Branching Fractions in $B \rightarrow \phi$ and Search for Direct CP Violation in $B^{ \pm} \rightarrow \phi K^{ \pm}$, BaBar-CONF-03/11 SLACPUB-9684, March 2003.

[2] K.Abe et al. (Belle Collaboration), Observation of Charmless Decays $B \rightarrow \phi K$ and $B \rightarrow \phi K^{*}$ at Belle, BELLE-CONF-0113 (2001).

[3] R.A. Briere et al. (CLEO Collaboration), Observation of $B \rightarrow$ phiK and $B \rightarrow$ phiK*, Phys.Rev.Lett. 86, 3718(2001).

[4] Perkins, Donald H., Introduction to High Energy Physics 4th ed., Cambridge University Press, 2000.

[5] Griffiths, David, Introduction to Elementary Particles, John Wiley \& Sons, Inc., 1987.

[6] H1 Collaboration, to be submitted to Phys. Lett. B [hep-ex/030403017], March 2004.

[7] Peskin, Michael E., Schroeder, Daniel V., An Introduction to Quantum Field Theory, Addison-Wesley Publishing Company, 1995.

[8] Halzen, Francis and Martin, Alan D., Introduction to Elementary Particles, John Wiley \& Sons, Inc. 1987.

[9] Deshpande, N.G., Theory of Penguins in B Decays, B Decays-Revised 2nd Ed. edited by Sheldon Stone, p. 587, World Scientific, Singapore, 1994. 
[10] Nir, Yosef, Theory of CP Violation in B Decays, B Decays-Revised 2nd Ed. edited by Sheldon Stone, p. 521, World Scientific, Singapore, 1994.

[11] A.Abashion et al. (Belle Collaboration), Measurement of the CP Violation Parameter Sin(2 $\left.\phi_{1}\right)$ in $B_{d}^{0}$ Meson Decays, Phys. Rev. Lett. 86, pp. 2509-2514, 2001.

[12] B. Aubert et al. (BaBar Collaboration), Measurement of CP-violating Asymmetries in $B^{0}$ decays to CP Eigenstates, Phys. Rev. Lett. 86, pp. 2515-2522, 2001.

[13] The Belle Collaboration, Charmless Hadronic Two-Body B Meson Decays, [hepex/0207090v1], 28 Jul. 2002.

[14] The BaBar Collaboration, Measurement of Branching Fractions and CPviolating Asymmetries in $B^{0} \rightarrow \pi^{+} \pi^{-}, K^{+} \pi^{-}, K^{+} K^{-}$Decays, Phys. Rev. Lett. 89, 281802 (2002).

[15] M.Ciuchini, L.Silvestrini, Direct CP Violation in $B \rightarrow \phi K_{s}$ and New Physics, Phys. Rev. Lett. 89, 231802, Aug 2002.

[16] B. Aubert et al. (BaBar Collaboration), Measurements of CP Asymmetries in the Decay B to Phi K, BABAR-CONF-04/33, SLAC-PUB-10603 (2004).

[17] The CDF II Collaboration, The CDF II Detector Technical Design Report, FERMILAB-Pub-96/390-E CDF, Fermi National Accelerator Laboratory, Batavia Illinois, Nov. 1996.

[18] Wenzel, Hans, Tracking in the SVX, CDF Note 1790, May 1998.

[19] Alan Sill for the CDF Collaboration, CDF Run II silicon tracking projects, Nuclear Instruments \& Methods in Physics Research Sec. A, Elsevier Science, 2000.

[20] T. Affolder et. al., CDF Central Outer Tracker, CDF Note 6267, 2003. 
[21] Guillelmo Gomez-Ceballos, et. al., Event Builder and Level 3 at the CDF Experiment, Proceedings of 9th Pisa Meeting on Advanced Detectors: Frontier Detectors for Frontier Physics, Isola d'Elba, Italy, May 25-31, 2003.

[22] J. Antos, M. Babik et al., The CDF Run2 Offline Computer Farms, CDF Note $5702,2001$.

[23] J. Lewis, D. Saltzberg and M. Shochet et. al., CDF Run-II Trigger Table and Datasets Plan, CDF Note 4718, 1998.

[24] M. Paulini and B. Wicklund et. al., Summary of Proposals for B Physics Triggers in Run II, CDF Note 5483, 2000.

[25] R.G.C. Oldeman for the CDF collaboration, Performance of CDF for B physics, Workshop on the CKM Unitarity Triangle, IPPP Durham, April 2003.

[26] A. Belloni, I.K. Furic and Ch. Paus, Multibody Trigger Paths in the Two Track Trigger Data, CDF Note 6526, 2003.

[27] http://www-cdf.fnal.gov/internal/physics/bottom/validation/

[28] W. Badgett, /emphThe CDF Run II Run Database and Online Java API, CDF Note 5672, 2001.

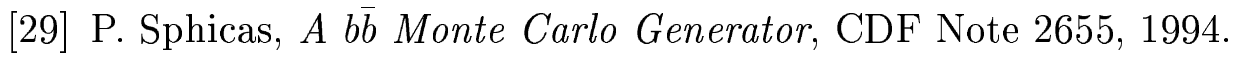

[30] K. Anikeev, C. Paus, and P. Murat, Description of Bgenerator II: Bgenerator in its Run II Incarnation, CDF Note 5092, 1999.

[31] P. Nason, S. Dawson, and R.K. Ellis, "The Total Cross-Section for the Production of Heavy Quarks in Hadronic Collisions", Nucl. Phys. B303:607, 1988;

[32] P. Nason, S. Dawson, and R.K. Ellis, "The One Particle Inclusive Differential Cross-Section for Heavy Quark Production in Hadronic Collisions", Nucl. Phys. B327:49, 1988. 
[33] A.D. Martin, R.G. Roberts and W.J. Stirling, "Parton Distributions of the Proton", Phys.Rev. D50 (1994) 6734-6752.

[34] MRS Parton Distribution webpage, http://durpdg.dur.ac.uk/hepdata/mrs.html

[35] J.Lewis, P.Avery, CLEOMC: The CDF Interface to the CLEO Monte Carlo (QQ), CDF Note 2724, 1994

[36] The CLEO Collaboration, http://www.lns.cornell.edu/public/CLEO/

[37] S. Agostinelli, et al., [GEANT4 Collaboration] "GEANT4A Simulation Toolkit", Nucl. Instr. and Meth. A506 (2003), 250-303.

[38] R. Brun and F. Carminati, GEANT Detector Description and Simulation Tool, CERN Program Library Long Write-up W5013; see also: http://wwwasd.web.cern.ch/wwwasd/geant/

[39] A.Mukherjee, CTC and VTX Tracking, CDF Note 5490, 2000.

[40] C. Hays, et al., The COT Patter Recognition Algorithm and Offline Code, CDF Note 6992, April 2004.

[41] P.Azzi, G.Busetto et al., Histogram Tracking in the COT, CDF Note 5562, 2001.

[42] W. Yao and K. Bloom, Outside-In Silicon Tracking at CDF, CDF Note 5991, 2002.

[43] M.Campanelli, E.Gerchtein, Calibration of the momentum scale for Kalman refitter using $J / \psi$ events., CDF Note 6905, Mar. 2004.

[44] S.Menzemer, TrackingKal-A Tracking and Alignment Software Package for the CDFII Silicon Detector, CDF Note 5968 Rev.4, Feb. 2003.

[45] G.Bauer, A.Korn, Ch.Paus, Update on Calibration of Energy Loss and Magnetic Field using $J / \psi$ Events in Run II, CDF Note 6355, Mar. 2003.

[46] http://cdfkits.fnal.gov/CdfCode/source/svtsim/src/ 
[47] A.Cerri, Comparing SVT and offline tracking in the quest for SVT efficiency and over-efficiency, CDF Note 4710, Aug. 1998.

[48] S.D'Auria, et al., The CharmMods/DFinder Reconstruction Package, CDF Note 6158, Mar. 2004.

[49] J.Boudreau, P.Maksimovic, The Universal Finder, CDF Note 5217, Jan. 2000.

[50] S.D'Auria, et al., dE/dx Performance Studies Using $D^{* \pm}$, CDF Note 6369, Mar. 2003.

[51] J.Marriner, Secondary Vertex Fit, CDF Note 1996, Jun. 1991.

[52] K. Bloom and W. D. Dagenhart, Muon-Reconstruction Efficiency for Winter 2003 Conferences, CDF Note 6347.

[53] H.Nelson, T.Nelson, A Guide to Unbinned Maximum Likelihood Fits, CBX 9861, Oct. 1998.

[54] Particle Physics Booklet, Particle Data Group, Lawrence Berkley National Laboratory, 2002.

[55] S. Torre et al., $d E / d x$ studies with protons from $\Lambda$ decays, Reco Tag Meeting, June 20, 2003.

[56] A.Korn, Ch.Paus, A J/Psi to mu mu Trigger Study for Run II, CDF Note 5093, Aug. 1999.

[57] M.Tanaka, J/psi to ee Trigger for CDF Run II, CDF Note 5189, Nov. 1999. 


\section{Vita}

Robert Napora was born in Elgin, Illinois, on November 29th, 1971. He attended Minot High School in North Dakota and graduated in 1989. He subsequently enlisted in the United States Marine Corps, attending boot camp in San Diego, California. He then attended the Computer Sciences School in Quantico, Virginia where he underwent training as a Computer Programmer and as a Small Computer Systems Specialist. After his technical training he was stationed overseas for two years in Okinawa, Japan, during which time he attended the Non-Commissioned Officer School. He finished out his enlistment at Headquarters Marine Corps in Washington, D.C. During his time in the Marine Corps he was promoted through to the rank of Sergeant, and was awarded both the Navy Achievement Medal and the Navy Commendation Medal.

After his tour in the Marines, he enrolled as an undergraduate at North Dakota State University where he received his Bachelor of Science degree with majors in Physics and Mathematics and a minor in Chemistry. During that time he did research in Theoretical Chemistry for the Chemistry Department, and worked as a teaching assistant for both the Physics and the Mathematics Departments.

He then attended The Johns Hopkins University as a graduate student in Physics. He worked as both a teaching assistant and head teaching assistant before moving to Illinois to conduct his research at the Collider Detector at Fermilab. He earned his Master of Arts degree in Physics in 2002.

Most recently, he has been teaching as a Visiting Assistant Professor of Physics at North Park University in Chicago, Illinois. 